\title{
Introduction to Appendices
}

Five papers of which I am co-author are included as appendices to my thesis. Here I will briefly describe my contribution to each of the papers.

For "Ferromagnetic resonance spectroscopy for assessment of magnetic anisotropy and magnetostatic interactions: A case study of mutant magnetotactic bacteria" and "Chains, clumps, and strings: Magnetofossil taphonomy with ferromagnetic resonance spectroscopy” I carried out the mutagenesis described in Chapter 2 of this thesis.

For "The paleoproterozoic snowball Earth: A climate disaster triggered by the evolution of oxygenic photosynthesis" I was involved in the discussion and development of the manuscript, particularly the biochemical arguments.

For "Experimental observation of magnetosome chain collapse in magnetotactic bacteria: Sedimentological, paleomagnetic, and evolutionary implications" I was involved in the discussion and development of the manuscript.

For "Bugbuster — survivability of living bacteria upon shock compression” I assisted with the microscopic examination of the samples, development of the project, and discussion of the manuscript. 


\title{
Ferromagnetic resonance spectroscopy for assessment of magnetic anisotropy and magnetostatic interactions: A case study of mutant magnetotactic bacteria
}

\author{
Robert E. Kopp, ${ }^{1}$ Cody Z. Nash, ${ }^{1}$ Atsuko Kobayashi, ${ }^{2}$ Benjamin P. Weiss, ${ }^{3}$ \\ Dennis A. Bazylinski, ${ }^{4}$ and Joseph L. Kirschvink ${ }^{1}$ \\ Received 25 May 2006; revised 28 September 2006; accepted 25 October 2006; published 28 December 2006.
}

[1] Ferromagnetic resonance spectroscopy (FMR) can be used to measure the effective magnetic field within a sample, including the contributions of both magnetic anisotropy and magnetostatic interactions. One particular use is in the detection of magnetite produced by magnetotactic bacteria. These bacteria produce single-domain particles with narrow size and shape distributions that are often elongated and generally arranged in chains. All of these features are detectable through FMR. Here, we examine their effects on the FMR spectra of magnetotactic bacteria strains MV-1 (which produces chains of elongate magnetite crystals), AMB-1 (which produces chains of nearly equidimensional magnetite crystals), and two novel mutants of AMB-1: mnm13 (which produces isolated, elongate crystals) and mnm18 (which produces nearly equidimensional crystals that are usually isolated). Comparison of their FMR spectra indicates that the positive magnetic anisotropy indicated by the spectra of almost all magnetotactic bacteria is a product of chain alignment and particle elongation. We also find correlations between FMR properties and magnetic measurements of coercivity and magnetostatic interactions. FMR thus provides a rapid method for assessing the magnetic properties of assemblages of particles, with applications including screening for samples likely to contain bacterial magnetofossils.

Citation: Kopp, R. E., C. Z. Nash, A. Kobayashi, B. P. Weiss, D. A. Bazylinski, and J. L. Kirschvink (2006), Ferromagnetic resonance spectroscopy for assessment of magnetic anisotropy and magnetostatic interactions: A case study of mutant magnetotactic bacteria, J. Geophys. Res., 111, B12S25, doi:10.1029/2006JB004529.

\section{Introduction}

[2] Ferromagnetic resonance spectroscopy (FMR), a form of electron spin resonance spectroscopy, can serve as a rapid technique for assessing the magnetic anisotropy of and magnetostatic interactions between individual particles in a polycrystalline sample. It is based upon the Zeeman effect, which is the splitting between electron spin energy levels that occurs in the presence of a magnetic field. The Zeeman effect allows a ground state electron to absorb a photon with energy equal to the splitting between the energy states. In a magnetic material, magnetic anisotropy (whether magnetocrystalline, shape, or stress induced) and interparticle interactions contribute to the energy of the particles within a

\footnotetext{
${ }^{1}$ Division of Geological and Planetary Sciences, California Institute of Technology, Pasadena, California, USA.

${ }^{2}$ Photonics Research Institute, National Institute of Advanced Industrial Science and Technology, Osaka, Japan.

${ }^{3}$ Department of Earth, Atmospheric, and Planetary Sciences, Massachusetts Institute of Technology, Cambridge, Massachusetts, USA.

${ }^{4}$ Department of Biochemistry, Biophysics, and Molecular Biology, Iowa State University, Ames, Iowa, USA.
}

Copyright 2006 by the American Geophysical Union. 0148-0227/06/2006JB004529\$09.00 sample and thereby alter the resonance energy. As a result, FMR can be used to probe these parameters [Griscom, 1974, 1981; Kittel, 1948; Kopp et al., 2006; Schlömann, 1958; Weiss et al., 2004].

[3] Techniques for measuring anisotropy and magnetostatic interactions have a number of applications in the field of rock magnetism. The example on which we will focus here is the identification of magnetite produced by magnetotactic bacteria, a topic of great interest for understanding the magnetization of sediments. Fossil magnetotactic bacteria may also serve as paleoenvironmental indicators of both strong magnetic fields and local redox gradients [Chang and Kirschvink, 1989; Kirschvink and Chang, 1984]. These bacteria are a phylogenetically diverse group that biomineralize intracellular crystals of magnetic minerals (magnetite or greigite) which orient the bacteria passively in the geomagnetic field. Natural selection has led these bacteria to optimize the magnetic moment produced for the amount of iron used. Among the traits present in magnetite produced by many magnetotactic bacteria are a narrow distribution of particle sizes within the single-domain stability field, particle elongation, and the arrangement of particles in chains [Thomas-Keprta et al., 2000]. The biophysical problem of keeping strongly magnetic particles aligned in 
a chain may also have driven the evolution of a variety of cytoskeletal supporting mechanisms, including an intracellular "sheath" [Kobayashi et al., 2006], actin-like cytoskeletal filaments [Scheffel et al., 2006] and/or direct attachments to the periplasmic membrane [Komeili et al., 2006].

[4] The adaptive traits possessed by these biogenic magnetic particles at a microscopic level generate distinct magnetic properties that are identifiable with macroscopic techniques. The particles' narrow distribution within the single-domain size range is typically observed in analyses of coercivity spectra, including the measurement of the acquisition of isothermal remanent magnetization and the demagnetization of remanent magnetizations [Chang et al., 1989; Pan et al., 2005]. Egli [2004] used the unmixing of coercivity spectra to determine the biogenic contribution to lacustrine sedimentary magnetization.

[5] Anhysteretic susceptibility, which provides a qualitative measure of inverse interaction strength when comparing single-domain particles of similar volumes [Dunlop et al., 1990; Egli and Lowrie, 2002], has also been used to distinguish bacterial magnetite chains from abiogenic magnetite [Kopp et al., 2006; Moskowitz et al., 1993]. Anhysteretic magnetization is acquired by the application of a small biasing field in the presence of a decaying alternating field. In the absence of thermal effects, noninteracting singledomain particles would have infinite anhysteretic susceptibility; they should become magnetized in the direction of the biasing field as soon as the alternating field decreases below their microcoercivity [Dunlop and Özdemir, 1997; Egli and Lowrie, 2002]. In fact, thermal effects cause more elongate and smaller particles to have lower anhysteretic remanent magnetization (ARM) susceptibility than less elongate or larger single-domain particles [Egli and Lowrie, 2002]. The shielding effects of magnetostatic interactions operating in three dimensions also lower anhysteretic susceptibility.

[6] In many strains of magnetotactic bacteria, however, linear magnetostatic interactions cause an entire chain of particles to behave in a magnetically coherent fashion [Hanzlik et al., 2002; Penninga et al., 1995]. Intact cells of magnetotactic bacteria therefore have low threedimensional magnetostatic interactions and thus relatively high anhysteretic susceptibility, so high anhysteretic remanent magnetization/isothermal remanent magnetization (ARM/IRM) ratios are characteristic of the presence of magnetite chains. Collapsed magnetosome chains, with stronger three-dimensional magnetostatic interactions, have lower ARM/IRM ratios.

[7] Another test that is indicative of the presence of chains is the delta-delta test of Moskowitz et al. [1993], which uses the ratio of magnetization lost upon warming through the $\sim 90-120 \mathrm{~K}$ Verwey transition in saturated samples that have been cooled in a strong field to the magnetization lost after cooling in zero field. While previous data indicate that this test does identify chains of biogenic magnetite [Moskowitz et al., 1993; Weiss et al., 2004], it is susceptible to false negatives and the underlying physical mechanisms are incompletely understood [CarterStiglitz et al., 2004].

[8] Ferromagnetic resonance spectroscopy is capable of rapidly distinguishing biogenic magnetite chains based on three traits: (1) a narrow range of particle size, shape, and arrangement, (2) chain structure, and (3) particle elongation [Kopp et al., 2006; Weiss et al., 2004]. Samples with narrow distributions of size, shape, and arrangement have narrow FMR peaks. Chain structure and particle elongation produce positive uniaxial anisotropy, which can be distinguished from the negative cubic magnetocrystalline anisotropy that dominates isolated, equidimensional magnetite.

[9] Bacterial mutagenesis is a central technique in molecular microbiology. By disabling regions of the genome, it probes the roles of different genes in the production of a phenotype. Our attempts to understand the molecular mechanism of magnetite biosynthesis (which will be described in a follow up paper by C. Z. Nash et al.) led us to create mutant strains of the magnetotactic bacterium Magnetospirillum magneticum strain AMB-1, whose wild-type creates chains of almost equidimensional cubooctahedral crystals. Two of these mutants produce crystals that are usually isolated and are either approximately equidimensional (mutant mnm18) or elongate (mutant mnm13). We used these mutants, along with cells of wildtype AMB-1 and the magnetotactic marine vibrio MV-1, which produces chains of elongate hexaoctahedral crystals, to investigate the contributions of magnetic anisotropy and magnetostatic interactions to ferromagnetic resonance spectra. These different strains allow us for the first time to separate directly the effects of chain structure on FMR and rock magnetic properties from those of single crystal traits.

\section{Methods}

\subsection{Mutagenesis}

[10] To generate the mutants, transposon mutagenesis was performed on AMB-1 following previously described procedures [Komeili et al., 2004]. Mutants were grown up on plates, and single colonies were then picked and grown up in 96-well plates in sealed jars with $2 \%$ oxygen $/ 98 \%$ nitrogen atmospheres. After 3-5 days of growth, weakly magnetic and nonmagnetic mutants were identified by placing the plates on an array of magnets. Mutants that were not drawn toward the side of the well were subcultured for further analysis. For mutant mnm13, sequencing of genomic DNA indicated that an interruption by the introduced transposon occurred in a gene encoding for a hypothetical protein. For mnm18, sequencing indicated that the interruption occurred in a pyruvate/ferredoxin oxidoreductase gene. Time course experiments indicate that mnm18 is a growth defective mutant that takes 1-2 days longer to reach stationary phase than the wild type.

\subsection{Growth Conditions and Lysis}

[11] Cells of strain MV-1 were grown anaerobically with nitrous oxide as the terminal electron acceptor under heterotrophic conditions as previously described [Dean and Bazylinski, 1999]. Cells were harvested at early stationary phase, at a cell density of about $1.5 \times 10^{9}$ cells $/ \mathrm{mL}$, by centrifugation at $5000 \times \mathrm{g}$ at $4^{\circ} \mathrm{C}$ for $10 \mathrm{~min}$ and then resuspended in ice cold artificial seawater containing 20 $\mathrm{mM}$ Tris- $\mathrm{HCl} \mathrm{pH}$ 7.0. Cells were recentrifuged and the resultant pellet of cells was frozen and shipped on dry ice to the California Institute of Technology (Caltech), where it was thawed. A fraction of the cell mass was resuspended in 
Tris buffer, from which point it was subject to the same treatments as AMB-1.

[12] Two liters each of AMB-1 wild-type and mutants mnm13 and mnm18 were grown up to early stationary phase, at a cell density of about $10^{8}$ cells $/ \mathrm{mL}$, using standard culture conditions [Komeili et al., 2004]. The cultures were divided into thirds (A1, A2, and A3 for the wild type; B1, $\mathrm{B} 2$, and $\mathrm{B} 3$ for mnm13; $\mathrm{C} 1, \mathrm{C} 2$, and $\mathrm{C} 3$ for mnm18; V1, $\mathrm{V} 2$, and V3 for MV-1), spun down, and resuspended in $\sim 5 \mathrm{~mL} 100 \mathrm{mM}$ Tris buffer at $\mathrm{pH}$ 7. Five microliters of $\beta$-mercaptoethanol and $\sim 250 \mathrm{mg}$ of sodium dodecyl sulfate (SDS) were added to subsamples A3, B3, C3, and V3. Subsamples A2, A3, B2, B3, C2, C3, V2, and V3 were then subjected to ultrasonication with a Fisher Scientific Sonic Dismembrator 550 for about $6 \mathrm{~min}$, with pulses of $0.5 \mathrm{~s}$ alternating with pauses of equal duration. Ultrasonication should destroy cell membranes while leaving magnetosome membranes intact. SDS treatment should destroy both cell membranes and magnetosome membranes, thereby freeing the magnetite particles from organic structures.

[13] The samples were then spun down, frozen, and freeze-dried. In a set of experiments analogous to the dilution experiments described by Kopp et al. [2006], V2 was diluted at $\sim 1$ part per thousand in sucrose. It was initially measured as sample V2a, then was diluted by mixing with a mortar and pestle for $4 \mathrm{~min}$ to form subsample V2b. Sample V3 was similarly diluted at $\sim 1$ part per thousand as sample V3a, diluted by mixing for 1 min to form sample V3b, and then mixed for four additional minutes to form sample $\mathrm{V} 3 \mathrm{c}$.

\subsection{Electron Microscopy}

[14] Specimens were dispersed on hydrophilic copper transmission electron microscopy (TEM) grids and airdried. The grids were inserted into a beryllium TEM specimen holder for energy dispersive X-ray spectroscopy (EDS) analysis. TEM and high angle annular dark field/ scanning transmission electron microscopy (HAADF/ STEM) images were obtained with a Tecnai G2 F20 Twin (FEI, Holland), operating at $200 \mathrm{kV}$ and equipped with Gatan energy filter GIF2001 and HAADF/STEM detecting unit. The HAADF/STEM/EDS analysis was performed by an EDX detecting unit (EDAX, Inc.). Histograms of particle size/shape distributions were made by measuring the maximum length and widths of magnetite crystals visible in the TEM images in a similar fashion to that of Kirschvink and Lowenstam [1979] and Devouard et al. [1998]. Because of the sharp decay in field strength with distance $\left(1 / \mathrm{r}^{3}\right)$, particles were grouped into chains if they were positioned within less than one grain diameter from an adjacent crystal.

\subsection{Ferromagnetic Resonance Spectra}

[15] Ferromagnetic resonance spectra were acquired using an X-band Bruker ESP 300E EPR Spectrometer housed at Caltech. Except for particularly strong samples (V3a, $\mathrm{V} 3 \mathrm{~b}$, and V3c), microwave power was set at $640 \mu \mathrm{W}$ and spectra were integrated over three sweeps of the applied field from 0 to $600 \mathrm{mT}$. For strong samples, power was set at $64 \mu \mathrm{W}$ and only one spectrum was acquired. To summarize spectral characteristics, we use the empirical parameters developed by Weiss et al. [2004] and Kopp et al. [2006]: $g_{\text {eff, }} A, \Delta B_{\mathrm{FWHM}}$, and $\alpha$. The effective $g$ factor, $g_{\text {eff, }}$ is the $g$ factor associated with maximum absorption which is given by $g_{\text {eff }}=h \nu / \beta B_{\text {eff }}$, where $B_{\text {eff }}$ is the field value of maximum absorption. The asymmetry ratio is defined as $A=\Delta B_{\text {high }} / \Delta B_{\text {low }}$, where $\Delta B_{\text {high }}=B_{\text {high }}-B_{\text {eff, }}$ $\Delta B_{\text {low }}=B_{\text {eff }}-B_{\text {low }}$, and $B_{\text {high }}$ and $B_{\text {low }}$ are the fields of half maximum absorption at low-field and high-field sides of the absorption peak, respectively. The full width at half maximum, $\Delta B_{\mathrm{FWHM}}$, is defined as $\Delta B_{\mathrm{FWHM}}=$

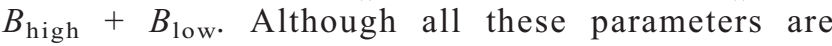
derived from the integrated absorption spectrum, FMR spectra are generally displayed as derivative spectra, which magnify fine detail. The empirical parameter $\alpha$, which serves as a proxy for the line width of symmetric Gaussian broadening caused by factors including heterogeneity of particle size, shape, and arrangement, is defined as $\alpha=$ $0.17 A+9.8 \times 10^{-4} \Delta B_{\mathrm{FWHM}} / \mathrm{mT}$.

[16] The empirical parameters defined above differ from the physical parameters that control the spectral shape ( $g, B_{a n}, K_{2} / K_{1}$, and $\sigma$ ) and which we estimate using the models discussed in section 3. The MATLAB routines used for data analysis and fitting are available in the auxiliary material. $^{1}$

\subsection{Room Temperature Remanent Magnetization Experiments}

[17] Room temperature remanent magnetization experiments were performed using a $2 \mathrm{G}$ Enterprises Superconducting Rock Magnetometer housing in a magnetically shielded room at Caltech and equipped with in-line coils for degaussing, DC pulsing, and applying weak DC biasing fields. Starting with an AF-demagnetized sample, anhysteretic remanent magnetization (ARM) was acquired in a $100 \mathrm{mT}$ alternating field (AF) and a DC biasing field that was raised in $0.05 \mathrm{mT}$ steps to $1 \mathrm{mT}$. The ARM was then removed by stepwise AF demagnetization up to $250 \mathrm{mT}$ in logarithmically spaced steps (where the steps were multiples of $10^{0.1} \mathrm{mT}$ ). The sample was then imparted an isothermal remanent magnetization (IRM) by pulsing with a $100 \mathrm{mT}$ field. This IRM was then removed by stepwise AF demagnetization. Finally, an IRM was imparted stepwise in logarithmically spaced steps up to $980 \mathrm{mT}$ and then removed by AF demagnetization.

[18] To produce coercivity spectra from the stepwise AF and IRM curves, we took the derivative of the curves with respect to the log of the applied field and smoothed the curves with a running average. We report the following parameters: the coercivity of remanence $H_{c r}$, the Cisowski crossover $R$ value, the median acquisition field of IRM $\left(\mathrm{MAF}_{\text {IRM }}\right)$, the median destructive fields of IRM (MDF $\left.{ }_{\text {IRM }}\right)$ and ARM $\left(\mathrm{MDF}_{\mathrm{ARM}}\right)$, and the ARM ratio $k_{\mathrm{ARM}} / \mathrm{IRM}$.

[19] The parameters $H_{c r}$ and $R$ are determined from the IRM stepwise acquisition and demagnetization curves. $H_{c r}$ is the field value at which the two magnetization curves cross, and $R$ is the ratio of magnetization to saturation IRM (SIRM) at that field [Cisowski, 1981]. For noninteracting single-domain particles (or magnetically coherent chains of particles that do not interact with other chains), $R=0.5$, while decreasing values indicate increasing magnetostatic

${ }^{1}$ Auxiliary materials are available in the HTML. doi:10.1029/ 2006JB004529. 
interactions. The median acquisition and destructive fields are defined as the fields required to yield half of the maximum remanent magnetization, where the IRM value is taken from the stepwise IRM curve and the ARM value is taken from the ARM demagnetization curve. We report ARM susceptibility as $k_{\mathrm{ARM}} / \mathrm{IRM}$, the ARM acquired per $\mathrm{A} / \mathrm{m}$ of biasing field (as measured in a biasing field of $0.1 \mathrm{mT}(79.6 \mathrm{~A} / \mathrm{m})$ and an alternating field of $100 \mathrm{mT})$, normalized to the IRM acquired in a field of $100 \mathrm{mT}$.

\subsection{Low-Temperature Rock Magnetic Experiments}

[20] Low-temperature rock magnetic experiments were performed using a Quantum Design Magnetic Properties Measurement System (MPMS) housed in the Molecular Materials Resource Center of the Beckman Institute at Caltech. Following the procedure of Moskowitz et al. [1993], field cooled and zero-field cooled curves were acquired by cooling the sample either in a $3 \mathrm{~T}$ field or in zero field to $5 \mathrm{~K}$, respectively, followed by pulsing with a $3 \mathrm{~T}$ field and then measuring the remanence magnetization during warming to room temperature in zero field. Lowtemperature cycling curves when then acquired by pulsing the sample with a $3 \mathrm{~T}$ field at room temperature and then measuring the remanent magnetization as the sample was cooled to $10 \mathrm{~K}$ and then warmed to room temperature.

[21] The results of the low-temperature experiments are reported as the parameters $\delta_{\mathrm{ZFC}}, \delta_{\mathrm{FC}}$, and $f_{\mathrm{LTC}}$. The parameters $\delta=\left(J_{80 \mathrm{~K}}-J_{150 \mathrm{~K}}\right) / J_{80 \mathrm{~K}}$ were assessed for the zerofield-cooled and field-cooled curves, respectively, where $J_{80 \mathrm{~K}}$ and $J_{150 \mathrm{~K}}$ are the moments measured at $80 \mathrm{~K}$ and $150 \mathrm{~K}$, respectively. A ratio $\delta_{\mathrm{FC}} / \delta_{\mathrm{ZFC}}>2.0$ passes the Moskowitz test and is considered to be an indicator of the presence of magnetosome chains, although partial oxidation and mixing can cause intact chains to fail the test [Moskowitz et al., 1993; Weiss et al., 2004]. Magnetization retained through low-temperature cycling (LTC) is expressed as the memory parameter $f_{\mathrm{LTC}}=J_{\mathrm{LTC}} / J_{0}$, where $J_{0}$ and $J_{\mathrm{LTC}}$ are the room temperature magnetization measured before and after, respectively, cycling the samples to $10 \mathrm{~K}$.

\section{Models}

[22] The models used to fit FMR spectra in this paper are a generalization of prior models [Griscom, 1974, 1981; Kopp et al., 2006]. They derive from the resonance condition [Smit and Beljers, 1955, equation 7]:

$$
\left(\frac{h v}{g \beta}\right)^{2}=\frac{1}{M_{s}^{2} \sin ^{2} \vartheta}\left(\frac{\partial^{2} G}{\partial \vartheta^{2}} \frac{\partial^{2} G}{\partial \varphi^{2}}-\frac{\partial^{2} G}{\partial \vartheta \partial \varphi}\right)
$$

where $h \nu$ is the energy of the microwave photons, $g$ is the spectroscopic $g$ factor of an isolated particle with all anisotropy effects removed, $\beta$ is the Bohr magneton $(9.37 \times$ $\left.10^{-24} \mathrm{~A} \mathrm{~m}^{2}\right), M_{s}$ is the saturation magnetization, $G$ is the free energy of the system, and $\vartheta$ and $\varphi$ are the polar coordinates of the magnetization vector in its minimum energy orientation.

[23] Neglecting thermal energy, which is isotropic and therefore does not appear in equation (1), the free energy $G$ of a system composed of noninteracting, single-domain particles can be written as a sum of the magnetostatic energy $-\mathbf{M} \bullet \mathbf{B}$ and the anisotropy energy. When the

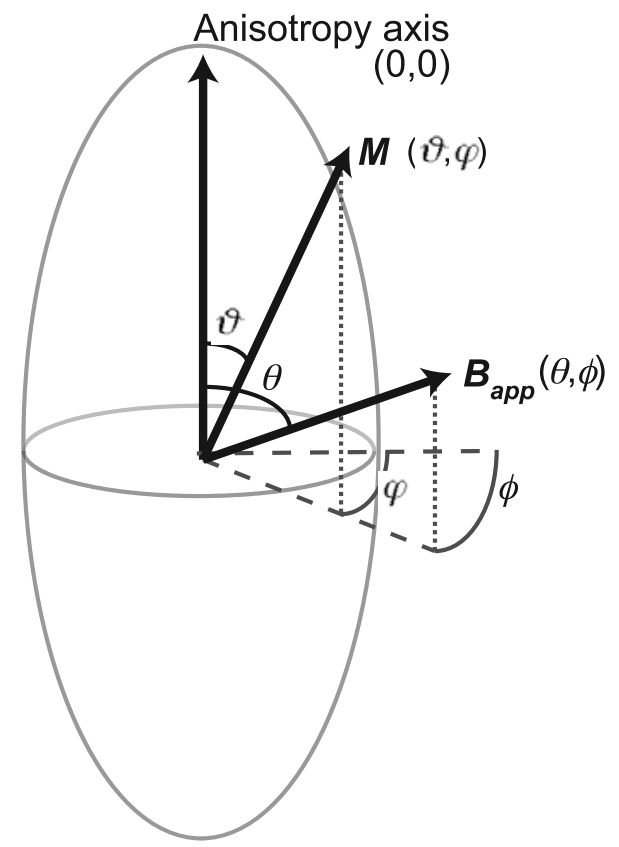

Figure 1. Angles used in the derivation of the resonance conditions. The origin of the reference frame is defined with respect to the anisotropy axis. The applied field $B_{\text {app }}$ is oriented at azimuthal angle $\theta$ and declination $\phi$. The magnetization $M$ is oriented at azimuthal angle $\vartheta$ and declination $\varphi$.

reference frame is defined such that the anisotropy axis is directed along $(\vartheta, \varphi)=(0,0)$ as shown in Figure 1 , the free energy is given by

$$
\begin{aligned}
G= & M_{s}\left\{-B_{a p p}[\sin \vartheta \sin \theta \cos (\phi-\varphi)+\cos \vartheta \cos \theta] .\right. \\
& \left.+\frac{1}{2} B_{a n} F(\vartheta, \varphi)\right\}
\end{aligned}
$$

where $(\theta, \phi)$ are the polar coordinates of the experimentally applied field $B_{\text {app }}$ with respect to the anisotropy axis, $B_{a n}$ is an effective anisotropy field, and $F(\vartheta, \varphi)$ is a geometric factor expressing the variation of the anisotropy energy as a function of the direction of the magnetization vector.

[24] $B_{a n}$ and $F$ vary depending on the source of the anisotropy. For magnetocrystalline anisotropy, $B_{a n}$ is $2 K_{1} / M_{\mathrm{s}}$, where $K_{1}$ is the first-order anisotropy constant (generally written as $K_{1}^{\prime}$ for uniaxial anisotropy). For uniaxial shape anisotropy, $B_{a n}$ is $\mu_{0} M_{s} \Delta N$, where $\mu_{0}$ is the magnetic permeability of free space $\left(4 \pi \times 10^{-7} \mathrm{~N} / \mathrm{A}^{2}\right)$ and $\Delta N$ is the difference between the demagnetization factors $N_{\|}$and $N_{\infty}$ parallel and perpendicular to the elongate axis. For uniaxial anisotropy, regardless of the source,

$$
F(\vartheta)=\sin ^{2} \vartheta+\frac{K_{2}^{\prime}}{K_{1}^{\prime}} \sin ^{4} \vartheta
$$

while for cubic anisotropy,

$$
\begin{aligned}
F(\vartheta, \varphi)= & \sin ^{4} \vartheta \sin ^{2} \varphi \cos ^{2} \varphi+\sin ^{2} \vartheta \cos ^{2} \vartheta \\
& +\frac{K_{2}}{K_{1}} \sin ^{4} \vartheta \cos ^{2} \vartheta \sin ^{2} \varphi \cos ^{2} \varphi
\end{aligned}
$$


where $K_{2}$ is the second-order anisotropy constant (generally written as $K_{2}^{\prime}$ for uniaxial anisotropy) [Dunlop and Özdemir, $1997]$.

[25] By using a first-order approximation to calculate the equilibrium orientation of the magnetization vector and considering only terms that are first order in $B_{a n} / B_{\text {res }}$, we arrive at equation A.3 of Schlömann [1958]:

$$
\left(\frac{h v}{g \beta B_{r e s}}\right)^{2}=1+\frac{B_{a n}}{2 B_{r e s}} a
$$

where $B_{\text {res }}$ is the applied field at which a particle in an arbitrary orientation achieves resonance and

$$
a=\frac{\partial^{2} F}{\partial \vartheta^{2}}+\frac{\partial^{2} F}{\partial \varphi^{2}} \cdot \frac{1}{\sin ^{2} \theta}+\frac{\partial F}{\partial \vartheta} \cot \theta
$$

Solving the quadratic expression in equation (5) yields an expression for $B_{\text {res }}$ as a function of orientation:

$$
B_{r e s}=\sqrt{\left(\frac{h v}{g \beta}\right)^{2}+\left(\frac{a B_{a n}}{4}\right)^{2}}-\frac{a B_{a n}}{4}
$$

For uniaxial anisotropy,

$$
a_{\text {uniaxial }}=6 \cos ^{2} \theta-2+\frac{K_{2}^{\prime}}{K_{1}^{\prime}}\left(16 \cos ^{2} \theta \sin ^{2} \theta-4 \sin ^{4} \theta\right)
$$

while for cubic anisotropy,

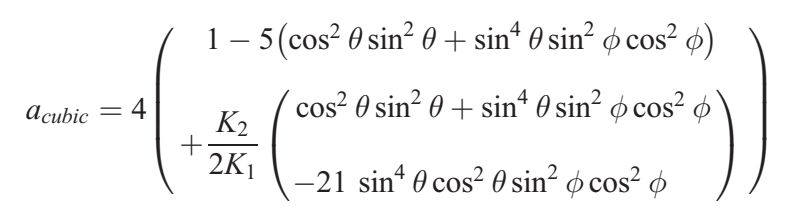

When the second-order anisotropy terms in equation (9) and the second term under the radical in equation (7) are ignored, the resonance conditions thus computed are identical to those of [Griscom, 1974]. When only the second term under the radical is ignored, the cubic anisotropy condition is identical to that of Griscom [1981], except that in equation (9) we drop the third-order anisotropy term in $K_{3}$ introduced by Griscom. The resonance conditions that we have derived are strictly correct to first order in terms of $B_{a n} / B_{\text {res }}$ for dilute powders of single-domain particles.

[26] To compute the powder absorption spectrum at $B_{\text {app }}$, we apply a Gaussian broadening function of line width $\sigma$ and numerically integrate the spectra over all solid angles:

$$
A\left(B_{\text {app }}\right)=\int_{\theta=0}^{\pi / 2} \int_{\phi=0}^{2 \pi} \frac{\exp \left(-\left(B_{\text {app }}-B_{\text {res }}(\theta, \phi)\right)^{2} / 2 \sigma^{2}\right)}{\sqrt{2 \pi} \sigma} d \phi \sin \theta d \theta
$$

The Gaussian broadening incorporates a number of physical effects, including those associated with heterogeneity of size, shape, arrangement, and composition within the sample population. To reflect the physics more accurately, the spectroscopic $g$ factor and the anisotropy parameters ought to have population distributions associated with them individually. However, attempting to fit experimental spectra to a model that employed population distributions for each of these terms would almost always be a problem without a unique solution.

[27] We fit measured spectra to simulated spectra using nonlinear least squares fitting. For each magnetic component included, the models have four parameters that can be adjusted to fit the spectra: $g, B_{a n}, K_{2} / K_{1}$, and $\sigma$. When appropriate, the spectra can be fit to two components, in which case $K_{2} / K_{1}$ is set to zero for both components in order to limit the number of additional degrees of freedom introduced.

[28] For most of the samples, we attempted fits with both cubic and uniaxial models, as well as models combining two uniaxial components, two cubic components, or a uniaxial component and a cubic component. Except when $\left|B_{a n}\right| \gg \sigma$, substituting a cubic component for a uniaxial component did not significantly improve or degrade the goodness of the fit. We suspect this is because the Gaussian broadening conceals the underlying physics in a fashion that makes it difficult to discriminate between samples best fit with a cubic component and those best fit with a uniaxial component.

[29] The substitution often had only slight effects on the fitted parameters as well, but sometimes did vary the parameters outside the confidence intervals on the uniaxial fits. Because the anisotropy field expected from the cubic magnetocrystalline anisotropy of stoichiometric magnetite $\left(K_{1}=-1.35 \times 10^{4} \mathrm{~J} / \mathrm{m}^{3}, M_{s}=480 \mathrm{kA} / \mathrm{m}\right)$ is about $-56 \mathrm{mT}$ [Dunlop and Özdemir, 1997], we report the fitted parameters using cubic anisotropy for components with $B_{a n}$ between approximately -56 and $0 \mathrm{mT}$. (The dominant components of samples $\mathrm{C} 1$ and $\mathrm{C} 2$ are the only components that fit this criterion.) The underlying physics in fact reflects neither purely uniaxial nor purely cubic anisotropy but a more complicated combination of approximately uniaxial shape anisotropy and cubic magnetocrystalline anisotropy, which would be reflected in a more complete form of equation (2). Some of the Gaussian broadening likely results from our simplified treatment of the anisotropy.

[30] The decision as to whether to represent a measured spectrum with a one-component spectrum or the sum of two model spectra was made heuristically, based upon the level of the improvement of fit when a second spectrum was added, how physically realistic the two spectra identified by the fitting routine are, and the size of the confidence intervals around fit parameters. In interpreting the models, it is important to remember that, if properly modeled, multiple components in a fit reflect multiple end-members mixed together (e.g., isolated particles and particles in chains, or particles in chains and particles in clumps); they do not reflect multiple aspects of the anisotropy of a single end-member.

\section{Results}

\subsection{Electron Microscopy}

[31] Consistent with the work of Devouard et al. [1998], our TEM images indicate that MV-1 produces chains of 

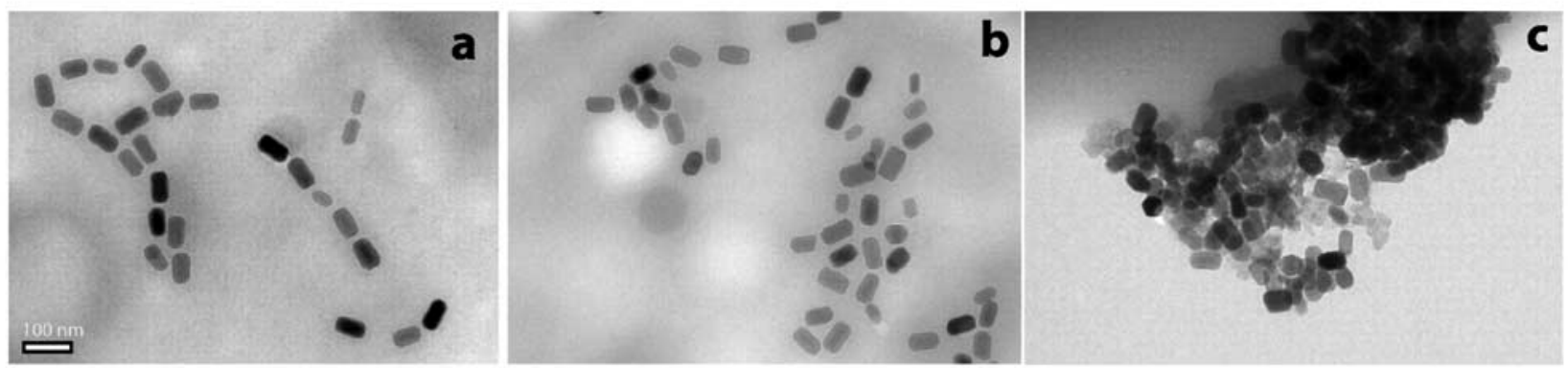

Figure 2. Transmission electron micrographs of MV-1. (a) Sample V1, untreated, (b) sample V2a, ultrasonicated, and (c) sample V3a, lysed with SDS. Scale bar is $100 \mathrm{~nm}$.

magnetite crystals with a mean single-crystal length of $\sim 75 \mathrm{~nm}$ and a mean length-to-width ratio of $\sim 1.8$ (Figure 2). The untreated cells of MV-1 that we measured experienced some chain collapse, perhaps due to the freezing of the sample. As can be seen in Figure 2a, some chains collapsed into zero stray field loop configurations, while in other chains some of the particles have fallen into sideby-side arrangements. About $10 \%$ of the crystals appear sufficiently separated from other crystals to be magnetically isolated. Collapse features are greatly enhanced by ultrasonication (Figure 2b). Few of the chains in the ultrasonicated sample V2 are unaffected; most are bent or interwoven with other chains. Only a small number of crystals are magnetically isolated. Treatment with SDS (sample V3) led to near complete collapse of chains into clumps (Figure 2c).

[32] There appears to be a greater tendency for chain collapse to occur in strain MV-1 than in M. magnetotacticum strain MS-1, a strain related to AMB-1 that produces more equidimensional particles than MV-1 [Kobayashi et al., 2006]. In the case of MS-1, ultrasonication does not produce many side-by-side crystal pairs. Instead, ultrasonication of MS-1 tends to cause chains to string together in a head-to-tail fashion. The difference between the collapse styles of MV-1 and MS-1 is likely attributable to the energetic differences between elongate and equidimensional particles.

[33] Cells of wild-type AMB-1 produce magnetite particles with a mean particle length of $\sim 35 \mathrm{~nm}$ and lengthto-width ratio $\sim 1.2$ (Figures $3 \mathrm{a}, 3 \mathrm{~d}$, and $3 \mathrm{f}$ ). In powder A1, derived from freeze-dried wild-type AMB-1, $\sim 65 \%$ by volume of the crystals we measured were in chains of at least 2 particles and $\sim 35 \%$ were isolated. Previous observations of whole cells of wild-type AMB-1 indicate that single cells often produce chains with segments of anywhere between 1 and 21 crystals separated by gaps. The presence of isolated crystals in the freeze-dried powder is likely due to a combination of gaps in chains produced by single cells and disaggregation during the freeze-drying process.

[34] Cells of the AMB-1 mutant mnm13 produce elongated crystals, with a mean length of $\sim 25 \mathrm{~nm}$ and a mean length-to-width ratio of $\sim 1.5$ (Figures $3 \mathrm{~b}, 3 \mathrm{e}$, and $3 \mathrm{~h}$ ). About $90 \%$ by volume of the crystals produced by mnm13 are isolated and $\sim 10 \%$ are in chains of 2 or more particles. Among particles with a length $>25 \mathrm{~nm}$, which dominate by volume and control the magnetic properties, the mean length-to-width ratio is $\sim 1.75$. Some of the bias toward greater elongation in larger crystals is likely observational; an elongate particle, viewed down the axis of elongation, appears to have a width/length ratio of 1 and a shorter length than its true length.

[35] Cells of the mutant mnm18 produce more equidimensional crystals, similar to those produced by the wild type, with a mean length of $\sim 40 \mathrm{~nm}$ and a mean lengthto-width ratio of $\sim 1.2$ (Figures $3 \mathrm{c}, 3 \mathrm{f}$, and $3 \mathrm{i}$ ). By volume, $\sim 65 \%$ of the particles are isolated and $\sim 35 \%$ are in chains. Though most of the chains consist of only two particles, they can grow significantly longer. The longest mnm18 chain we observed consisted of seven particles, which suggests that a small fraction of mnm18 cells exhibit the wild-type phenotype.

\subsection{Ferromagnetic Resonance Spectroscopy}

[36] Our measurements of the FMR spectra of intact MV-1 and wild-type AMB-1 agree with those of Weiss et al. [2004], exhibiting distinctive asymmetric spectra that are extended in the low-field direction. MV-1 has a broader spectrum than AMB-1, which reflects the greater anisotropy of its magnetite chains, generated by particle elongation as well as chain alignment (Table 1 and Figures 4 and 5). MV-1 also has three characteristic maxima in the derivative spectrum, seen in samples V1, V2a, and V2b at $\sim 180$, $\sim 300$, and $\sim 350 \mathrm{mT}$. Our one-component model spectra are unable to reproduce this trait. Our attempts to fit these three spectra with two-component models, however, yielded disparate secondary fit components with no clear physical interpretation. This disparity suggests the values thus determined were artifacts, and we therefore report the singlecomponent fits in Table 2. A more complete physical model capable of including multiple sources of anisotropy might explain the distinctive triple maxima of MV-1 spectra.

[37] On the basis of the demagnetization factors derived by Osborn [1945] and assuming that the anisotropy is dominated by uniaxial shape anisotropy, the $B_{a n}$ value of $171 \mathrm{mT}$ fitted to the spectrum of intact MV-1 (sample V1) is that expected from prolate spheroids of stoichiometric magnetite $\left(M_{s}=480 \mathrm{kA} / \mathrm{m}\right)$ with length-to-width ratios of $\sim 2.35$. The calculated ratio is significantly larger than that observed for individual particles under TEM and therefore 

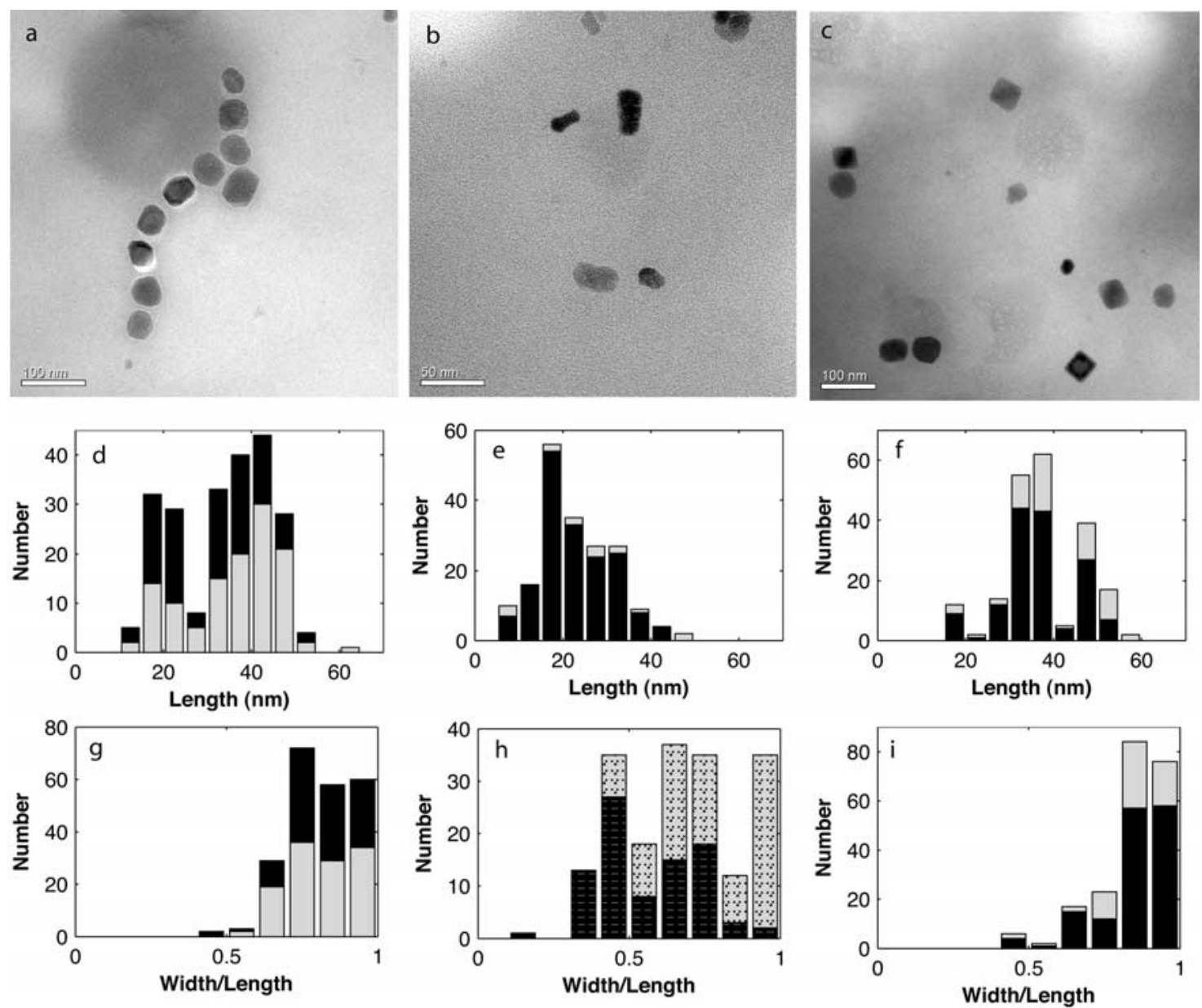

Figure 3. Transmission electron micrographs of and summary statistics for AMB-1 strains. (a-c) TEM images of freeze-dried powders of wild type (Figure 3a), mnm13 (Figure 3b), and mnm18 (Figure 3c). In Figures 3a and 3c, scale bar is $100 \mathrm{~nm}$; in Figure 3b, scale bar is $50 \mathrm{~nm}$. (d-f) Histograms of particle length for magnetite produced by wild type (Figure 3d), mnm13 (Figure 3e), and mnm18 (Figure 3f). ( $g-\mathrm{i})$ Histograms of particle width/length ratios for magnetite produced by wild type (Figure $3 \mathrm{~g}$ ), mnm13 (Figure 3h), and mnm18 (Figure 3i). In Figures 3d-3g and 3i, dark bars represent particles in chains and light bars represent isolated particles. In Figure $3 \mathrm{~h}$, dark bars represent particles with length $\geq 25 \mathrm{~nm}$ and light bars represent particles with length $<25 \mathrm{~nm}$.

likely reflects the joint contribution of particle elongation and chain structure. The large, positive $B_{a n}$ value indicates that the negative contribution of magnetocrystalline anisotropy is overwhelmed by the positive contributions of shape anisotropy and chain structure.

[38] Ultrasonication of MV-1 broadens the FMR spectrum, with $\Delta B_{\mathrm{FWHM}}$ increasing from $127 \mathrm{mT}$ in sample V1 to 219 in sample V2a. This broadening, which is reflected in the spectral fits by an increase of $\sigma$ from $17 \mathrm{mT}$ to $27 \mathrm{mT}$ despite a slight decline in $B_{a n}$, suggests an increase in the heterogeneity of particle arrangement without the formation of strongly interacting clumps. Although dilution (sample V2b) produces a significant increase in anhysteretic susceptibility (see discussion below), it results in little change in the FMR spectrum.

[39] In contrast, lysis of MV-1 cells with SDS produces a drastic change in the FMR spectrum, as it causes the particles to collapse into clumps. The FMR spectra of these clumps, like the FMR spectra of similarly treated AMB-1 observed by Weiss et al. [2004] and Kopp et al. [2006] and in the present work, are broad and exhibit high-field extended asymmetry reflective of a negative effective anisotropy field. The negative anisotropy may reflect the anisotropy of the surface of particle clumps or the oblateness of the clumps. Although modeling clumps with expressions derived for isolated particles is far from ideal, the fitted $B_{a n}$ value of $-120 \mathrm{mT}$ corresponds to that predicted for oblate spheroids with a length-to-width ratio of $\sim 0.62$. [Griscom et al., 1988] observed similar traits in the spectra of powders of magnetite nanoparticles exhibiting planar interactions.

[40] Subsequent dilution causes the gradual reappearance of positive anisotropy, again as in the case of AMB-1 [Kopp et al., 2006]. After $1 \mathrm{~min}$ of dilution, the spectrum is best fit by a two-component model, with $84 \%$ of the absorption caused by a component with $B_{a n}$ of $-130 \mathrm{mT}$ and $16 \%$ caused by a component with $B_{a n}$ of $157 \mathrm{mT}$. The former component likely corresponds to particles in clumps, while 
Table 1. Measured Ferromagnetic Resonance Parameters

\begin{tabular}{|c|c|c|c|c|c|c|}
\hline Sample & Strain & Treatment & $g_{\text {eff }}$ & $A$ & $\Delta B_{\mathrm{FWHM}}, \mathrm{mT}$ & $A$ \\
\hline A1 & AMB-1 wild type & untreated & 2.01 & 0.76 & 87 & 0.21 \\
\hline A2 & AMB-1 wild type & sonicated & 2.02 & 0.79 & 84 & 0.22 \\
\hline A3 & AMB-1 wild type & SDS & 2.31 & 1.17 & 206 & 0.40 \\
\hline B1 & AMB-1 mnm13 & untreated & 2.02 & 0.88 & 91 & 0.24 \\
\hline B2 & AMB-1 mnm13 & sonicated & 2.01 & 0.86 & 95 & 0.24 \\
\hline B3 & AMB-1 mnm13 & SDS & 2.02 & 0.83 & 107 & 0.25 \\
\hline $\mathrm{C} 1$ & AMB-1 mnm18 & untreated & 2.07 & 1.13 & 80 & 0.27 \\
\hline $\mathrm{C} 2$ & AMB-1 mnm18 & sonicated & 2.07 & 1.16 & 79 & 0.27 \\
\hline $\mathrm{C} 3$ & AMB-1 mnm18 & SDS & 2.07 & 0.78 & 151 & 0.28 \\
\hline V1 & MV-1 & untreated & 1.78 & 0.35 & 127 & 0.18 \\
\hline V2a & MV-1 & sonicated & 1.84 & 0.29 & 219 & 0.26 \\
\hline $\mathrm{V} 2 \mathrm{~b}$ & MV-1 & sonicated, $4 \mathrm{~m}$ dilution & 1.85 & 0.30 & 206 & 0.25 \\
\hline V3a & MV-1 & SDS & 2.58 & 1.77 & 244 & 0.54 \\
\hline V3b & MV-1 & SDS, $1 \mathrm{~m}$ dilution & 2.54 & 1.62 & 218 & 0.49 \\
\hline $\mathrm{V} 3 \mathrm{c}$ & MV-1 & SDS, $5 \mathrm{~m}$ dilution & 1.86 & 0.25 & 208 & 0.25 \\
\hline
\end{tabular}

the latter component likely corresponds to particles that are either isolated or in strings. After $5 \mathrm{~min}$ of dilution, the component with positive anisotropy dominates the spectrum (Figure 5).

[41] The spectrum of untreated cells of mnm13 is not markedly different from that of wild-type AMB-1 (Figure 5). Although the empirical asymmetry parameter $A$ for mnm13 reflects a lesser degree of asymmetry than the wild type, this represents a failure of the empirical parameter; the fitted spectra reveal that mnm13 in fact has a somewhat stronger anisotropy field than the wild type, which reflects the particle elongation. The wild type has a fitted $B_{a n}$ of $69 \mathrm{mT}$, corresponding to a length-to-width ratio of $\sim 1.35$, while mnm13 has a fitted $B_{a n}$ of $91 \mathrm{mT}$, corresponding to a lengthto-width ratio of $\sim 1.50$. As with $\mathrm{MV}-1$, the ratio calculated for the wild type exceeds the value observed under TEM for individual particles, likely due to the effect of the chain

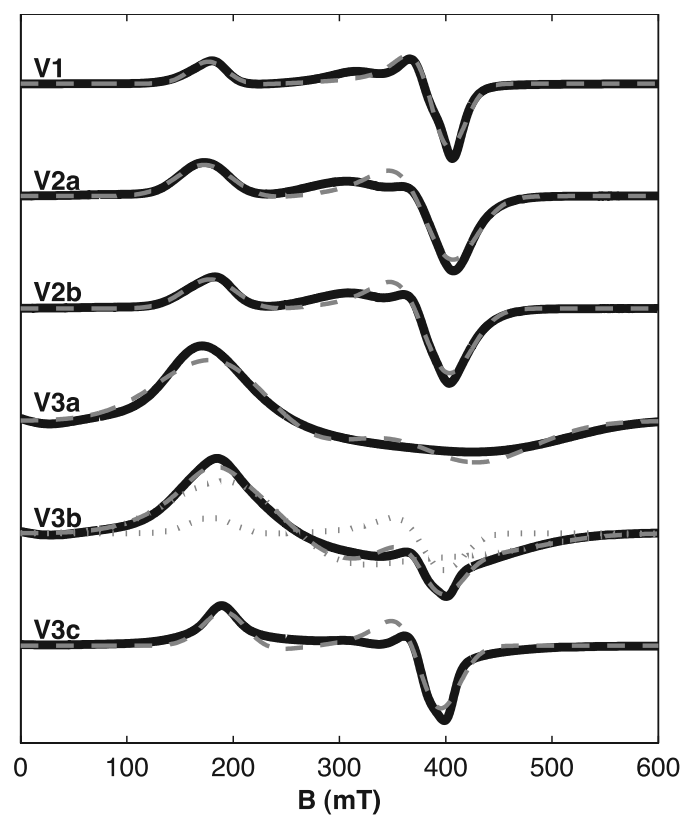

Figure 4. FMR spectra of MV-1. Solid lines show measured spectra, dashed lines show fitted spectra, and dotted lines show the spectra of the individual fit components for two-component fits. structure in increasing $B_{a n}$. In contrast, the ratio calculated for mnm13 corresponds to that observed under TEM.

[42] Both sonication and lysis with SDS cause a slight increase in the fitted anisotropy field of mnm13, which may reflect the formation of short strings of particles. In contrast,

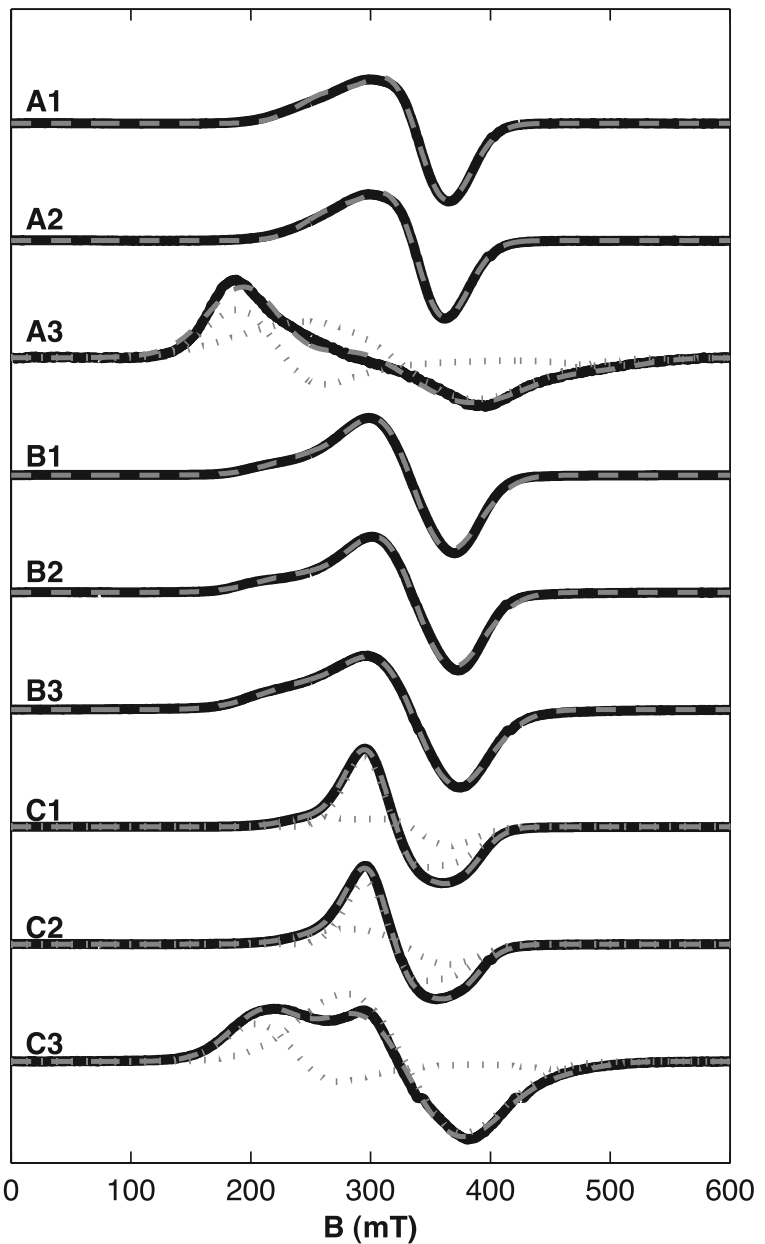

Figure 5. FMR spectra of AMB-1 wild type (A1-A3), mnm13 (B1-B3), and mnm18 (C1-C3). Solid lines show measured spectra, dashed lines show fitted spectra, and dotted lines show the spectra of the individual fit components for two-component fits. 
Table 2. Ferromagnetic Resonance Spectral Fits ${ }^{\mathrm{a}}$

\begin{tabular}{|c|c|c|c|c|c|}
\hline Sample & Component Weight, \% & $g$ & $B_{a n}, \mathrm{mT}$ & $K_{2} / K_{1}$ & $\sigma, \mathrm{mT}$ \\
\hline A1 & 100 & $2.07 \pm 0.00$ & $69.1 \pm 0.8$ & $-0.12 \pm 0.01$ & $24.2 \pm 0.2$ \\
\hline A2 & 100 & $2.07 \pm 0.00$ & $63.6 \pm 0.7$ & $-0.13 \pm 0.01$ & $23.5 \pm 0.2$ \\
\hline \multirow[t]{2}{*}{ A3 } & 61 & $2.15 \pm 0.03$ & $87.4 \pm 10.1$ & & $55.5 \pm 2.4$ \\
\hline & 39 & $2.38 \pm 0.01$ & $-171.2 \pm 4.3$ & & $31.7 \pm 1.5$ \\
\hline B1 & 100 & $2.08 \pm 0.00$ & $90.9 \pm 2.5$ & $-0.32 \pm 0.02$ & $31.1 \pm 0.3$ \\
\hline $\mathrm{B} 2$ & 100 & $2.09 \pm 0.00$ & $104.3 \pm 2.5$ & $-0.31 \pm 0.01$ & $31.8 \pm 0.2$ \\
\hline B3 & 100 & $2.10 \pm 0.00$ & $99.7 \pm 1.5$ & $-0.23 \pm 0.01$ & $34.2 \pm 0.2$ \\
\hline \multirow[t]{2}{*}{$\mathrm{C} 1$} & 70 & $2.05 \pm 0.00$ & $-47.3 \pm 0.8$ & & $19.2 \pm 0.1$ \\
\hline & 30 & $2.12 \pm 0.01$ & $76.1 \pm 1.1$ & & $22.1 \pm 0.8$ \\
\hline \multirow[t]{2}{*}{$\mathrm{C} 2$} & 57 & $2.06 \pm 0.00$ & $-43.0 \pm 0.6$ & & $18.4 \pm 0.2$ \\
\hline & 43 & $2.09 \pm 0.01$ & $64.1 \pm 3.1$ & & $28.2 \pm 0.6$ \\
\hline \multirow[t]{2}{*}{$\mathrm{C} 3$} & 68 & $2.05 \pm 0.01$ & $50.2 \pm 5.4$ & & $43.1 \pm 0.8$ \\
\hline & 32 & $2.34 \pm 0.01$ & $-142.3 \pm 3.3$ & & $30.4 \pm 0.9$ \\
\hline V1 & 100 & $2.21 \pm 0.01$ & $170.9 \pm 2.6$ & $-0.03 \pm 0.01$ & $17.3 \pm 0.3$ \\
\hline V2a & 100 & $2.26 \pm 0.01$ & $164.0 \pm 3.2$ & $0.01 \pm 0.01$ & $26.7 \pm 0.6$ \\
\hline $\mathrm{V} 2 \mathrm{~b}$ & 100 & $2.24 \pm 0.01$ & $160.4 \pm 2.7$ & $0.00 \pm 0.01$ & $24.9 \pm 0.4$ \\
\hline V3a & 100 & $2.35 \pm 0.01$ & $-120.0 \pm 2.3$ & $0.23 \pm 0.02$ & $56.9 \pm 0.8$ \\
\hline \multirow[t]{2}{*}{ V3b } & 84 & $2.37 \pm 0.01$ & $-129.9 \pm 2.6$ & & $58.3 \pm 0.9$ \\
\hline & 16 & $2.24 \pm 0.01$ & $157.4 \pm 2.5$ & & $21.2 \pm 0.7$ \\
\hline $\mathrm{V} 3 \mathrm{c}$ & 100 & $2.24 \pm 0.01$ & $132.6 \pm 3.4$ & $0.08 \pm 0.02$ & $20.6 \pm 0.6$ \\
\hline
\end{tabular}

${ }^{\mathrm{a}}$ The dominant components of $\mathrm{C} 1$ and $\mathrm{C} 2$ are modeled using cubic anisotropy. All other components are modeled using uniaxial anisotropy.

while sonication has only slight effect on the wild type, treatment of the wild type with SDS leads to a broader spectrum that is best fit by a two-component model in which $61 \%$ of particles have positive anisotropy $\left(B_{a n}=87 \mathrm{mT}\right)$ and $39 \%$ have negative anisotropy $\left(B_{a n}=-171 \mathrm{mT}\right)$. The latter component may reflect clumping. The absence of clumps in SDS-treated mnm13 suggests that the greater diluteness of the particles prevents them from clumping.

[43] The mutant mnm18 has an extremely distinctive spectrum (Figure 5). It is the only untreated magnetotactic bacterium measured so far that has $A>1$, which reflects the negative magnetocrystalline anisotropy of isolated particles of equidimensional magnetite. It provides the best example of a spectrum that can be fitted as a mixture, as it is the mixture of two components with clear physical interpretations corresponding to TEM observations. The intact mnm18 is a mixture composed $70 \%$ of a component with negative anisotropy $\left(B_{a n}=-47 \mathrm{mT}\right)$ and $30 \%$ of a positive anisotropy component with parameters closely resembling those of the wild type $\left(B_{a n}=76 \mathrm{mT}\right)$. From the FMR data, we can predict that, by volume, the sample consists $70 \%$ of isolated crystals and $30 \%$ of chains of at least 2 crystals in length. These proportions are in close agreement with the values $(65 \%$ and $35 \%)$ estimated from the TEM images, which confirms the proposal by Weiss et al. [2004] that the uniquely asymmetric FMR spectrum of magnetotactic bacteria results primarily from the alignment of crystals in chains. We can also use this composition to unmix the isolated crystals from the other rock magnetic parameters, taking the properties of the wild type to represent those of the fraction in chains.

[44] The isolated component has a narrower Gaussian line width $(\sigma=20 \mathrm{mT})$ than is typical of most magnetotactic bacteria, which may reflect that lesser degree of heterogeneity possible with isolated crystals than with arrangements of crystals. The anisotropy measured for isolated crystals of mnm18 is slightly less than that expected for isolated crystals of stoichiometric magnetite dominated by cubic anisotropy, which would have $B_{a n}$ of about $-56 \mathrm{mT}$. The reduced anisotropy constant $\left(K_{1} \approx 1.1 \times 10^{4} \mathrm{~J} / \mathrm{m}^{3}\right)$ may result from minor nonstochiometry $(\sim 0.4 \%$ cation depletion) [Ka kol and Honig, 1989], which is consistent with the reduced Verwey transition temperature of $\sim 100 \mathrm{~K}$ observed in AMB-1 magnetite (Figure 6e) [Muxworthy and McClellan, 2000].

[45] Sonication of mnm18 cells leads to a slight increase in the proportion in chains, while treatment with SDS drastically alters the spectrum. SDS-treated cells of mnm18 come to resemble those of the wild-type more closely, because the sample becomes dominated by short linear strings of particles, the anisotropy of which is controlled primarily by particle arrangement. The fitted spectrum consists $68 \%$ of a component with positive anisotropy $\left(B_{a n}=50 \mathrm{mT}\right)$ and $32 \%$ of a component with strong negative anisotropy $\left(B_{a n}=-142 \mathrm{mT}\right)$ comparable to those of clumps formed in SDS treatment of wild-type AMB-1 and MV-1. Thus the comparison of the unmixed components of intact and SDS-treated mnm18 provides powerful insight into the role of chain formation in controlling the magnetic properties of magnetotactic bacteria.

\subsection{Isothermal Remanent Magnetization}

[46] The room temperature IRM acquisition coercivity spectra for cells of wild-type AMB- 1 and MV-1, regardless of treatment, agree in general shape, though not in precise parameterization, with the biogenic soft and biogenic hard components recognized by Egli [2004] (Figure 6a and Table 3). MV-1 has a narrow peak centered at a median field of $55 \mathrm{mT}$, while AMB-1 has a broader peak centered at $27 \mathrm{mT}$. The mutant mnm13 is slightly softer than the wild type (median field of $23 \mathrm{mT}$ ), which may be due to the smaller volume of mnm13 particles. The mutant mnm18 is both softer and has a broader spectrum than the other strains (median field of $16 \mathrm{mT}$ ). When FMR analyses and TEM observations are used to guide the unmixing of the chains and solitary particles in mnm18, the solitary particles are revealed to have a spectrum with a median coercive field of $11 \mathrm{mT}$. The drastic difference between the isolated, equidimensional 

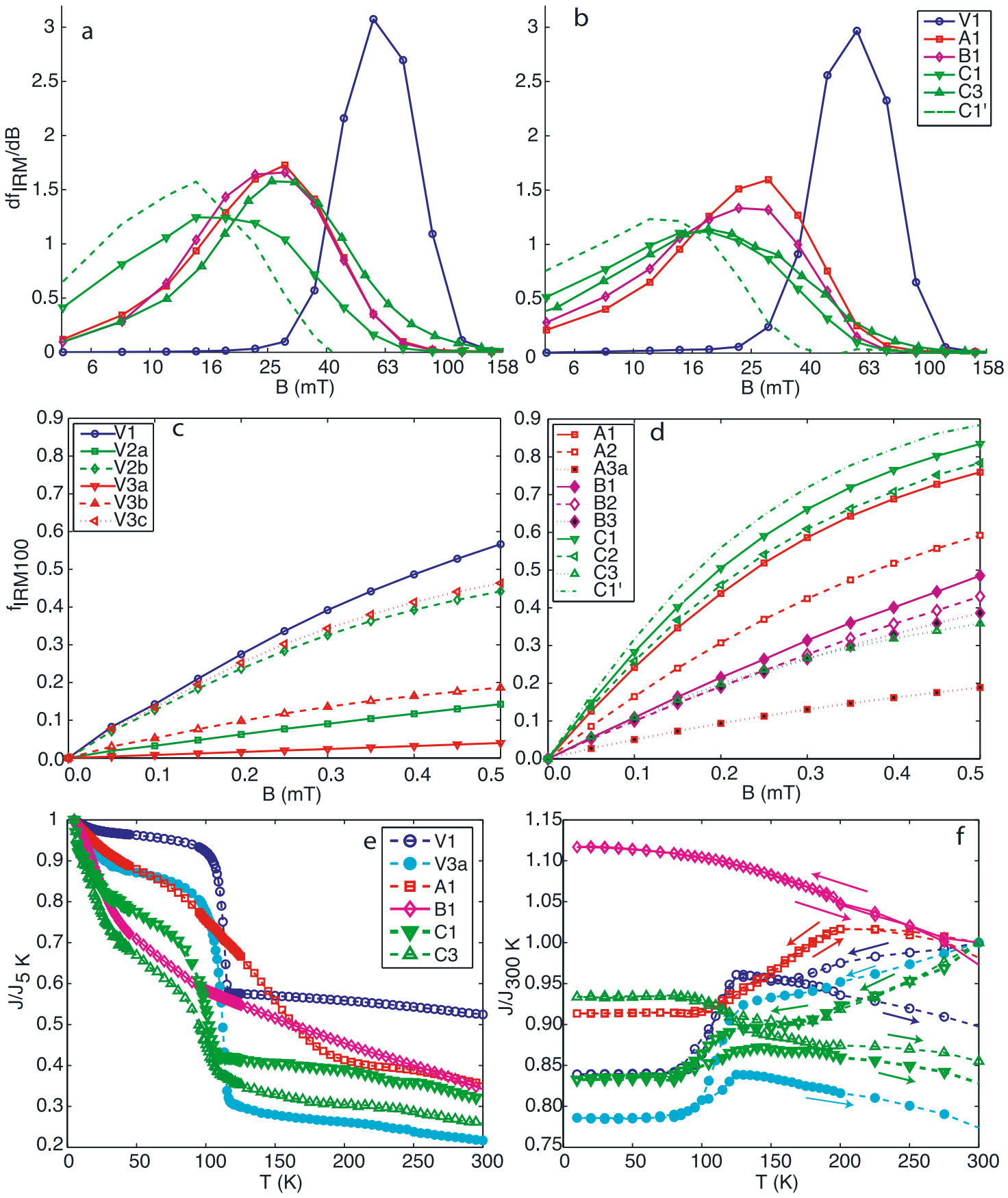

Figure 6. Rock magnetic measures of selected samples. (a-b) Coercivity spectra determined from stepwise IRM acquisition (Figure 6a) and stepwise AF demagnetization of IRM (Figure 6b). The dashed line $\mathrm{C}^{\prime}$ ' in Figure $6 \mathrm{a}, 6 \mathrm{~b}$, and $6 \mathrm{~d}$ indicates the unmixed isolated particle component of $\mathrm{C} 1$, produced using the proportions of isolated and chain components determined from the FMR spectra to remove the chain component. (c-d) ARM acquisition curves of MV-1 (Figure 6c) and AMB-1 wild type and mutants (Figure 6d). (e-f) Low-temperature demagnetization curves. Figure 6e shows the demagnetization upon warming of a magnetization acquired by saturation at $5 \mathrm{~K}$ of samples cooled in a $3 \mathrm{~T}$ field. Magnetization values are shown normalized to the magnetization at $5 \mathrm{~K}$. Figure $6 \mathrm{f}$ shows the demagnetization upon cooling and subsequent warming of a magnetization acquired by saturation at $300 \mathrm{~K}$. Magnetization values are shown normalized to the initial room temperature magnetization.

particles produced by mnm18 and the elongate particles of mnm13, as well as the chains of equidimensional particles in the wild-type cells, highlights the role of these traits in stabilizing the magnetic moments of magnetotactic bacteria.
[47] For all samples of unlysed cells of AMB-1, both wildtype and mutant, acquisition and demagnetization curves align fairly closely (Figure 6b); Cisowski $R$ values are all $\geq 0.42$, and the median destructive field falls within $5 \mathrm{mT}$ of 
Table 3. Room Temperature Rock Magnetic Parameters ${ }^{\mathrm{a}}$

\begin{tabular}{|c|c|c|c|c|c|c|c|}
\hline Sample & $H_{c r}, \mathrm{mT}$ & $R$ & MAF of IRM, mT & MDF of IRM, mT & MDF of ARM, mT & $k_{\mathrm{ARM}} / \mathrm{IRM}, \mathrm{mm} / \mathrm{A}$ & Predicted Switching Field, $\mathrm{mT}$ \\
\hline A1 & 24.0 & 0.44 & 26.5 & 21.6 & 22.2 & 2.93 & 30.3 \\
\hline A2 & 22.0 & 0.43 & 24.0 & 19.8 & 21.1 & 2.07 & 27.8 \\
\hline A3 & 16.6 & 0.29 & 24.1 & 10.2 & 17.4 & 0.64 & 59.8 \\
\hline B1 & 21.3 & 0.42 & 23.4 & 19.3 & 19.3 & 1.37 & 31.0 \\
\hline B2 & 23.9 & 0.42 & 26.6 & 22.2 & 22.3 & 1.26 & 35.7 \\
\hline B3 & 26.0 & 0.39 & 30.7 & 21.8 & 24.2 & 1.29 & 38.2 \\
\hline $\mathrm{C} 1$ & 14.5 & 0.47 & 15.7 & 13.8 & 13.7 & 3.55 & 26.7 \\
\hline $\mathrm{C} 1$, & 10.7 & 0.44 & 10.6 & 9.9 & 10.5 & 3.99 & 22.3 \\
\hline $\mathrm{C} 2$ & 14.7 & 0.44 & 16.6 & 13.8 & 13.8 & 3.25 & 24.4 \\
\hline $\mathrm{C} 3$ & 21.7 & 0.35 & 26.7 & 16.6 & 21.9 & 1.38 & 39.8 \\
\hline V1 & 57.8 & 0.42 & 55.3 & 61.2 & 65.8 & 1.79 & 82.9 \\
\hline V2a & 55.1 & 0.27 & 63.3 & 45.9 & 58.1 & 0.41 & 82.8 \\
\hline $\mathrm{V} 2 \mathrm{~b}$ & 48.4 & 0.31 & 55.0 & 41.7 & 49.8 & 1.59 & 80.2 \\
\hline V3a & 28.4 & 0.14 & 43.2 & 17.0 & 24.7 & 0.10 & 73.8 \\
\hline V3b & n.d. & n.d. & n.d. & n.d. & 52.7 & 0.66 & 67.2 \\
\hline V3c & 52.3 & 0.34 & 52.5 & 46.5 & 56.3 & 1.68 & 71.6 \\
\hline
\end{tabular}

${ }^{\mathrm{a}} \mathrm{Cl}^{\prime}$ is the unmixed end-member of $\mathrm{C} 1$ composed of isolated particles. Stepwise IRM curves were not measured for V3b. Predicted switching field is calculated from the FMR fit parameters as described in the text; n.d., not determined.

the median acquisition field. This is not the case for the SDStreated mutants and both the SDS-treated and the ultrasonicated wild types, which reflects greater interparticle magnetostatic interactions in the wild types than in the mutants. Notably, the IRM acquisition curve of SDS-treated mnm18 closely resembles that of the wild type (median field of $27 \mathrm{mT}$ ), while the demagnetization curve remains closer to that of the untreated mnm18. As FMR data indicate the formation of linear strings of particle in the SDS-treated mnm18, the observation may suggest that IRM acquisition coercivity is more strongly affected by chain structures than is demagnetization coercivity.

\subsection{Anhysteretic Remanent Magnetization}

[48] The ARM acquisition curves for wild-type AMB-1 and $\mathrm{MV}-1$ are consistent with previous measurements [Moskowitz et al., 1993, 1988] (Figures 6c and 6d and Table 3). MV-1 has markedly lower anhysteretic susceptibility than AMB-1. Two factors likely contribute to this difference. First, as seen in the TEM images, untreated MV-1 has undergone a greater degree of chain collapse than untreated AMB-1, due to the intrinsic instability of chains of elongate particles. The increased three-dimensional magnetostatic interactions in collapsed chains serve to lower ARM susceptibility. Second, elongate particles have a higher switching field and thus lower intrinsic ARM susceptibility than more equidimensional particles of the same volume [see Egli, 2003, Figure 11].

[49] The pattern of variation of ARM susceptibility of lysed MV-1 shows some notable differences from parallel experiments previously reported for AMB-1 [Kopp et al., 2006]. For both strains, ultrasonicated bacteria exhibit a lower susceptibility than untreated bacteria and a higher susceptibility than SDS-treated bacteria. However, whereas dilution of ultrasonicated AMB-1 produced little change in ARM susceptibility, dilution of ultrasonicated MV-1 produces significant change. Undiluted ultrasonicated MV-1 exhibits a similar susceptibility to SDS-treated MV-1 diluted for $1 \mathrm{~min}$, and ultrasonicated MV-1 diluted for 4 min exhibits a similar susceptibility to SDS-treated MV-1 diluted for $5 \mathrm{~min}$. The difference between the strains again likely reflects differences in collapse style between equidimensional particles and elongate particles; the strings produced by ultrasonication of AMB-1 are less likely to be reconfigured during dilution than the meshes produced by ultrasonication of MV-1.

[50] The crystals produced by mnm13 have even lower anhysteretic susceptibility than MV-1, a reflection of the combined influence of their elongation and their smaller size. In fact, their ARM susceptibility lies significantly above what would be predicted based on TEM measurements. Egli and Lowrie [2002] calculate that a particle with a length-to-width ratio of 1.9 and a cube root of volume of $\sim 20 \mathrm{~nm}$ should have a $k_{\mathrm{ARM}} / \mathrm{IRM}$ ratio of about $0.5 \mathrm{~mm} / \mathrm{A}$, whereas the measured value is $1.4 \mathrm{~mm} / \mathrm{A}$. Given the measured median destructive field, the ARM susceptibility measured would be expected for particles with a length of $45 \mathrm{~nm}$ and a length-to-width ratio of 1.3.

[51] The isolated particles in untreated cells of mnm18 produce one of the highest ARM susceptibilities that we have ever observed. With a $k_{\mathrm{ARM}} / \mathrm{IRM}$ of $4.0 \mathrm{~mm} / \mathrm{A}$, they lie among the highest sediment values tabulated by Egli [2004], and above previously measured magnetotactic bacteria [Moskowitz et al., 1993]. Given the similarity of the crystals produced by mnm 18 to those produced by the wild type, the high $k_{\mathrm{ARM}} / \mathrm{IRM}$ is likely due to the absence of magnetostatic interactions. Although they have less effect than three-dimensional interactions, even the linear interactions in wild-type AMB-1 appear to lower lower ARM susceptibility slightly.

[52] At biasing fields below $300 \mu \mathrm{T}$, the ARM/IRM curves of ultrasonicated mnm13 (B2), SDS-treated mnm13 (B3), and SDS-treated mnm18 (C3) are almost identical, whereas above $300 \mu \mathrm{T}$ they diverge, with $\mathrm{B} 2>$ $\mathrm{B} 3>\mathrm{C} 3$. The divergence may reflect the presence of a greater proportion of more strongly interacting particles (which acquire ARM in higher biasing fields) in the more severely treated samples.

\subsection{Low-Temperature Magnetic Properties}

[53] Regardless of treatment, the MV-1 samples have low $\delta_{\mathrm{FC}} / \delta_{\mathrm{ZFC}}$ : the untreated and ultrasonicated samples have $\delta_{\mathrm{FC}} / \delta_{\mathrm{ZFC}}$ of 1.4 , while the SDS-treated MV-1 has $\delta_{\mathrm{FC}} / \delta_{\mathrm{ZFC}}$ of 1.1 (Figure 6e and Table 4). On the basis of the criterion 
Table 4. Low-Temperature Magnetic Parameters

\begin{tabular}{cccc}
\hline Sample & $\delta_{\text {ZFC }}$ & $\delta_{\text {FC }} / \delta_{\text {ZFC }}$ & $f_{\text {LTC }}$ \\
\hline A1 & 0.13 & 2.53 & 0.98 \\
B1 & 0.17 & 1.07 & 0.97 \\
C1 & 0.16 & 2.57 & 0.83 \\
C3 & 0.35 & 1.24 & 0.86 \\
V1 & 0.29 & 1.40 & 0.90 \\
V2a & 0.29 & 1.42 & 0.94 \\
V3a & 0.61 & 1.10 & 0.77 \\
\hline
\end{tabular}

of Moskowitz et al. [1993], $\delta_{\mathrm{FC}} / \delta_{\mathrm{ZFC}}>2$ indicates the presence of chains. The reason why our untreated MV-1 fails this test is unclear, although such low values have previously been observed for some fresh cultures of MV-1 (B. Moskowitz, personal communication, 2006). The low values may be related to the partial chain collapse previously described, but they stand in contrast to FMR data indicating the presence of chains. They are not a product of accidental sample oxidation; the absolute values of $\delta_{\mathrm{FC}}$ and $\delta_{\mathrm{ZFC}}$ are relatively large.

[54] The untreated cells of mutant mnm13 fail the Moskowitz test, with $\delta_{\mathrm{FC}} / \delta_{\mathrm{ZFC}}=1.9$, consistent with the absence of chains in this sample. Inspection of its low-temperature demagnetization curves indicates that the sample's lowtemperature properties are dominated by the unblocking of superparamagnetic grains, in agreement with the smaller grain size observed in the TEM images. In contrast, the untreated cells of mnm18 have $\delta_{\mathrm{FC}} / \delta_{\mathrm{ZFC}}=2.6$, which slightly exceeds the wild-type value of 2.5 even though less than half of the crystals present are in chains. The unexpected result cannot be explained by nonstoichiometry, which would increase $\delta_{\mathrm{FC}} / \delta_{\mathrm{ZFC}}$ while at the same time decreasing $\delta_{\mathrm{FC}}$ and $\delta_{\mathrm{ZFC}}$ [Carter-Stiglitz et al., 2004]. No such drop in $\delta_{\mathrm{FC}}$ and $\delta_{\mathrm{ZFC}}$ is observed. Furthermore, whereas SDS treatment of mnm18 produces an FMR spectrum reflecting the presence of linear particle arrangements, it also causes $\delta_{\mathrm{FC}} / \delta_{\mathrm{ZFC}}$ to drop to 1.2 , comparable to the SDStreated wild type [Kopp et al., 2006]. The elevated $\delta_{\mathrm{FC}} / \delta_{\mathrm{ZFC}}$ ratios of mnm18 may occur because the chain component within the sample has a higher $\delta_{\mathrm{FC}} / \delta_{\mathrm{ZFC}}$ than the wild-type AMB-1 that we measured; previously observed $\delta_{\mathrm{FC}} / \delta_{\mathrm{ZFC}}$ ratios for AMB-1 range as high as 5.9 [Weiss et al., 2004]. Alternatively, the distinctive $\delta_{\mathrm{FC}} / \delta_{\mathrm{ZFC}}$ ratios of magnetotactic bacteria may be due, at least in part, to same factor other than chain structure and nonstoichiometry.

[55] Consistent with prior measurements of wild-type AMB-1 [Kopp et al., 2006], SDS-treated mnm18 exhibits an increase in remanence on cooling through the Verwey temperature, while intact mnm18 exhibits a decrease in remanence. In contrast, both intact and SDS-treated MV-1, like intact AMB-1, exhibit a decrease in remanence upon cooling through the Verwey transition (Figure 6f). We have no explanation for this phenomenon.

\section{Discussion}

[56] As measures of magnetic anisotropy and magnetostatic interaction, FMR parameters should be related to other magnetic properties that are a function of these characteristics. In so far as it possible to fit spectra well and thus obtain an accurate measurement of the anisotropy field of a sample, it is possible to use FMR spectra to estimate the switching field distribution of a sample. Neglecting thermal energy, the median coercive field of a sample is given by $B_{c} \approx \frac{1}{2}\left|B_{a n}\left(1+K_{2} / K_{1}\right)\right|$ [Dunlop and Özdemir, 1997]. A plot of the calculated $B_{c}$ against the median acquisition field of IRM acquisition is shown in Figure $7 \mathrm{a}$.

[57] There is a good correlation between the two parameters, although the estimates derived from the FMR spectra are significantly higher than the measured values. The discrepancy is largely accounted for by the thermal
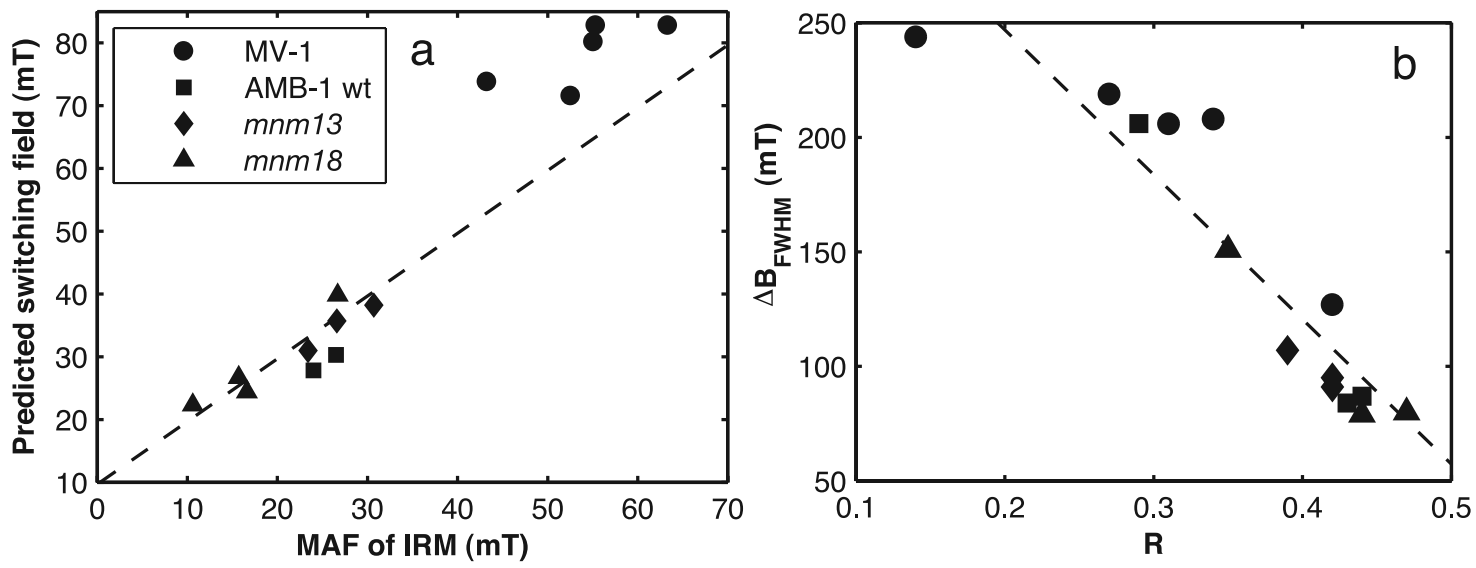

Figure 7. FMR parameters compared to rock magnetic parameters for the samples discussed in this paper. (a) Predicted switching field, determined from the weighted average of $B_{c} \approx \frac{1}{2}\left|B_{a n}\left(1+K_{2} / K_{1}\right)\right|$ of fit components for each sample, plotted against the median acquisition field of IRM. The dashed line represents a line fitted through the points for mnm13 and mnm18 with slope fixed at 1 . The line has a $y$ intercept of $9.7 \mathrm{mT}$ and a coefficient of determination $\mathrm{r}^{2}=0.89$. (b) $\Delta \mathrm{B}_{\mathrm{FWHM}}$ plotted against the Cisowski $R$ parameter, which measures magnetostatic interactions. The dashed line represents a line fitted to all samples and is given by $\Delta \mathrm{B}_{\mathrm{FWHM}}=373 \mathrm{mT}-632 \mathrm{mT} \times \mathrm{R}$. It has a coefficient of determination $r^{2}=0.84$. 


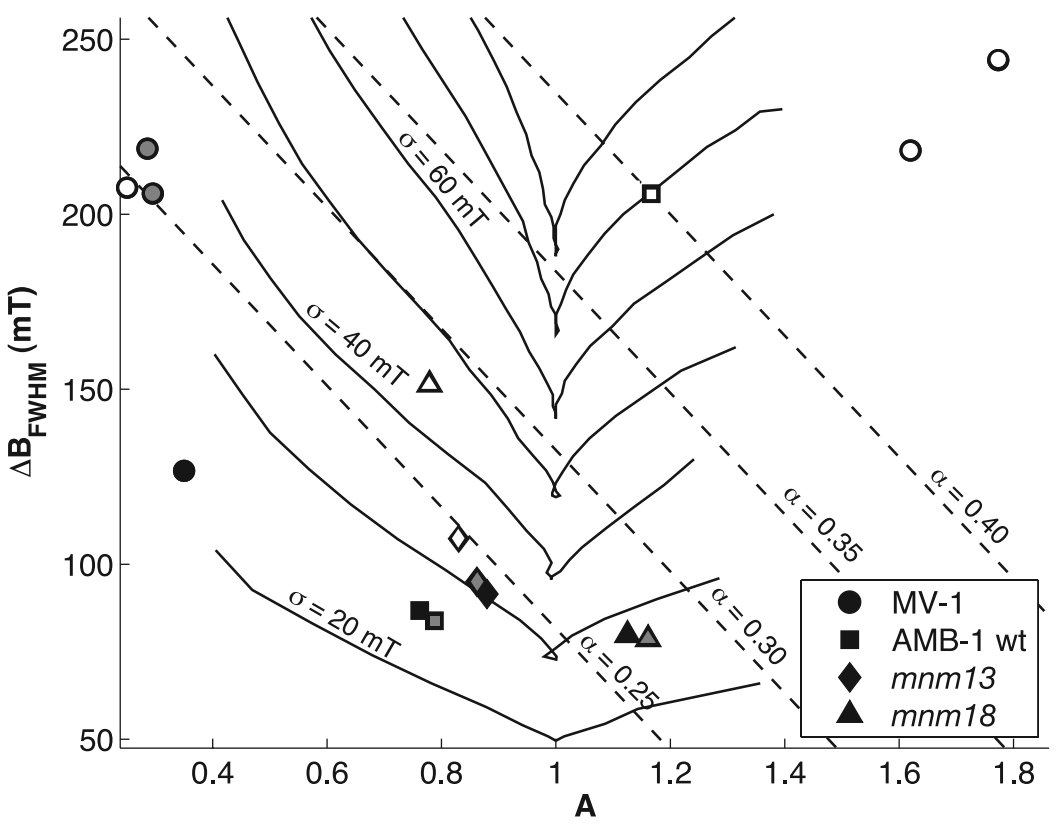

Figure 8. Plot of $\Delta B_{\mathrm{FWHM}}$ against $A$ for the samples discussed in this paper. Solid symbols represent untreated samples, shaded symbols represent ultrasonicated samples, and open samples represented SDStreated samples. The dilution trend for ultrasonicated MV-1 goes slightly from the upper left to the bottom right, while the dilution trend for SDS-treated MV-1 goes from right to left. Dashed lines are contours of constant values of $\alpha$. Solid lines represent simulated spectra with fixed Gaussian line width $\sigma$ and variable $B_{a n}$.

fluctuation field, which for $100 \mathrm{~nm}$ cubes of magnetite at room temperature is approximately $50 \sqrt{B_{c}}$, or about $10 \mathrm{mT}$ for particles with $B_{c}=30 \mathrm{mT}$ [Dunlop and Özdemir, 1997]. Linear regression of the $B_{c}$ values for mnm13 and mnm18, with the slope of the line fixed at 1 because of the expected theoretical relationship between $B_{c}$ and MAF, yields the line $B_{c}=\mathrm{MAF}+9.7 \mathrm{mT}$, with a coefficient of determination $r^{2}=0.89$. Removing the constraint on the slope does not significantly improve the fit. The y intercept thus calculated is in agreement with the expected thermal fluctuation field.

[58] Cells of mutant AMB-1 and intact cells of wild-type AMB-1 have $B_{c}$ close to those predicted from the regression line, but SDS-treated cells of AMB-1 and all MV-1 samples fall well off the line. This difference may be due to a combination of imperfect fitting of the FMR spectra and the presence of additional factors not treated in the simple physical model used to predict $B_{c}$.

[59] There is no single parameter that perfectly reflects interaction field strength [Dunlop et al., 1990], but the crossover $R$ value of Cisowski [1981] is commonly used. The strength of three-dimensional magnetostatic interactions affects two parameters employed in modeling FMR spectra: the anisotropy field $B_{a n}$ and the Gaussian line width $\sigma$. Local anisotropy in magnetostatic interactions, such as that which occurs on the surface of a clump of particles, alters the anisotropy field, while the heterogeneity of local magnetic environments produced by interactions results in an increase in Gaussian line width. Other factors also contribute to both these terms, however, so neither provides a good measure of interaction field strength. The empirical line width parameter $\Delta B_{\mathrm{FWHM}}$ appears to provide a better measure, as it correlates reasonably well with the Cisowski
$R$ parameter (Figure 7b). Linear regression yields the relationship $\Delta B_{\mathrm{FWHM}}=373 \mathrm{mT}-632 \mathrm{mT} \times R$, with a coefficient of determination $r^{2}=0.84$. When present, strong three-dimensional interactions overwhelm other factors controlling $\Delta B_{\mathrm{FWHM}}$, such as single-particle anisotropy and linear interactions.

[60] The bacterial samples measured in this work continue to support the use of the empirical discriminant factor $\alpha$ [Kopp et al., 2006] to distinguish biogenic magnetite chains (Figure 8). Of all the intact cells of magnetotactic bacteria we measured, only those of the mutant mnm18 have $\alpha>0.24$. This exception arises because mnm 18 has $A>1$ and, while $\alpha$ serves as a proxy for Gaussian line width $\sigma$ when $\sigma$ is around $30 \mathrm{mT}$ and $A<1$, it does not when $A>1$, as can be seen from the $\alpha$ contours plotted on Figure 8. As can be seen from the contours on Figure 8, mnm 18 falls within the domain of intact magnetotactic bacterial cells when $\sigma$ values of synthetic spectra are used to delineate boundaries.

[61] Ultrasonication in general results in a slight increase in $\alpha$, which confirms prior results [Kopp et al., 2006]. SDS treatments of the wild-type cells of both MV-1 and AMB-1 result in drastic shifts in $\alpha$ as highly interacting clumps come to dominate the sample. The increase in $\alpha$ that occurs with SDS treatment of cells of the AMB-1 mutants, in which the magnetite is more dilute, is present but subtle. SDS-treated cells of both wild-type strains, when diluted by mixing for $5 \mathrm{~min}$, experience a significant reduction in $\alpha$ to values characteristic of the domain previously identified as being the magnetofossil domain, namely $\alpha<0.30$ [Kopp et al., 2006].

[62] In agreement with Kopp et al. [2006] and Weiss et al. [2004], these data support the use of ferromagnetic reso- 
nance spectroscopy as a technique for identifying potential magnetofossils in the sedimentary record. Because it can provide a rapid way of estimating the biogenic contribution to sedimentary magnetism, FMR has the potential to be a highly useful tool for environmental magnetism and magnetic paleobiology.

\section{Conclusion}

[63] We have generated mutant strains of magnetotactic bacteria that allow us to start to untangle the contributions of chain arrangement and particle elongation to the ferromagnetic resonance and rock magnetic properties of magnetotactic bacteria. The four strains we have analyzed represent all four possible combinations of chain and solitary particles, and elongate and equidimensional particles. In addition, the SDS-treated cells of mnm18 allow us to investigate the changes that occur as solitary equidimensional particles assemble into linear structures. Our findings indicate that ferromagnetic resonance spectroscopy provides an effective technique for estimating the switching field distribution and interaction effects within a sample and continue to support the use of ferromagnetic resonance spectroscopy as a way of identifying magnetotactic bacteria and magnetofossils. Since it takes only a few minutes to acquire a FMR spectrum, which is significantly faster than most rock magnetic techniques being used for similar purposes, we hope that our work will spur the broader adoption of ferromagnetic resonance spectroscopy by the rock magnetic community.

\section{Notation}

FMR: Empirical Parameters

$A$ asymmetry ratio $=\Delta B_{\text {high }} /$ $\Delta B_{\text {low. }}$

$B_{\text {eff }}$ applied field at peak of integrated absorption spectrum, mT.

$\triangle B_{\mathrm{FWHM}}$ full width at half maximum,

$\Delta B_{\text {high }}\left(\Delta B_{\text {low }}\right) \quad \begin{array}{ll}\Delta B_{\text {high }}+\Delta B_{\text {low. }} \\ \text { half width at half maximum }\end{array}$ of integrated spectrum on high-field (low-field) side of peak, $\mathrm{mT}$.

$g_{\text {eff }} g$ value at absorption peak, $h \nu / \beta B_{\text {eff. }}$

$\alpha$ empirical discriminant factor, $0.17 A+9.8 \times 10^{-4} \mathrm{mT}^{-1}$ $\Delta B_{\text {FWHM. }}$

FMR: Empirical Parameters

$B_{a n}$ effective anisotropy field: $2 K_{1} / M$ for magnetocrystalline anisotropy, $\mu_{0} M_{s} \Delta N$ for shape anisotropy.

$g$ true spectroscopic $g$-factor (equivalent to $g_{\text {eff }}$ when $\left.B_{a n}=0\right)$.

$K_{2} / K_{1} \quad$ ratio of second-order and firstorder anisotropy constants.

$\sigma$ standard deviation of Gaussian broadening function.

\author{
Rock Magnetic Parameters \\ $H_{c r}$ coercivity of remanence, de- \\ termined here from intersec- \\ tion point of IRM acquisition \\ and demagnetization curves, \\ $\mathrm{mT}$. \\ $f_{\text {LTC }}$ fraction of room temperature \\ SIRM retained after cycling \\ to low temperature and back. \\ $k_{\mathrm{ARM}} / \mathrm{IRM}$ ARM susceptibility normal- \\ ized to IRM (measured here \\ with $0.1 \mathrm{mT}$ ARM biasing \\ field, $100 \mathrm{mT}$ ARM alternat- \\ ing field, and $100 \mathrm{mT}$ IRM \\ pulse field), $\mathrm{mm} / \mathrm{A}$. \\ MAF (MDF) median acquisition (destruc- \\ tive) fields, at which half of \\ a total remanence is acquired \\ (destroyed), $\mathrm{mT}$. \\ $R$ Cisowski $\mathrm{R}$ parameter, re- \\ flecting magnetostatic inter- \\ actions: fraction of IRM \\ remaining at $H_{c r}$. \\ $\delta_{\mathrm{FC}}\left(\delta_{\mathrm{ZFC}}\right) \quad\left(J_{80 \mathrm{~K}}-J_{150 \mathrm{~K}}\right) / J_{80 \mathrm{~K}}$ for field- \\ cooled (zero-field-cooled) \\ low-temperature SIRM ther- \\ mal demagnetization curves.
}

[64] Acknowledgments. We thank Angelo Di Bilio for assistance with the EPR spectrometer, Arash Komeili for assistance with the mutagenesis, and Mike Jackson, David Griscom, and an anonymous reviewer for helpful comments. The Beckman Institute provided support for the use of the MPMS. R.E.K., J.L.K., and C.Z.N. would like to thank the Agouron Institute, the Moore Foundation, and the NASA Astrobiology Science and Technology Instrument Development program for support. A.K. was partially supported by funds from a New Energy and Industrial Technology Development Organization fellowship. D.A.B. was supported by U.S National Science Foundation grant EAR-0311950. B.P.W. thanks the NASA Mars Fundamental Research and NSF Geophysics Programs.

\section{References}

Carter-Stiglitz, B., B. Moskowitz, and M. Jackson (2004), More on the lowtemperature magnetism of stable single domain magnetite: Reversibility and non-stoichiometry, Geophys. Res. Lett., 31, L06606, doi:10.1029/ 2003 GL019155.

Chang, S. B. R., and J. L. Kirschvink (1989), Magnetofossils, the magnetization of sediments, and the evolution of magnetite biomineralization, Annu. Rev. Earth Planet. Sci., 17, 169-195.

Chang, S. B. R., et al. (1989), Biogenic magnetite in stromatolites. 2. Occurrence in ancient sedimentary environments, Precambrian Res., $43,305-315$.

Cisowski, S. (1981), Interacting vs. non-interacting single-domain behavior in natural and synthetic samples, Phys. Earth Planet. Inter, 26, 56-62.

Dean, A. J., and D. A. Bazylinski (1999), Genome analysis of several marine, magnetotactic bacterial strains by pulsed-field gel electrophoresis, Curr. Microbiol., 39, 219-225.

Devouard, B., et al. (1998), Magnetite from magnetotactic bacteria: Size distributions and twinning, Am. Mineral., 83, 1387-1398.

Dunlop, D. J., and Ö. Özdemir (1997), Rock Magnetism: Fundamentals and Frontiers, 573 pp., Cambridge University Press, New York.

Dunlop, D. J., et al. (1990), Preisach diagrams and anhysteresis: Do they measure interactions, Phys. Earth Planet. Inter., 65, 62-77.

Egli, R. (2003), Analysis of the field dependence of remanent magnetization curves, J. Geophys. Res., 108(B2), 2081, doi:10.1029/ 2002JB002023.

Egli, R. (2004), Characterization of individual rock magnetic components by analysis of remanence curves, 1 . Unmixing natural sediments, Studia Geophys. Geod., 48, 391-446.

Egli, R., and W. Lowrie (2002), Anhysteretic remanent magnetization of fine magnetic particles, J. Geophys. Res., 107(B10), 2209, doi:10.1029/ 2001JB000671. 
Griscom, D. L. (1974), Ferromagnetic resonance spectra of lunar fines: Some implications of line shape analysis, Geochim. Cosmochim. Acta, $38,1509-1519$.

Griscom, D. L. (1981), Ferromagnetic resonance condition and powder pattern analysis for dilute, spherical, single-domain particles of cubic crystal structure, J. Magn. Reson., 45, 81-87.

Griscom, D. L., et al. (1988), Ferromagnetic-resonance studies of ironimplanted silica, Nucl. Instrum. Methods Phys. Res., Sect. B, 32, $272-$ 278.

Hanzlik, M., et al. (2002), Pulsed-field-remanence measurements on individual magnetotactic bacteria, J. Magn. Magn. Mater., 248, 258-267.

Kąkol, Z., and J. M. Honig (1989), Influence of deviations from ideal stoichiometry on the anisotropy parameters of magnetite $\mathrm{Fe}_{3(1-\delta)} \mathrm{O}_{4}$, Phys. Rev. B, 40, 9090-9097.

Kirschvink, J. L., and S. B. R. Chang (1984), Ultrafine-grained magnetite in deep-sea sediments: Possible bacterial magnetofossils, Geology, 12, $559-562$.

Kirschvink, J. L., and H. A. Lowenstam (1979), Mineralization and magnetization of chiton teeth: Paleomagnetic, Sedimentologic, and biologic implications of organic magnetite, Earth Planet. Sci. Lett., 44, 193-204.

Kittel, C. (1948), On the theory of ferromagnetic resonance absorption, Phys. Rev., 73, 155-161.

Kobayashi, A., et al. (2006), Experimental observation of magnetosome chain collapse in magnetotactic bacteria: Sedimentological, paleomagnetic, and evolutionary implications, Earth Planet. Sci. Lett., 245, 538-550.

Komeili, A., et al. (2004), Magnetosome vesicles are present before magnetite formation, and MamA is required for their activation, Proc. Natl. Acad. Sci. U.S.A., 101, 3839-3844.

Komeili, A., et al. (2006), Magnetosomes are cell membrane invaginations organized by the actin-like protein MamK, Science, 311, 242-245.

Kopp, R. E., et al. (2006), Chains, clumps, and strings: Magnetofossil taphonomy with ferromagnetic resonance spectroscopy, Earth Planet. Sci. Lett., 10-25.

Moskowitz, B. M., et al. (1988), Magnetic properties of magnetotactic bacteria, J. Magn. Magn. Mater., 73, 273-288.

Moskowitz, B. M., et al. (1993), Rock magnetic criteria for the detection of biogenic magnetite, Earth Planet. Sci. Lett., 120, 283-300.
Muxworthy, A. R., and E. McClellan (2000), Review of the lowtemperature magnetic properties of magnetite from a rock magnetic perspective, Geophys. J. Int., 140, 101-114.

Osborn, J. A. (1945), Demagnetizing factors of the general ellipsoid, Phys. Rev., 67, 351-357.

Pan, Y. X., et al. (2005), Rock magnetic properties of uncultured magnetotactic bacteria, Earth Planet. Sci. Lett., 237, 311-325.

Penninga, I., et al. (1995), Remanence measurements on individual magnetotactic bacteria using a pulsed magnetic-field, J. Magn. Magn. Mater, 149, 279-286.

Scheffel, A., et al. (2006), An acidic protein aligns magnetosomes along a filamentous structure in magnetotactic bacteria, Nature, 440, 110-114.

Schlömann, E. (1958), Ferromagnetic resonance in polycrystal ferrites with large anisotropy: General theory and application to cubic materials with a negative anisotropy constant, J. Phys. Chem. Solids, 6, 257-266.

Smit, J., and H. G. Beljers (1955), Ferromagnetic resonance absorption in $\mathrm{BaFe}_{2} \mathrm{O}_{19}$, a highly anisotropic crystal, Philips Res. Rep., 10, 113-130. Thomas-Keprta, K. L. (2000), Elongated prismatic magnetite crystals in ALH84001 carbonate globules: Potential Martian magnetofossils, Geochim. Cosmochim. Acta, 64, 4049-4081.

Weiss, B. P., et al. (2004), Ferromagnetic resonance and low temperature magnetic tests for biogenic magnetite, Earth Planet. Sci. Lett., 224, $73-89$.

D. A. Bazylinski, Department of Biochemistry, Biophysics, and Molecular Biology, Iowa State University, Ames, IA 50011, USA.

J. L. Kirschvink, R. E. Kopp, and C. Z. Nash, Division of Geological and Planetary Sciences, California Institute of Technology, 170-25, Pasadena, CA 91125, USA. (rkopp@caltech.edu)

A. Kobayashi, Photonics Research Institute, National Institute of Advanced Industrial Science and Technology, 1-8-31 Midorigaoka, Ikeda, Osaka 563-8577, Japan.

B. P. Weiss, Department of Earth, Atmospheric, and Planetary Sciences, Massachusetts Institute of Technology, Cambridge, MA 02139, USA. 


\title{
Chains, clumps, and strings: Magnetofossil taphonomy with ferromagnetic resonance spectroscopy
}

\author{
Robert E. Kopp ${ }^{\text {a,* }}$, Benjamin P. Weiss ${ }^{b}$, Adam C. Maloof ${ }^{b, 1}$, Hojotollah Vali ${ }^{\text {c,d }}$, \\ Cody Z. Nash ${ }^{\text {a }}$, Joseph L. Kirschvink ${ }^{\text {a }}$ \\ a Division of Geological and Planetary Sciences, California Institute of Technology, Pasadena, CA 91125, USA \\ ${ }^{\mathrm{b}}$ Department of Earth, Atmospheric, and Planetary Sciences, Massachusetts Institute of Technology, Cambridge, MA 02139, USA \\ ${ }^{\mathrm{c}}$ Department of Anatomy and Cell Biology and Facility for Electron Microscopy Research, McGill University, Montréal, QC, Canada H3A 2B2 \\ d Department of Earth and Planetary Sciences, McGill University, Montréal, QC, Canada H3A $2 A 7$
}

Received 15 February 2006; received in revised form 26 April 2006; accepted 1 May 2006

Editor: S. King

\begin{abstract}
Magnetotactic bacteria produce intracellular crystals of magnetite or greigite, the properties of which have been shaped by evolution to maximize the magnetic moment per atom of iron. Intracellular bacterial magnetite therefore possesses traits amenable to detection by physical techniques: typically, narrow size and shape distributions, single-domain size and arrangement in linear chains, and often crystal elongation. Past strategies for searching for bacterial magnetofossils using physical techniques have focused on identifying samples containing significant amounts of single domain magnetite or with narrow coercivity distributions. Searching for additional of traits would, however, increase the likelihood that candidate magnetofossils are truly of biological origin. Ferromagnetic resonance spectroscopy (FMR) is in theory capable of detecting the distinctive magnetic anisotropy produced by chain arrangement and crystal elongation. Here we present analyses of intact and lysed magnetotactic bacteria, dilutions of synthetic magnetite, and sedimentary samples of modern carbonates from the Great Bahama Bank, OligoceneMiocene deep-sea muds from the South Atlantic, and Pleistocene lacustrine deposits from Mono Basin, California. We demonstrate that FMR can distinguish between intact bacterial magnetite chains, collapsed chains, and linear strings of magnetite formed by physical processes. We also show that sediments in which the magnetization is likely carried by bacterial magnetite have FMR spectra resembling those of intact or altered bacterial magnetite chains.
\end{abstract}

(C) 2006 Elsevier B.V. All rights reserved.

Keywords: magnetotactic bacteria; biogenic magnetite; ferromagnetic resonance; magnetofossils

\footnotetext{
* Corresponding author. Division of Geological and Planetary Sciences, MC 170-25, California Institute of Technology, Pasadena, CA 91125, USA. Tel.: +1 626395 2949; fax: +1 6265680935.

E-mail addresses: rkopp@caltech.edu (R.E. Kopp), bpweiss@mit.edu (B.P. Weiss), maloofa@mit.edu (A.C. Maloof), vali@eps.mcgill.ca (H. Vali), cody@caltech.edu (C.Z. Nash), kirschvink@caltech.edu (J.L. Kirschvink).

${ }^{1}$ Present address: Department of Geosciences, Princeton University, Princeton, NJ 08544, USA.
}

\section{Introduction}

Magnetotactic bacteria are a polyphyletic group of organisms that, uniquely among the bacteria, engage in biologically organized mineralization. They precipitate intracellular crystals of ferrimagnetic iron minerals, either magnetite $\left(\mathrm{Fe}_{3} \mathrm{O}_{4}\right)$ or greigite $\left(\mathrm{Fe}_{3} \mathrm{~S}_{4}\right)$, within membrane-bound organelles called magnetosomes. 
Although magnetosomes likely have multiple functions $[1,2]$, one major role is the passive alignment of bacterial cells with the geomagnetic field. Magnetotactic bacteria tend to live in regions with well-defined redox gradients. Everywhere except on Earth's geomagnetic equator, the geomagnetic field has a vertical component; by swimming along the field lines, the bacteria reduce a three-dimensional search for optimal chemical conditions to a one-dimensional search [3-5].

Because natural selection can lead magnetotactic lineages to maximize their magnetic sensitivity for the amount of iron used, magnetite crystals produced by magnetotactic bacteria have a number of distinctive traits that facilitate their identification as magnetofossils in sedimentary deposits [6]. Not all magnetotactic organisms produce magnetite with all of these traits, and abiotic processes can produce crystals with some of them, but the greater the number of traits present, the higher the degree of confidence with which one can identify magnetite as biogenic. The following traits have been identified so far. Magnetite from magnetotactic bacteria is generally chemically pure and lacking in crystallographic defects (but see the relatively rare anomalies described by [7]). It almost always has a narrow size and shape distribution, is stably or metastably single domain, and is arranged in chains of particles. The crystals also tend to have unusual crystal morphologies that increase the single domain stability field $[8,9]$ : sometimes elongated along the magnetocrystalline easy axis, and often with truncated crystal edges.

Because magnetotactic bacteria prefer specific chemical environments, their fossils convey information about paleoclimate and paleoecology [10,11]. Magnetofossils also have a major advantage over other bacterial fossils: because the fossils are magnetic, samples likely to contain magnetofossils can potentially be identified using physical techniques rather than timeconsuming electron microscopy surveys. Nevertheless, the pre-Quaternary fossil record of magnetotactic bacteria is sparse. The oldest samples with chains of single domain particles with distinctively biological morphologies are Cretaceous chalks from England [12], although a few putative Precambrian magnetofossils have also been identified $[4,13]$.

Previous attempts to search for magnetofossils in preQuaternary sediments with physical techniques have relied on the use of basic rock magnetic procedures designed to identify single domain magnetite [e.g. 13]. However, single domain magnetite may be precipitated or concentrated by abiotic processes and is not unique to magnetofossils. Finding additional characteristic traits would greatly strengthen identifications. New techniques for unmixing coercivity spectra [14,15] allow determination of the degree of variation within populations of magnetic particles, and thus allow detection of the narrow particle size and shape distributions characteristic of magnetotactic bacteria and magnetofossils. Acquiring high-resolution coercivity spectra is a time-intensive process, however. Although the process can be abbreviated for examining stratigraphic variation within a section where the end-member components have been identified [11], its time requirements may limit its utility in surveying the deep-time magnetofossil record.

The magnetofossil chain structure and magnetosome crystal elongation should exert distinctive influences on microscale magnetic anisotropy and should therefore be detectable using physical techniques. For instance, Moskowitz et al. [16] proposed that the relative magnitude of demagnetization upon warming across magnetite's Verwey transition under field cooled and zero-field cooled conditions is an indicator of chain structure. While their test did successfully distinguish fresh bacterial magnetite from other sources of magnetite, it is highly susceptible to crystal oxidation and thus to false negatives. In application to sediments, it also needs to be corrected for mixing of materials [15].

Isolated, equidimensional magnetite particles are dominated by negative cubic magnetocrystalline anisotropy, such that particle energy is highest when magnetization is aligned along the principal axes of the cubic crystal system and lowest when magnetization is aligned with the family of [111] crystal axes. Positive uniaxial shape anisotropy related to crystal elongation and particle organization in linear chains, in contrast, minimizes magnetostatic energy when the magnetization is aligned parallel to the crystal or chain elongation axis and maximizes magnetostatic energy when the magnetization is orthogonal to the crystal or chain elongation axis. Lowering the magnetostatic energy is equivalent to applying a field along the direction of magnetization, while increasing the energy of a state is equivalent to applying a field against the magnetization. Thus, a physical technique capable of assessing the effective field felt by a particle should be sensitive to chain structure and elongation. Ferromagnetic resonance spectroscopy (FMR) is such a technique [17-20].

FMR is a form of electron spin resonance, also known as electron paramagnetic resonance (EPR) [21]. FMR is based on the Zeeman effect, which causes the energy of an electron with spin aligned with a magnetic field to be lower than that of an electron with spin aligned against the field. The energy splitting is given by 
$\Delta E=g \beta B$, where $g$ is the spectroscopic $\mathrm{g}$-factor, $\beta$ is the Bohr magneton $\left(9.37 \times 10^{-24} \mathrm{Am}^{2}\right)$, and $B$ is the field felt by the electron. The spectroscopic g-factor is 2.0023 for a free electron and 2.12 for an electron in magnetite $[21,22]$. The electron can therefore absorb a photon with energy $\mathrm{h} v=g \beta B$.

In a conventional EPR spectrometer, a sample is placed in a resonating cavity situated inside an electromagnet. A microwave radiation source with limited frequency adjustability (typically X-band, $\sim 9.0-9.8 \mathrm{GHz}$ ) generates photons. A detector measures the first derivative of absorption as the electromagnet sweeps the magnetic field strength across a range of values [21]. For a single crystal, the resonant field is shifted to lower values when magnetization is aligned along a magnetically easy direction (e.g., along the [111] crystal axis of magnetite, or along the elongation axis of an elongate crystal or chain) and to higher values when the magnetization is aligned along a hard direction.

Most samples of geological interest are not single crystals, but are composed of particles in a variety of arrangements and orientations with respect to the applied magnetic field. The spectrum of a powder of magnetostatically non-interacting, spherical (isotropic) particles is simply a broadened form of the single crystal spectrum (Fig. 1A), but for powders of anisotropic particles, the spectrum not only broadens but also develops asymmetry (Fig. 1B and E). Consider the case of a powder of elongate magnetite crystals, with elongation axes distributed uniformly in all directions (Fig. 1C). From simple geometrical considerations, there will be a small number of particles with elongation axes closely aligned with the direction of the applied field, and many more with elongation axes aligned close to the plane perpendicular to the applied field. Those with elongation axes aligned with the applied field will absorb at lower fields than would isotropic particles, while those with elongation axes in the normal plane will absorb at higher fields. Thus, compared to the spectrum of a powder of isotropic particles, the peak absorption will be shifted to higher field values (i.e., lower effective g-factor) but will have a tail asymmetrically extended in the low field direction. Conversely, a powder of particles with negative anisotropy will have increased effective g-factors and high field extended asymmetry (Fig. 1B and D).

As can be seen in Fig. $1 \mathrm{C}$ and $\mathrm{D}$, the derivative spectrum of a powder of particles with uniaxial anisotropy has two peaks on the asymmetrically extended side. The one closer to the zero crossing is associated with the rise to the peak of absorption, while the one farther from the zero crossing is associated with

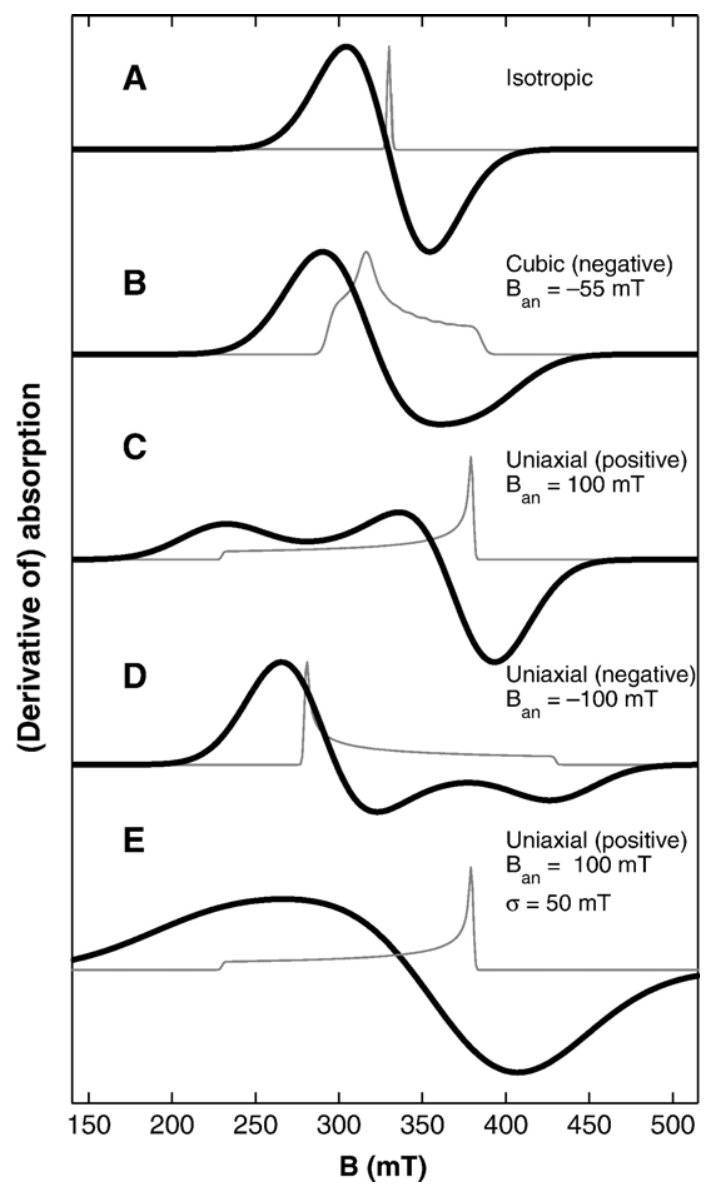

Fig. 1. Synthetic FMR spectra. Generated with $g=2.12$, and (A) no magnetic anisotropy, (B) cubic $B_{\mathrm{an}}=-55 \mathrm{mT}$, as expected for noninteracting cubic magnetite, (C) uniaxial $B_{\text {an }}=100 \mathrm{mT}$, (D) uniaxial $B_{\text {an }}=-100 \mathrm{mT}$, and (E) uniaxial $B_{\mathrm{an}}=100 \mathrm{mT}$. For (A-D), $\sigma=25 \mathrm{mT}$; for (E), $\sigma=50 \mathrm{mT}$. Thick lines show derivative spectra and thin lines show integrated absorption spectra with Gaussian broadening lowered to $\sigma=3 \mathrm{mT}$. Sharp spectra with positive uniaxial anisotropy, as in (C), have two local maxima on the low field side, while sharp spectra with negative uniaxial anisotropy, as in (D), have two local minima on the high field side; these features can be obscured by spectral broadening, as seen in comparison of (C) and (E).

the transition from zero absorption to positive absorption. Samples with more complex anisotropies may have additional peaks. These differences, however, can be obscured by factors that symmetrically broaden linewidth, such as magnetostatic interactions and sample heterogeneity (Fig. 1E).

Weiss et al. [20] found that bacterial magnetite chains have FMR spectra distinct from abiotic magnetite. They postulated that the unique spectra of magnetosome chains result from the positive magnetic anisotropy of the chain structure (Fig. 1C), which contrasts with the negative cubic magnetocrystalline anisotropy of roomtemperature magnetite (Fig. 1B). They demonstrated, 
through measurements of laboratory cultures, that intact magnetotactic bacteria have spectra with low-field extended asymmetry, as well as effective g-factors shifted below the standard magnetite value of 2.12 and two local maxima. Weiss et al. [20] also showed that lysed (broken) chains do not have the distinct FMR signature of intact chains, which suggests that the chain structure plays a major role in the FMR signature. However, because the crystals in the lysed chains in their experiments were clumped and highly magnetostatically interacting, Weiss et al. were unable to measure the FMR spectra of isolated magnetosomes and thus to demonstrate that the characteristic FMR spectra of magnetosome chains arises from the chain structure rather than from some trait of the individual crystals.

In this paper, we investigate the effects of chain structure and crystal elongation on FMR spectra and the extent to which these features can be mimicked by process other than the growth of chains of magnetite within magnetotactic bacteria. We examined intact and disrupted chains of magnetite from Magnetospirillum magneticum strain AMB-1, as well as dilutions of magnetite from lysed AMB-1 and of abiogenic magnetite strung out into linear features. We also examined sediments from a modern carbonate platform in the Bahamas, Oligocene-Miocene clays and calcareous oozes from the Atlantic Ocean, and lacustrine silts and muds from Mono Basin, California, in an attempt to determine whether these features corresponded to those observed in nature.

\section{Samples}

\subsection{Bacterial cultures}

For the lysis experiments, $2 \mathrm{~L}$ of $M$. magneticum strain AMB-1 (ATCC strain 700264) was grown to early stationary phase, at $\sim 10^{8}$ cells/ $\mathrm{mL}$, using standard culture conditions [23] and then spun down into three subsamples, which were resuspended in $5 \mathrm{~mL}$ Tris buffer. Five microliters of $\beta$-mercaptoethanol was added to subsample A3. The samples were frozen at $-20{ }^{\circ} \mathrm{C}$ and then thawed at $5{ }^{\circ} \mathrm{C}$. Then, $270 \mathrm{mg}$ of sodium dodecyl sulfate (SDS) was added as a detergent to A3, which was incubated at room temperature for $10 \mathrm{~min}$. A2 and A3 were subjected to ultrasonication with a Fischer Scientific Sonic Dismembrator 550 for $6 \mathrm{~min}$, with pulses of $0.5 \mathrm{~s}$ alternated with pauses of $0.5 \mathrm{~s}$.

Next, the samples were spun down, frozen, and freeze-dried. Half of A3 was diluted to $\sim 0.1 \%$ in powdered sucrose by grinding in an agate mortar and pestle, first for $1 \mathrm{~min}$ (to form subsample A3b) and then for $5 \mathrm{~min}$ (to form subsample A3c). Halves of subsamples A1 and A2 were similarly diluted by grinding for 5 min to form subsamples A1b and A2b.

Based on rock magnetic data and transmission electron microscope images acquired in previous studies from the related magnetotactic bacterium M. magnetotacticum strain MS-1 [24,25], we expect that ultrasonicated AMB-1 contains chains of magnetite crystals liberated from cells but remains bound by magnetosome membranes. The crystals are likely strung together in composite chains formed by linkage of individual chains from different cells, with some of the chains closed to form loops (see Fig. 1 of [25]). Closed loops represent the minimum energy configuration for four or more equidimensional magnetite crystals [26].

SDS treatment, in contrast, destroys both cytoplasmic and magnetosome membranes. As a consequence, the crystals in SDS-treated samples collapse into agglomerates with strong three-dimensional magnetostatic interactions. We predict that, when progressively dispersed to lower density, the crystals minimize their energy by transitioning to increasingly linear "stringy" arrangements, with magnetic dipoles oriented in a headto-tail fashion [26].

\subsection{Synthetic magnetite dilutions}

TMB-100 is a synthetic magnetite powder produced by the Toda Industrial Company. The powder contains predominantly equidimensional, octahedral crystals of 80-200 nm diameter. In concentrated form, the crystals form magnetostatically interacting masses with diameters of several microns [20].

Aliquots of TMB-100 were mixed in a tube with powdered sucrose. Each mixture was shaken to disperse the magnetite through the sucrose. Undispersed accumulations of magnetite were removed with a small $\mathrm{NdFeB}$ magnet. The final mass ratios of magnetite to sucrose were approximately 6\%, $6000 \mathrm{ppm}$, and $400 \mathrm{ppm}$ (for samples T1, T2, and T3 respectively). Subsamples of each mixture were powdered with an agate mortar and pestle for $1 \mathrm{~min}$ and then for $4 \mathrm{~min}$.

\subsection{Sedimentary samples}

Holocene-Recent peritidal carbonate mud samples were collected from a $210 \mathrm{~cm}$ core $\left(\mathrm{C} 51: \mathrm{N} 25^{\circ} 1.35^{\prime} \mathrm{W}\right.$ $78^{\circ} 9.9^{\prime}$ in the WGS84 datum) through a levee crest in the Triple Goose Creek region of Andros Island, the Bahamas. The core was extracted using a Livingstone piston corer provided to us by the Limnological Research Center, University of Minnesota. The core 
was separated into halves and sediment was collected in non-magnetic plastic boxes from interior sediment that had not been in contact with any metal objects. The upper $3 \mathrm{~cm}$ of the core is characterized by active microbial mats, containing the morphologically-identified filamentous cyanobacteria Schizothrix [27]. The active microbial mat is underlain by $29 \mathrm{~cm}$ of tan, faintly-laminated mud, which is in turn underlain by $178 \mathrm{~cm}$ of grey, bioturbated carbonate mud, with variable concentrations of $<1 \mathrm{~cm}$ long cerithid gastropod shells.

Samples of Oligocene-Miocene deep-sea clay and calcareous nanofossil ooze from the southern Atlantic Ocean were taken from the Deep Sea Drilling Project site 522, cores 12-20 (44-80 mbsf) [28]. The magnetic mineralogy of these samples, which is dominated by biogenic magnetic, was described by Vali and Kirschvink [29]. Samples were provided by the Ocean Drilling Program (ODP), which is sponsored by the U. S. National Science Foundation (NSF) and participating countries under management of Joint Oceanographic Institutions (JOI), Inc.

Samples of the Pleistocene Wilson Creek Formation of Mono Basin, California [30], were collected with permission of the Forest Service in May 2005 at the formation's type section northwest of Mono Lake (N $38^{\circ} 1.3^{\prime} \mathrm{W} 119^{\circ} 7.5^{\prime}$ in the WGS 84 datum). Aside from a few ash layers and sand lenses, the sediments of the Wilson Creek Formation are dominantly laminated (unbioturbated) muds and silts derived from the crystalline rocks of the Sierra Nevada to the west.

\section{Methods}

\subsection{Rock magnetic measurements}

Room temperature magnetic remanence experiments were performed using a $2 \mathrm{G}$ Enterprises SQUID magnetometer housed in a magnetically-shielded room. Anhysteretic remanent magnetization (ARM) was acquired in a $100 \mathrm{mT}$ alternating field and a DC biasing field progressively varied in steps from 0 to $1 \mathrm{mT}$ and was then removed by progressive alternating field demagnetization (AF) up to $160 \mathrm{mT}$. Isothermal remanent magnetization (IRM) at $100 \mathrm{mT}$ was then acquired and removed by AF.

The ARM susceptibility of non-interacting single domain particles is a function of temperature, particle moment, and particle switching field [31]. Threedimensional interparticle magnetostatic interactions significantly reduce ARM susceptibility [32], so comparison of the ARM susceptibility of similar particles in different arrangements reflects changes in particle arrangement. We report ARM susceptibility as $k_{\mathrm{ARM}} / \mathrm{IRM}$, the ARM acquired per $\mathrm{A} / \mathrm{m}^{2}$ with a biasing field of $0.1 \mathrm{mT}(79.6 \mathrm{~A} / \mathrm{m})$ and an alternating field of $100 \mathrm{mT}$, normalized to the IRM acquired in a field of $100 \mathrm{mT}$. Typical freeze-dried magnetotactic bacteria (weakly interacting) have $k_{\mathrm{ARM}} / \mathrm{IRM}=2.5 \mathrm{~mm} / \mathrm{A}$, while a typical chiton tooth (strongly interacting) has $k_{\mathrm{ARM}} / \mathrm{IRM}=0.09 \mathrm{~mm} / \mathrm{A}$.

Low-temperature experiments were performed using a Quantum Design Magnetic Properties Measurement System in the Molecular Materials Resource Center of the Beckman Institute at Caltech. Field cooled and zero-field cooled curves were acquired by cooling the sample either in a $3 \mathrm{~T}$ field or in zero field to $5 \mathrm{~K}$, respectively, followed by pulsing with a $3 \mathrm{~T}$ field and then measuring the remanence magnetization during warming to room temperature in zero field. The combination of field cooled and zero field cooled measurements constitute the Moskowitz test $[16,20]$. The sample was then cycled through low-temperature by pulsing it with a $3 \mathrm{~T}$ field at room temperature and then measuring the remanent magnetization as the sample was cooled to $10 \mathrm{~K}$ and then warmed to room temperature.

The results of the low-temperature experiments are reported as the parameters $\delta_{\mathrm{ZFC}}, \delta_{\mathrm{FC}}$, and $f_{\mathrm{LTC}}$. The parameters $\delta=\left(J_{80 \mathrm{~K}}-J_{150 \mathrm{~K}}\right) / J_{80 \mathrm{~K}}$ assessed for the zerofield cooled and field cooled curves respectively, where $J_{80 \mathrm{~K}}$ and $J_{150 \mathrm{~K}}$ are the moments measured at $80 \mathrm{~K}$ and $150 \mathrm{~K}$, respectively. A ratio $\delta_{\mathrm{FC}} / \delta_{\mathrm{ZFC}}>2.0$ passes the Moskowitz test and is considered to be an indicator of the presence of magnetosome chains, although partial oxidation and mixing can cause intact chains to fail the test $[16,20]$. Magnetization retained through low-temperature cycling is expressed as the memory parameter $f_{\mathrm{LTC}}=J_{\mathrm{LTC}} / J_{0}$, where $J_{0}$ and $J_{\mathrm{LTC}}$ are respectively the room-temperature magnetization measured before and after cycling the samples to $10 \mathrm{~K}$.

\subsection{Ferromagnetic resonance spectroscopy}

Ferromagnetic resonance spectra were acquired using an X-band Bruker ESP 300E EPR Spectrometer housed at Caltech. Except for particularly strong samples, microwave power was set at $640 \mu \mathrm{W}$ and spectra were integrated over three sweeps of the applied field from 0 to $600 \mathrm{mT}$. For the strongest samples, microwave power was set at $64 \mu \mathrm{W}$ and one sweep was performed. The spectra were measured at $\sim 9.8 \mathrm{GHz}$. For $77 \mathrm{~K}$ measurements, samples were loaded in a 
quartz glass dewar filled with liquid nitrogen and spectra were measured at $\sim 9.4 \mathrm{GHz}$.

FMR spectra are rich in detail; to extract all the information they convey, they must be examined individually. As a first order summary of spectral characteristics, we use three parameters [20]: $g_{\text {eff }}, A$, and $\Delta B_{\text {FWHM }}$ (Fig. 2). The effective g-factor, $g_{\text {eff, }}$ is the gfactor associated with maximum absorption which is

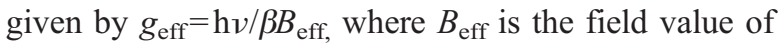
maximum absorption. The asymmetry ratio is defined as $A=\Delta B_{\text {high }} / \Delta B_{\text {low }}$, where $\Delta B_{\text {high }}=B_{\text {high }}-B_{\text {eff }}, \Delta B_{\text {low }}=$ $B_{\text {eff }}-B_{\text {low }}$, and $B_{\text {high }}$ and $B_{\text {low }}$ are the fields of half maximum absorption at low-field and high-field sides of the absorption peak, respectively. The full width at half maximum, $\Delta B_{\mathrm{FWHM}}$, is defined as $\Delta B_{\mathrm{FWHM}}=B_{\mathrm{high}}$ $+B_{\text {low. }}$ Although all these parameters are derived from the integrated absorption spectrum, FMR spectra are generally displayed as derivative spectra, which reveal much greater detail upon inspection. The empirical parameter á, generated by linear discriminant analysis [33] to maximize the difference between magnetosome chains and detrital magnetite in $A$ vs. $\Delta B_{\mathrm{FWHM}}$ space and discussed at length later, is defined as $\alpha=0.17 \mathrm{~A}+9.8 \times 10^{-4} \Delta B_{\mathrm{FWHM}} / \mathrm{mT}$.

\subsection{Simulation of ferromagnetic resonance spectra}

We used MATLAB code, written following Griscom [34,35], to simulate FMR spectra and thereby allow us to understand the relationship between the empirical parameters and physical parameters. We assume that the

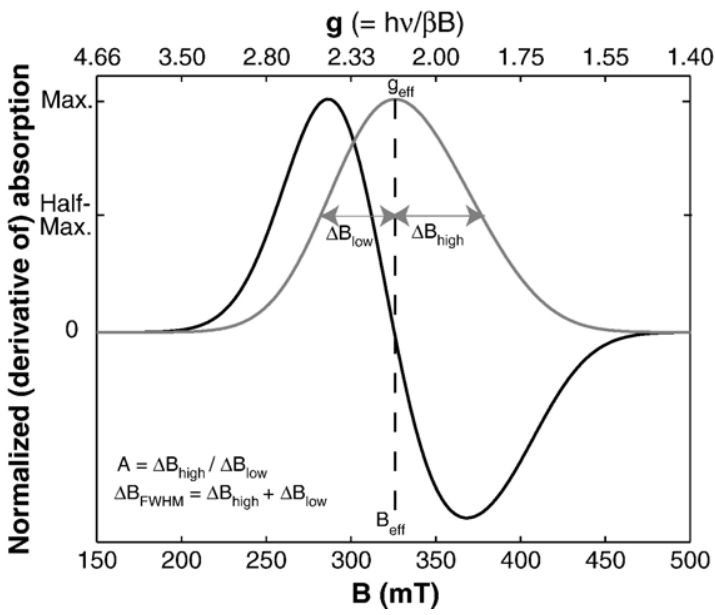

Fig. 2. Definitions of basic FMR parameters. Illustrated on a synthetic FMR spectrum of non-interacting, equidimensional magnetite $\left(g=2.12, \sigma=30 \mathrm{mT}\right.$, cubic $\left.B_{\text {an }}=-55 \mathrm{mT}, K_{2} / K_{1}=0.21\right)$. The dark line shows the derivative spectrum and the light line shows the integrated spectrum. simulated material is a powder of dilute single-domain particles, small with respect to the microwave skin depth ( $\sim 5 \mu \mathrm{m}$ in magnetite [34]), with the particles uniformly distributed in all orientations, and that, at resonance, the orientation of the moment vector approximates the field orientation. Magnetostatic interactions were neglected except as a source of anisotropy, and all anisotropy, whether arising from magnetocrystalline anisotropy, shape anisotropy, or particle arrangement, was treated through a single pair of anisotropy constants, $K_{1}$ and $K_{2}$.

The orientation of an individual particle is defined by the angles $\theta$ and $\varphi$, where $\theta$ is the angle between the applied DC field and the axis of the particle and $\varphi$ is the angle of the axis out of the plane defined by the microwave field and the applied DC field. For a particle with uniaxial anisotropy, the first-order resonance condition (derived from the anisotropy energy $E=K_{1}$ $\sin ^{2} \theta+K_{2} \sin ^{4} \theta$ following the approaches of [18,35]) is given by

$$
\begin{aligned}
B_{\text {res,uni }}(\theta)= & B_{\text {true }}-\frac{1}{2} B_{\text {an }} \times\left[3 \cos ^{2} \theta-1\right. \\
& \left.+\left(K_{2} / K_{1}\right)\left(8 \cos ^{2} \theta \sin ^{2} \theta-2 \sin ^{4} \theta\right)\right]
\end{aligned}
$$

where $B_{\text {true }}=\mathrm{h} \nu / \beta g_{\text {true }}$ is the resonance field in the absence of anisotropy, $B_{\text {an }}$ is the anisotropy field $2 K_{1} /$ $M_{\mathrm{s}}, K_{1}$ and $K_{2}$ are the first-order and second-order uniaxial anisotropy constants, and $M_{\mathrm{s}}$ is the saturation magnetization. For cubic anisotropy, the resonance condition of [35] was used.

To compute the powder absorption at applied field $B_{\text {app }}$, a Gaussian broadening function of linewidth $\sigma$ is applied and spectra are numerically integrated over all solid angles:

$$
\begin{aligned}
A\left(B_{\mathrm{app}}\right)= & \int_{\theta=0}^{\pi / 2} \int_{\phi=0}^{2 \pi} \frac{\exp \left(-\left(B_{\mathrm{app}}-B_{\mathrm{res}}(\theta, \phi)\right)^{2} / 2 \sigma^{2}\right)}{\sqrt{2 \pi \sigma}} d \phi \\
& \times \sin \theta d \theta
\end{aligned}
$$

Several physical effects are subsumed in the Gaussian broadening; in natural samples, a major cause of broadening is heterogeneity in particle size, shape, and arrangement [36].

While not capable of fitting our observed spectra perfectly, nonlinear least square fitting using these model spectra provides reasonably good approximations. For almost all our samples, second-order uniaxial fits were better than second-order cubic fits, and they never were significantly worse. We would expect this to be the case for samples in which magnetostatic interactions or shape anisotropy dominated the internal magnetic environment. 


\subsection{Transmission electron microscopy (TEM)}

We prepared replicas, composed of a 1-2 nm thick metal thin film of $95 \%$ platinum and $5 \%$ carbon and a supporting carbon film 15-20 nm thick, of the synthetic magnetite dilutions to allow us to remove the sucrose dilutant while preserving the relationship between the dilutant and the magnetite. Ten microliters of $\sim 20 \mathrm{mg}$ of powder dispersed in $99 \%$ pure methanol was transferred onto a freshly cleaved mica thin plate. As the powder settled, the fluid was removed by blotting with filter paper. The replica was prepared in a freeze-fracture unit (Baltec 60, Lichtenstein) at room temperature under high vacuum (10-6 Torr), as described in [37], by depositing a fine granular Pt-C film from an evaporating source at an incident angle of $30^{\circ}$ and a distance of $15 \mathrm{~cm}$ to the mica plate. Variable densities of Pt-C accumulate along the topographic slopes of the specimen that are facing, oblique to, or shielded from the source, emphasizing the topographic contrast for TEM imaging. To strengthen the ultra-thin Pt films and preserve their integrity during cleaning steps, a supporting carbon film was immediately condensed on the Pt film by a vertical electron beam gun sputtering a carbon rod perpendicular to and $12 \mathrm{~cm}$ away from the surface of the specimen. The replicas were cleaned with distilled water to remove the sucrose and transferred onto 200 mesh formvar-coated $\mathrm{Cu}$ grids.

Imaging was carried out in bright-field illumination with a JEOL JEM-2000FX transmission electron microscope, at an accelerating voltage of $80 \mathrm{kV}$ and magnifications from 5000×-50,000×, and a Gatan 792 Bioscan $1 \mathrm{k} \times 1 \mathrm{k}$ Wide Angle Multiscan CCD camera.

\section{Results}

\subsection{Lysis of magnetotactic bacteria}

When comparing different arrangements of similar particles, ARM susceptibility quantifies magnetostatic interactions. Three-dimensional magnetostatic interactions shield particles from the DC biasing field, which causes materials with greater interactions to exhibit lower ARM susceptibility. (This is not the case for materials with one-dimensional magnetostatic interactions, as in magnetosome chains.) As expected from these considerations, the ARM susceptibilities of the samples are ordered: intact AMB-1 (samples A1a and A1b) $>$ ultrasonically disrupted AMB-1 (samples A2a and samples A2b) $>$ dispersed, SDS-treated AMB-1 (sample A3c) $>$ concentrated, SDS-treated AMB-1 (samples A3b and A3a) (Fig. 3A, Table 1).
Ferromagnetic resonance spectra of the disrupted AMB-1 samples reflect a shift from linear chains to highly interacting clumps (Fig. 3B, Table 1). The ultrasonically disrupted AMB-1 (sample A2a) exhibits little change in spectrum shape or in the spectral parameters compared to intact AMB-1 (sample A1a). This suggests that, although the magnetostatic interactions as revealed by the ARM curves have increased relative to the intact cells, the local magnetic environment felt by each individual crystal remains dominated by head-to-tail axial interactions; the chains are still intact, although they are no longer separated from one another by cell material (see Fig. 1 of [25]). In contrast, the spectrum of the SDS-treated cells exhibits a high-field extended asymmetry and high $g_{\text {eff }}$ (sample A3a), consistent with the observations of Weiss et al. [20].

The diluted SDS-treated cells (sample A3c) exhibit a spectrum that more closely resembles that of the intact and ultrasonicated samples, although the spectrum of $\mathrm{A} 3 \mathrm{c}$ is a bit wider. One key trait of this spectrum that is not readily apparent from the parameterization is its degree of sharpness. The derivative spectra of intact and ultrasonicated AMB-1 (A1a and A2a) have two local maxima, which indicate a low degree of peak broadening caused by heterogeneous particle arrangements. Each particle experiences roughly the same magnetic anisotropy produced by particle interactions within the chain, because the magnetite chains are produced under biological control. In contrast, the dispersed, SDStreated AMB-1 (A3c) has only one local maximum, likely because the particles are arranged in onedimensional strings that form through physical processes and therefore have more heterogeneous magnetic anisotropy. The same effect is seen in comparing Fig. 1C and $\mathrm{E}$.

All the AMB-1 spectra are fairly well fit by secondorder uniaxial model spectra. The intact, ultrasonicated, and dispersed SDS-treated samples have $B_{\text {an }}$ values around $80 \mathrm{mT}$. Following the method of Butler and Banerjee [38,39], this value is equivalent to the shape anisotropy of an elongate magnetite crystal with a 2.2:1 length-to-width ratio. Since the crystals produced by AMB-1 are equidimensional, the apparent shape anisotropy must be due to linear magnetostatic interactions. The $-81 \mathrm{mT} B_{\text {an }}$ value of the concentrated SDStreated sample is equivalent to a single crystal with a 1:1.1 length-to-width ratio, and may be due to planar magnetostatic interactions, as on the surface of a clump. All SDS-treated samples have larger $\sigma$ values than intact and ultrasonicated samples, which likely reflect greater heterogeneity of particle arrangement. 

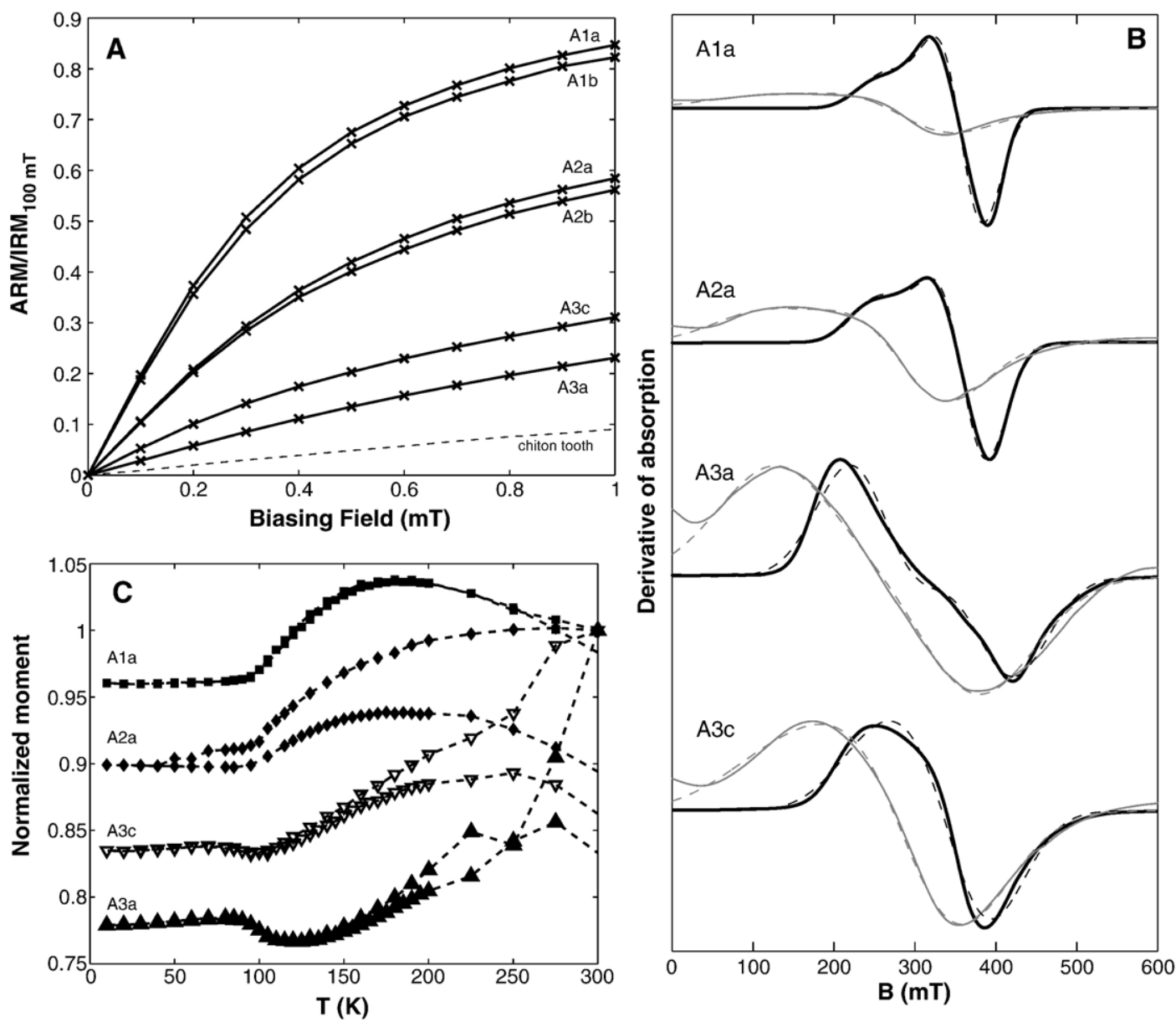

Fig. 3. Measurements of intact and altered AMB-1. (A) ARM acquisition curves, (B) ferromagnetic resonance spectra, and (C) low-temperature cycling curves of cultures of AMB-1. In (A), the lower dashed line is a chiton tooth standard for highly interacting magnetite. In (B), thick lines represent room-temperature measurements, thin lines represent $77 \mathrm{~K}$ measurements (where performed), and dashed lines indicate spectral fits.

The FMR spectra measured at $77 \mathrm{~K}$ are consistent with these findings (Fig. 3B, Table 2). Below the Verwey transition, which, as measured in the $\mathrm{FC}$ and ZFC curves (not shown; see also [20]), occurs at $\sim 100 \mathrm{~K}$ in magnetite from AMB-1, magnetite shifts from a cubic crystal system to a monoclinic crystal system and experiences a large increase in magnetocrystalline anisotropy, with the anisotropy constants becoming positive $[40,41]$. Thus, the spectra broaden considerably, $g_{\text {eff }}$ shifts to higher values, and $A$ becomes

Table 1

Summary of FMR and magnetic parameters of bacterial samples

\begin{tabular}{|c|c|c|c|c|c|c|c|c|c|c|c|c|c|}
\hline \multicolumn{2}{|c|}{ Sample } & \multicolumn{4}{|c|}{ FMR spectrum } & \multicolumn{4}{|c|}{ FMR uniaxial fit } & \multicolumn{4}{|c|}{ Magnetic properties } \\
\hline ID & Treatment & $g_{\text {eff }}$ & $A$ & $\begin{array}{l}\Delta B \\
(\mathrm{mT})\end{array}$ & $\alpha$ & $\mathrm{g}_{\text {fit }}$ & $\begin{array}{l}B_{\mathrm{an}} \\
(\mathrm{mT})\end{array}$ & $K_{2} / K_{1}$ & $\begin{array}{l}\sigma \\
(\mathrm{mT})\end{array}$ & $\begin{array}{l}k_{\mathrm{ARM}} / \mathrm{IRM} \\
(\mathrm{mm} / \mathrm{A})\end{array}$ & $\delta_{\mathrm{ZFC}}$ & $\delta_{\mathrm{FC}} / \delta_{\mathrm{ZFC}}$ & $\mathrm{f}_{\mathrm{LTC}}$ \\
\hline Ala & Intact & 2.01 & 0.79 & 96 & 0.23 & 2.08 & 81 & -0.13 & 27 & 2.48 & 0.14 & 2.44 & 0.98 \\
\hline $\mathrm{A} 1 \mathrm{~b}$ & Intact, pwd. & 2.01 & 0.83 & 93 & 0.23 & 2.08 & 86 & -0.20 & 29 & 2.36 & & & \\
\hline $\mathrm{A} 2 \mathrm{a}$ & Ultra. & 2.01 & 0.73 & 112 & 0.23 & 2.10 & 84 & -0.06 & 30 & 1.34 & 0.16 & 1.94 & 0.89 \\
\hline $\mathrm{A} 2 \mathrm{~b}$ & Ultra., pwd. & 2.02 & 0.78 & 103 & 0.23 & 2.09 & 85 & -0.14 & 31 & 1.31 & & & \\
\hline $\mathrm{A} 3 \mathrm{a}$ & SDS, conc. & 2.25 & 1.13 & 208 & 0.40 & 2.20 & -81 & 0.43 & 48 & 0.36 & 0.37 & 1.21 & 0.83 \\
\hline $\mathrm{A} 3 \mathrm{~b}$ & SDS, pwd $1 \mathrm{~m}$ & 2.14 & 0.89 & 188 & 0.34 & 2.17 & -60 & 0.65 & 48 & 0.36 & & & \\
\hline $\mathrm{A} 3 \mathrm{c}$ & SDS, pwd $5 \mathrm{~m}$ & 2.07 & 0.76 & 148 & 0.27 & 2.14 & 79 & 0.01 & 50 & 0.66 & 0.26 & 1.39 & 0.86 \\
\hline
\end{tabular}


Table 2

Summary of $77 \mathrm{~K}$ FMR parameters of bacterial and synthetic magnetite samples

\begin{tabular}{|c|c|c|c|c|c|c|c|c|}
\hline \multirow[t]{2}{*}{ ID } & \multicolumn{4}{|c|}{ FMR spectrum } & \multicolumn{4}{|c|}{ FMR uniaxial fit } \\
\hline & $g_{\text {eff }}$ & A & $\Delta B(\mathrm{mT})$ & $\alpha$ & $\mathrm{g}_{\text {fit }}$ & $\mathrm{B}_{\mathrm{an}}(\mathrm{mT})$ & $K_{2} / K_{1}$ & $\sigma(\mathrm{mT})$ \\
\hline A1a & 2.47 & 0.64 & 215 & 0.32 & 2.76 & 149 & -0.04 & 64 \\
\hline Alb & 2.46 & 0.64 & 201 & 0.31 & 2.75 & 144 & -0.04 & 57 \\
\hline $\mathrm{A} 2 \mathrm{a}$ & 2.53 & 0.69 & 211 & 0.32 & 2.79 & 134 & 0.02 & 59 \\
\hline $\mathrm{A} 2 \mathrm{~b}$ & 2.48 & 0.68 & 206 & 0.32 & 2.73 & 138 & -0.02 & 59 \\
\hline A3a & 2.59 & 0.97 & 266 & 0.43 & 2.63 & 97 & 0.46 & 75 \\
\hline $\mathrm{A} 3 \mathrm{c}$ & 2.45 & 0.83 & 214 & 0.35 & 2.59 & 134 & -0.08 & 71 \\
\hline $\mathrm{T} 1 \mathrm{c}$ & 2.16 & 0.77 & 297 & 0.42 & 2.33 & 144 & 0.22 & 71 \\
\hline $\mathrm{T} 2 \mathrm{~b}$ & 2.26 & 0.80 & 299 & 0.43 & 2.42 & 139 & 0.25 & 72 \\
\hline T3c & 2.20 & 0.71 & 228 & 0.34 & 2.40 & 147 & 0.01 & 63 \\
\hline
\end{tabular}

lower, reflecting the positive anisotropy. The last effect is strongest in sample A3a, which shifts from high field asymmetry to mild low field asymmetry, presumably as the anisotropy field comes to dominate magnetostatic interactions. A3a continues, however, to have higher $g_{\text {eff }}, A$, and $\Delta B_{\text {FWHM }}$ than the other samples.

Low-temperature data (Fig. 3C; Table 1) exhibit increasing loss of remanence upon warming or cooling through the Verwey transition with increasing magnetostatic interactions (decreasing ARM susceptibility), consistent with [42]. In addition, the $\delta_{\mathrm{FC}} / \delta_{\mathrm{ZFC}}$ ratio decreases with chain breakdown and only the intact AMB- 1 cells pass a Moskowitz test with $\delta_{\mathrm{FC}} / \delta_{\mathrm{ZFC}}>2$. Increased chain breakdown also increases the fraction of remanence loss on cooling and re-warming through the Verwey transition. Curiously, the SDS-treated cells, both concentrated and dispersed, experience a slight increase in remanence when they are cooled through the Verwey transition, while the samples with intact chains lose remanence across the Verwey transition.

\subsection{Dilutions of abiogenic magnetite}

To test our hypothesis that the low-field extended asymmetry of the dispersed, lysed AMB-1 is a product of physical string formation, we performed analogous experiments using synthetic magnetite. As expected, dilution of the powder decreases three-dimensional magnetostatic interactions, which is reflected in increased ARM susceptibility (Fig. 4A, Table 3). This pattern is consistent when comparing different ratios of magnetite to sucrose and when comparing shaken mixtures to mixtures ground with a mortar and pestle for four minutes. The ARM susceptibility of the samples is ordered: $400 \mathrm{ppm}$ ground mixture (sample T3c) $>0.6 \%$ ground mixture (sample T2c) $>400 \mathrm{ppm}$ shaken mixture (sample $\mathrm{T} 3 \mathrm{a}$ ) $>6 \%$ ground mixture (sample
T1c) $>0.6 \%$ shaken mixture (sample T2a) $>6 \%$ shaken mixture (sample T1a), although the ARM/IRM ratios of $\mathrm{T} 1 \mathrm{c}$ cross above those of T3a and T2c at higher biasing fields.
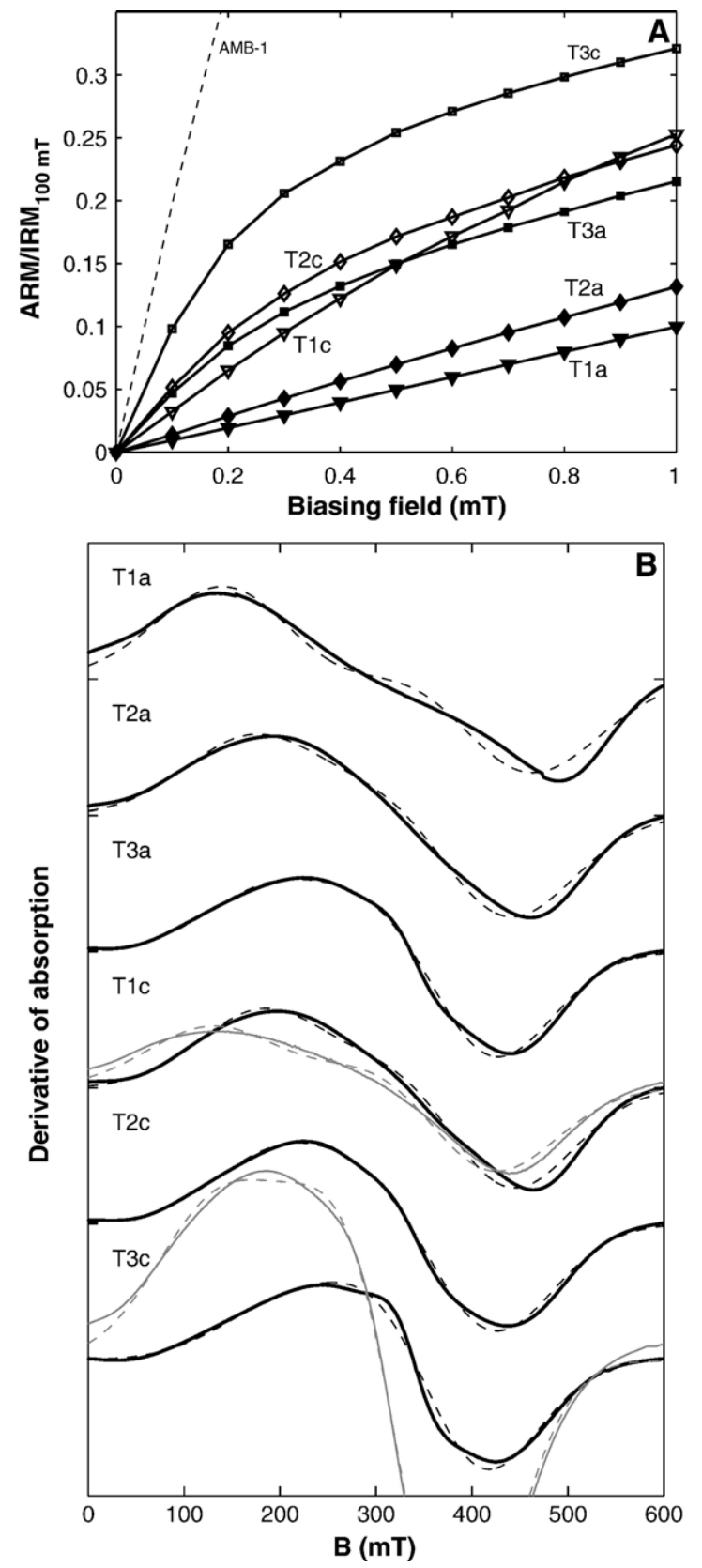

Fig. 4. Measurements of synthetic magnetite. (A) ARM acquisition curves and (B) ferromagnetic resonance spectra of dilutions of synthetic magnetite powder TMB-100. In (A), the upper dashed line is intact AMB-1. The chiton tooth standard shown in Fig. 2a closely follows the line for sample T1a. In (B), thick lines represent roomtemperature measurements, thin lines represent $77 \mathrm{~K}$ measurements, and dashed lines indicate spectral fits. 
Table 3

Summary of FMR and magnetic parameters of synthetic magnetite samples

\begin{tabular}{|c|c|c|c|c|c|c|c|c|c|c|}
\hline \multicolumn{2}{|c|}{ Sample } & \multicolumn{4}{|c|}{ FMR spectrum } & \multicolumn{4}{|c|}{ FMR uniaxial fit } & \multirow{2}{*}{$\begin{array}{l}k_{\mathrm{ARM}} / \mathrm{IRM} \\
(\mathrm{mm} / \mathrm{A})\end{array}$} \\
\hline ID & $\begin{array}{l}\text { Conc. (ppm)/ } \\
\text { Pwd time (min) }\end{array}$ & $g_{\text {eff }}$ & $A$ & $\begin{array}{l}\Delta B \\
(\mathrm{mT})\end{array}$ & $\alpha$ & $\mathrm{g}_{\text {fit }}$ & $\begin{array}{l}B_{\text {an }} \\
(\mathrm{mT})\end{array}$ & $K_{2} / K_{1}$ & $\begin{array}{l}\sigma \\
(\mathrm{mT})\end{array}$ & \\
\hline T1a & $60,000 / 0$ & 2.37 & 1.12 & 339 & 0.52262 & 2.31 & 114 & 0.59 & 78 & 0.11 \\
\hline $\mathrm{T} 1 \mathrm{~b}$ & $60,000 / 1$ & 2.21 & 0.96 & 282 & 0.43956 & 2.26 & 117 & 0.34 & 74 & 0.15 \\
\hline $\mathrm{T} 1 \mathrm{c}$ & $60,000 / 4$ & 2.11 & 0.87 & 267 & 0.40956 & 2.19 & 123 & 0.25 & 69 & 0.40 \\
\hline $\mathrm{T} 2 \mathrm{a}$ & $6000 / 0$ & 2.17 & 0.91 & 274 & 0.42322 & 2.24 & 126 & 0.24 & 73 & 0.18 \\
\hline $\mathrm{T} 2 \mathrm{~b}$ & $6000 / 1$ & 2.16 & 0.92 & 259 & 0.41022 & 2.23 & 123 & 0.20 & 73 & 0.24 \\
\hline $\mathrm{T} 2 \mathrm{c}$ & $6000 / 4$ & 2.10 & 0.85 & 228 & 0.36794 & 2.19 & 121 & 0.09 & 69 & 0.64 \\
\hline T3a & $400 / 0$ & 2.09 & 0.83 & 230 & 0.36650 & 2.19 & 131 & 0.06 & 67 & 0.59 \\
\hline $\mathrm{T} 3 \mathrm{~b}$ & $400 / 1$ & 2.09 & 0.83 & 222 & 0.35866 & 2.19 & 132 & 0.03 & 67 & 0.74 \\
\hline $\mathrm{T} 3 \mathrm{c}$ & $400 / 4$ & 2.07 & 0.83 & 201 & 0.33808 & 2.17 & 131 & -0.06 & 64 & 1.23 \\
\hline
\end{tabular}

The FMR spectra of these samples reveal the same trend, with $g_{\text {eff }}$ and $\Delta B_{\mathrm{FWHM}}$ decreasing in the same order that ARM susceptibilities decrease (Figs. 4B, 5 and Table 3). The $6 \%$ shaken mixture is markedly different from the other mixtures. It alone exhibits highfield extended asymmetry, rather than low-field extended asymmetry. The other mixtures exhibit low-field extended asymmetry, which we suggest is due to the formation of strings of particles. If the shift to low-field extended asymmetry were due to changes in crystal shape upon powdering, it would not be present in samples diluted only by shaking. The fitted $B_{\text {an }}$ of the particles is equivalent to that of single magnetite crystals with length-to-width ratios of $\sim 3: 1$, even though TEM images show largely equidimensional crystals. TEM images of sample T2c (Fig. 6) indicate that, as predicted, many of the crystals are arranged in strings. While the

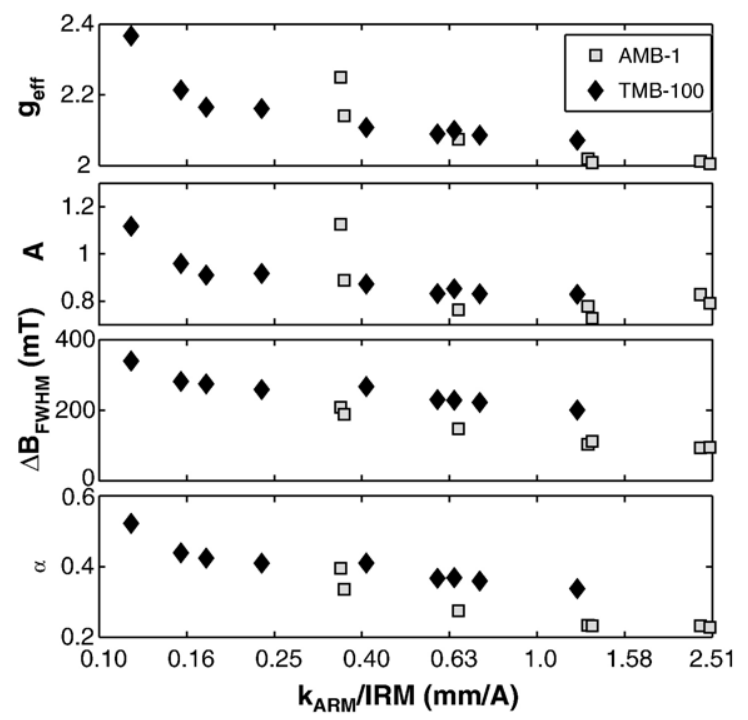

Fig. 5. Semi-log plots of the FMR parameters of the synthetic magnetite and AMB-1 samples against $k_{\mathrm{ARM}} / \mathrm{IRM}$. strings are much more irregular than biogenic chains, they produce the appropriate magnetic anisotropy to generate FMR spectra with low-field extended asymmetry. Their heterogeneity, however, leads to a broader spectrum than those produced by bacterial magnetite chains; the strings of magnetite have only a single lowfield derivative peak, in contrast to the double peak of the bacterial chains.

\subsection{Sedimentary samples}

The sedimentary samples studied come from three sources: the Bahamas, the southern Atlantic Ocean, and Mono Basin, CA. The range of FMR parameters from these samples are shown in Fig. 7 and in Table 4. Although no samples from Andros Island have been previously studied using FMR, magnetic measurements and electron microscopy on a small number of samples from elsewhere in the Great Bahama Bank suggest that magnetotactic bacteria are common at the sediment/ water interface [43], and data from Pliocene-Pleistocene sediments on San Salvador Island and from the Clino core drilled through the Great Bahama Bank indicate that biogenic magnetite can be preserved in sediments that have undergone diagenesis $[20,44,45]$.

The spectra of the Bahamian samples all exhibit multiple derivative maxima and low-field extended asymmetry, with $A$ between 0.71 and $0.86, g_{\text {eff }}$ between 2.01 and 2.11, and $\Delta B_{\text {FWHM }}$ between 109 and $192 \mathrm{mT}$ (Fig. 8A). Based on the criteria of Weiss et al. [20], these traits are all consistent with the presence of intact magnetosome chains (Fig. 3). Although many of these parameters also characterize the strings of crystals from lysed cells described above (Fig. 4), the secondary absorption peaks of these Bahamian samples resemble that observed for intact magnetosome chains. In addition, all samples have strong paramagnetic resonances from $\mathrm{Fe}^{+3}(g=4.3)$ and 

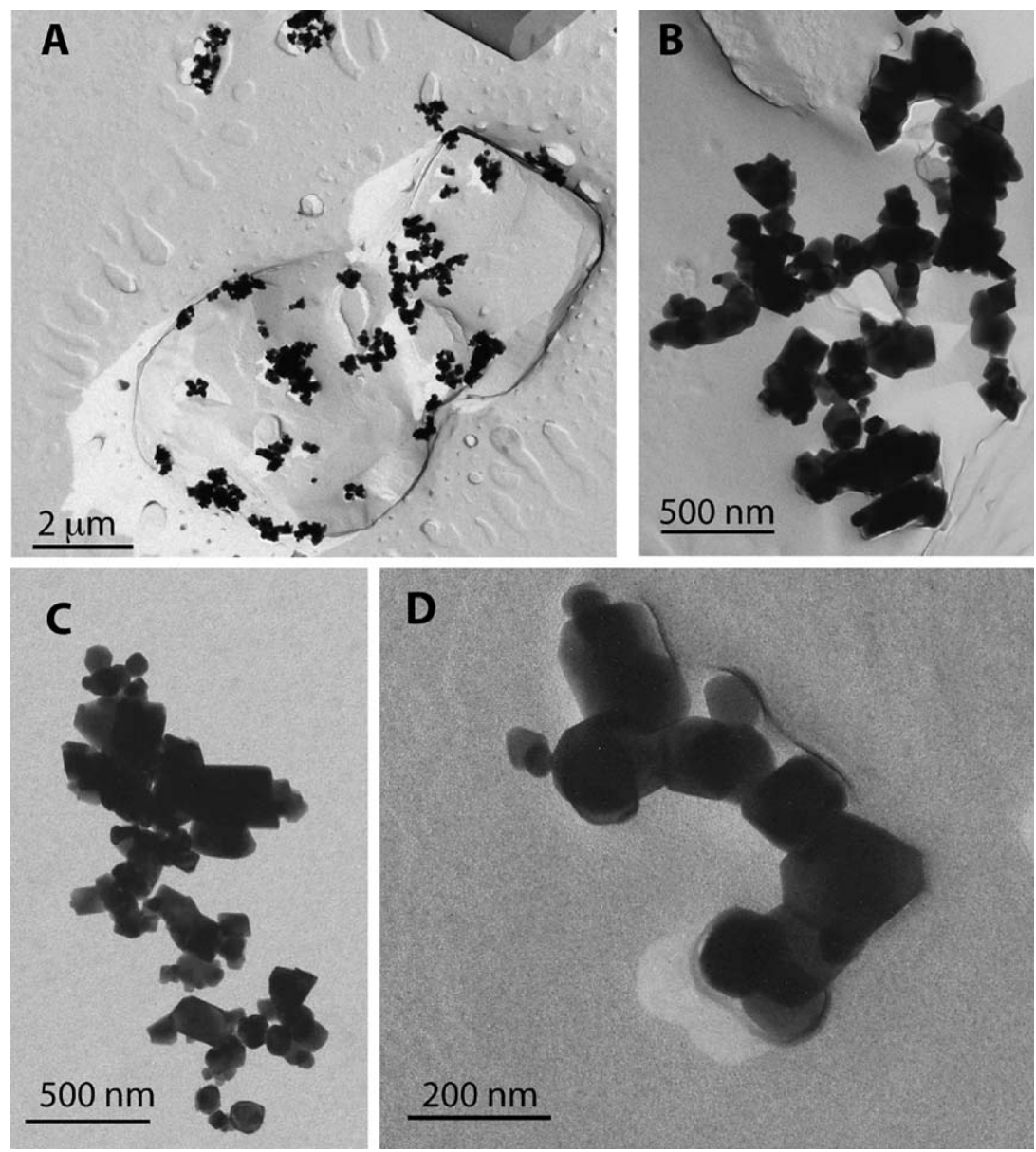

Fig. 6. Transmission electron micrographs of Pt-C replica of synthetic magnetite powder TMB-100 diluted at 6000 ppm in sucrose. The bright material is the Pt-C replica of the sucrose dilutant, while the dark crystals are magnetite. (A) shows the association between clumps and strings of magnetite particles and the surface of sucrose crystals. (B), (C), and (D) show higher resolution images of the clumps and strings.
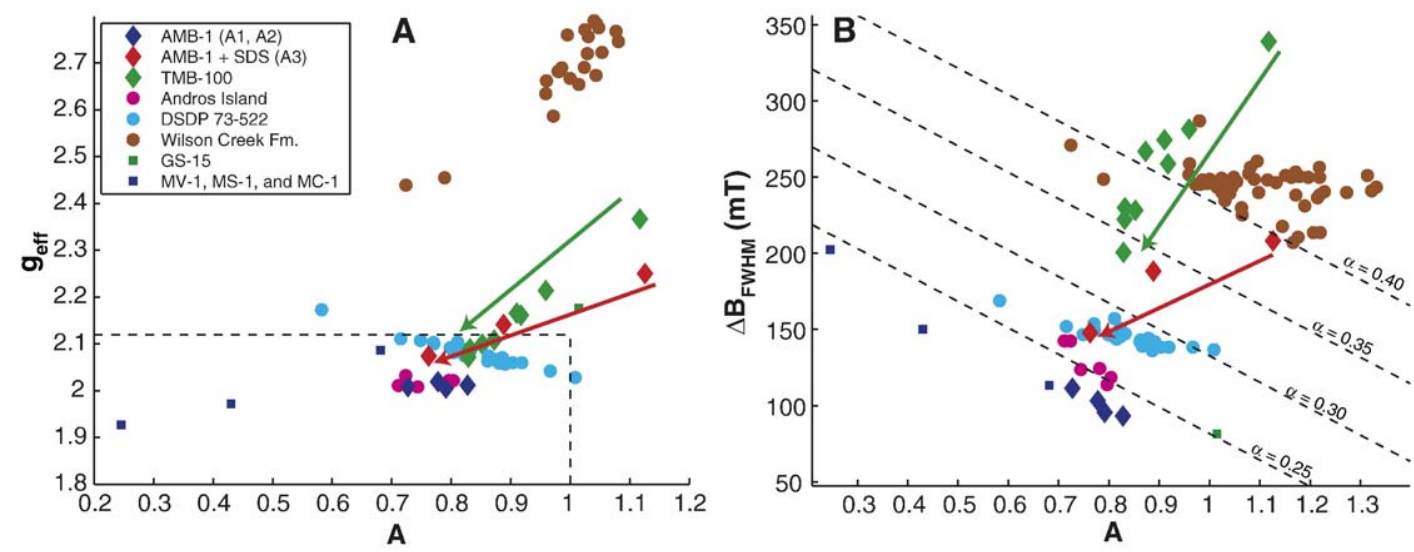

Fig. 7. Summary of FMR parameters. Plots of (A) effective g-factor vs. asymmetry ratio $A$, in the manner of Weiss et al. [20], and (B) full width at half maximum $\left(\Delta B_{\mathrm{FWHM}}\right)$ vs. $A$. In (A), the dashed lines demarcate the area defined by Weiss et al. [20] as being the domain of magnetosome chains, with $A<1$ and $g_{\text {eff }}<2.12$. In (B), the dashed lines are contours of constant $\alpha$ values, where $\alpha$ is defined in the text. Red and green arrows highlight the dilution trends for, respectively, SDS-treated AMB-1 and TMB-100. Parameters for Geobacter metallireducens GS-15, which precipitates extracellular superparamagnetic magnetite, and for the magnetotactic bacteria strains MV-1, MS-1, and MC-1 (arranged in order of increasing $A$ ) are derived from the data of $[20]$. 
Table 4

Summary of parameters of environmental samples

\begin{tabular}{|c|c|c|c|c|c|}
\hline \multirow[t]{2}{*}{ Sample } & \multicolumn{4}{|c|}{ FMR spectrum } & \multirow{2}{*}{$\begin{array}{l}\mathrm{k}_{\mathrm{ARM}} / \mathrm{IRM} \\
(\mathrm{mm} / \mathrm{A})\end{array}$} \\
\hline & $g_{\text {eff }}$ & $A$ & $\begin{array}{l}\Delta B \\
(\mathrm{mT})\end{array}$ & $\alpha$ & \\
\hline \multicolumn{6}{|c|}{ Andros island C51 } \\
\hline $6 \mathrm{~cm}$ & 2.03 & 0.72 & 143 & 0.26 & 2.54 \\
\hline $14 \mathrm{~cm}$ & 2.01 & 0.71 & 143 & 0.26 & 2.55 \\
\hline $22 \mathrm{~cm}$ & 2.01 & 0.78 & 124 & 0.25 & 3.08 \\
\hline $31 \mathrm{~cm}$ & 2.01 & 0.74 & 124 & 0.25 & 2.49 \\
\hline $42 \mathrm{~cm}$ & 2.02 & 0.80 & 118 & 0.25 & 1.21 \\
\hline $52 \mathrm{~cm}$ & 2.02 & 0.80 & 114 & 0.25 & 0.96 \\
\hline \multicolumn{6}{|c|}{ Andros island } \\
\hline Mean & 2.04 & 0.77 & 140 & 0.27 & 1.97 \\
\hline Min. & 2.01 & 0.71 & 109 & 0.25 & 0.96 \\
\hline Max. & 2.11 & 0.86 & 192 & 0.32 & 3.08 \\
\hline \multicolumn{6}{|c|}{$D S D P 73-522$} \\
\hline Mean & 2.08 & 0.83 & 146 & 0.28 & 1.31 \\
\hline Min. & 2.03 & 0.58 & 136 & 0.26 & 1.07 \\
\hline Max. & 2.17 & 1.01 & 169 & 0.31 & 1.62 \\
\hline \multicolumn{6}{|c|}{ Wilson Creek } \\
\hline Mean & 2.88 & 1.11 & 242 & 0.43 & 0.25 \\
\hline Min. & 2.58 & 0.96 & 207 & 0.40 & 0.25 \\
\hline Max. & 3.34 & 1.33 & 287 & 0.47 & 0.38 \\
\hline
\end{tabular}

$\mathrm{Mn}^{+2}(g=2.0)$. The latter obscure the magnetite signal in the derivative curve, but due to the sharp peaks of the $\mathrm{Mn}^{+2}$ sextet, the $\mathrm{Mn}^{+2}$ signal is readily removed by curve smoothing (Fig. 7A).

A close examination of core C51 reveals changes with depth suggestive of the progressive alteration of magnetosome chains. The first local maximum of the derivative spectrum in the $6 \mathrm{~cm}$ and $14 \mathrm{~cm}$ samples is well defined. In the 22 and $31 \mathrm{~cm}$ samples, it is present but diminished in strength relative to the second local maximum. In the 42 and $52 \mathrm{~cm}$ samples, the first local maximum is not present, and ARM susceptibility is markedly diminished compared to shallower samples (Fig. 8B and C). These changes suggest the progressive degradation of magnetosome chains with depth, although they could alternatively represent the mixing of two discrete magnetic materials in the upper sediments, and the loss of one of these with depth.

The deep-sea sediments studied contain magnetofossils of a variety of morphologies, some partially dissolved [29]. TEM images of magnetic extracts from these samples often produced interlocking meshes and linked strings more closely resembling those present in our dilutions of abiogenic magnetite than the chains present in intact bacteria. Excluding one outlier, all the DSDP Leg 73 samples have $A$ of $0.72-1.01, g_{\text {eff }}$ of
2.03-2.11, and $\Delta B_{\mathrm{FWHM}}$ of $136-157 \mathrm{mT}$. A typical spectrum is shown in Fig. 9A. It is similar to that of the dispersed, SDS-treated AMB-1 (sample A3c; dashed line in Fig. 9A). Like sample A3c, it lacks the second local maximum characteristic of intact bacterial cultures and the laminated Bahamian sediments. Instead, it is consistent with more heterogeneous sediments where the magnetization is dominated by bacterial magnetite in which many of the chains have been broken up but the particles have largely remained associated in roughly linear low-energy configurations - precisely what TEM images [29] reveal.

The samples from the Pleistocene Wilson Creek Formation (Fig. 9B) were deposited in Lake Russell, the late Pleistocene predecessor of modern Mono Lake. Microbiological research in Mono Lake has revealed a variety of magnetotactic organisms (C. Z. Nash, unpublished results), but due to the basin's location in the Sierra Nevada, sedimentary flux into the basin and thus sediment magnetization is dominated by detrital material derived from Sierran intrusive bodies. These samples exhibit a broad range of $A$ values (0.73-1.33; all but two compositionally anomalous samples, an ash and sand lens, have $A \geq 0.96$ ), have $g_{\text {eff }}$ well above those of all other samples considered in this paper (2.44-3.34; all but the two anomalous samples have $g_{\text {eff }} \geq 2.58$ ), and $\Delta B_{\mathrm{FWHM}}$ larger than the samples dominated by bacterial magnetite (207-287 mT).

\section{Discussion}

Weiss et al. [20] suggested that the magnetosome chain structure led cultures of magnetotactic bacteria to produce FMR spectra with multiple derivative maxima, $A<1$ and $g_{\text {eff }}<2.12$ (the true g-factor for magnetite). Our results support these claims: our bacterial samples with intact chains had multiple maxima, $A \leq 0.84$ and $g_{\text {eff }}=2.02$. With two exceptions, our sedimentary samples in which magnetofossils are believed to be a major carrier of magnetization had $A \leq 0.97$ and $g_{\text {eff }} \leq 2.11$ (Fig. 6A; Tables 1 and 4), although not all had multiple maxima. Yet while $A \leq 1$ and $g_{\text {eff }} \leq 2.12$ may be necessary conditions for a FMR spectrum to be a spectrum of magnetofossils, they are not sufficient. We were able to produce abiogenic samples with $A \leq 0.87$ and $g_{\text {eff }} \leq 2.11$ by dispersing magnetite in a nonmagnetic matrix, thus causing the crystals to string out in minimum energy head-to-tail configurations (Table $3)$. This finding strengthens the claim that $A \leq 1$ and $g_{\text {eff }} \leq 2.12$ reflect linear arrangements of crystals, but indicates that these criteria alone cannot uniquely identify magnetofossils. 

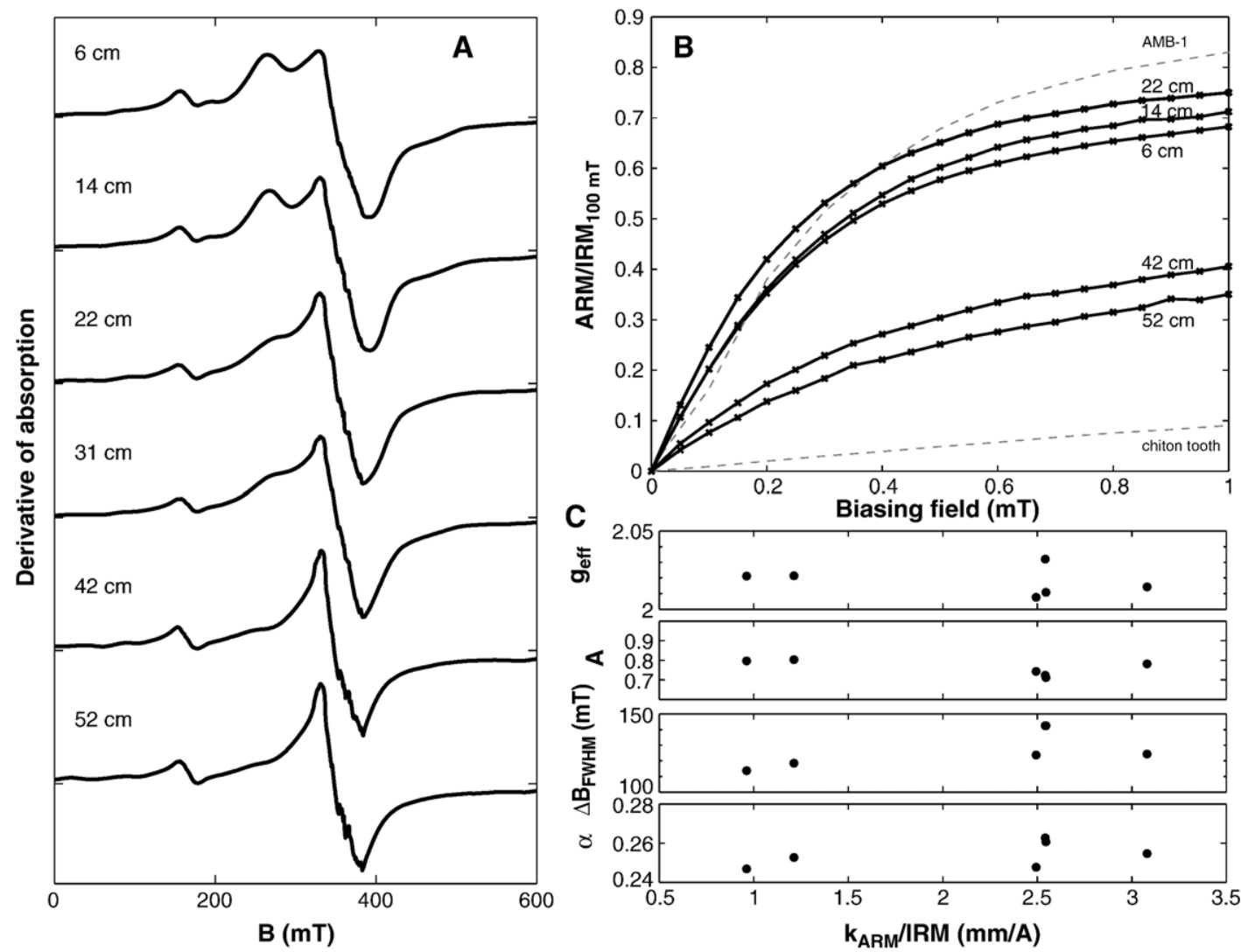

Fig. 8. Measurements through Andros Island core C51. (A) Smoothed ferromagnetic resonance spectra, (B) ARM acquisition curves, and (C) plots of FMR parameters against $k_{\mathrm{ARM}}$ /IRM. In (A), the small peak at $\sim 160 \mathrm{mT}$ is the paramagnetic resonance of $\mathrm{Fe}^{+3}$ and the jaggedness at $\sim 330 \mathrm{mT}$ is the remanent of the $\mathrm{Mn}^{+2}$ resonance, most of which was removed by smoothing. On (B), the upper dashed line is the ARM acquisition curve of intact AMB-1, while the lower dashed line is that of the highly interacting chiton tooth standard. The $31 \mathrm{~cm}$ ARM curve, not shown, nearly follows the $14 \mathrm{~cm}$ curve.

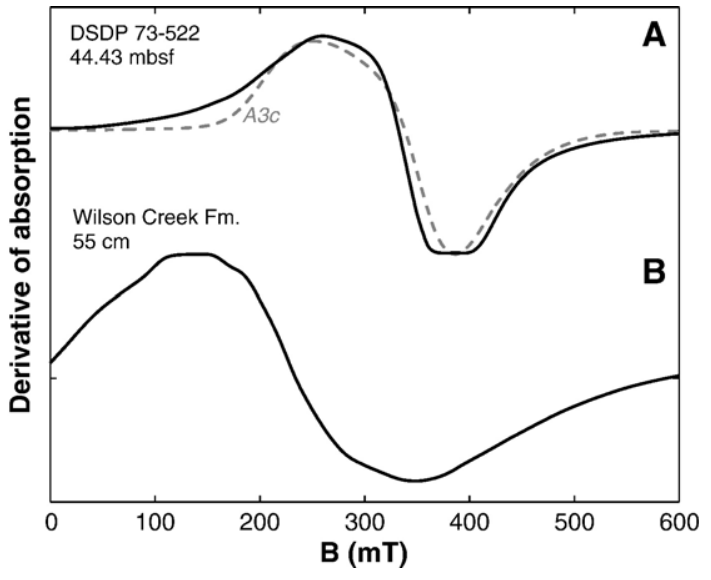

Fig. 9. Representative FMR spectra of (A) Oligocene-Miocene deepsea muds from DSDP Leg 73 Site 522, in which magnetofossils are a major remanence carrier and (B) silts from the Pleistocene Wilson Creek Formation of Mono Basin, which are magnetically dominated by detrital magnetite. In (A), the dashed line shows by way of comparison AMB-1 sample A3c, which was treated with SDS and diluted in sucrose by powdering for $5 \mathrm{~min}$.
Closer examination of the FMR spectra suggests alternative criteria that may better distinguish between biogenic and abiogenic magnetite. Increased $\Delta B_{\mathrm{FWHM}}$ in a spectrum can have multiple causes, including increased anisotropy (increased $\left|B_{\text {an }}\right|$ in model spectra) and greater sample heterogeneity (increased ó in model spectra). Variability among magnetotactic bacteria is largely accounted for by the former: the magnetotactic bacteria strain MV-1, which produces elongate truncated hexaoctahedral crystals, has higher magnetic anisotropy than MS-1 or AMB-1, which produce chains of cubooctahedral crystals [5]. The higher anisotropy both increases $\Delta B_{\mathrm{FWHM}}$ and decreases $A$. Similarly, MS-1 has lower $A$ and higher $\Delta B_{\mathrm{FWHM}}$ than AMB-1, possibly due to better formed crystals and fewer gaps in their chains than AMB-1. In contrast, in abiogenic samples, heterogeneity plays a major role in controlling $\Delta B_{\mathrm{FWHM}}$, and $\Delta B_{\text {FWHM }}$ can vary significantly without corresponding decreases in $A$.

The parameter $\alpha$, defined in Section 3.2, is useful in distinguishing between biogenic and abiogenic samples. 
All the bacterial samples with intact chains, including magnetotactic strains MV-1, MS-1 and MC-1 measured by Weiss et al. [20], possess $\alpha<0.25$, while almost all the lithogenic samples from the Wilson Creek Formation have $\alpha>0.40$. With only two exceptions, the DSDP and Bahamian samples, as well as the most dispersed SDS-treated AMB-1, have $\alpha$ between 0.25 and 0.30 . The most dispersed synthetic magnetite sample we produced had $\alpha=0.34$, and the trend from the most concentrated to the most dilute falls roughly upon a straight line in $\Delta B_{\mathrm{FWHM}}$ vs. $A$ space.

Although $\alpha$ is an empirical parameter, it serves as a proxy for the physically meaningful Gaussian linewidth $\sigma$. In the domain of parameter space occupied by magnetotactic bacteria and magnetofossils, $\alpha$ of synthetic spectra varies almost linearly with $\sigma$, and for most values of $B_{\text {an }}, \alpha$ increases monotonically as $\sigma$ increases (Fig. 10). The intact magnetotactic bacteria have nearly constant $\alpha$ values, which suggests that the differences among their spectra are predominantly caused by variations of $B_{\mathrm{an}}$. The $B_{\text {an }}$ and $\sigma$ parameters obtained by fitting the bacterial and synthetic samples (Tables 1 and 2) support this hypothesis; the intact and ultrasonicated AMB-1 have $\sigma \approx 30 \mathrm{mT}$, while SDS-treated AMB-1 have $\sigma \approx 50 \mathrm{mT}$ and the synthetic magnetite powders have $\sigma \geq 64 \mathrm{mT}$. We propose that $\alpha<0.30$ suggests the presence of magnetofossils, with lower $\alpha$ values reflecting more intact chains.

At the moment, the data are not strong enough to support firm claims about the presence or absence of biogenic magnetite based on ferromagnetic resonance

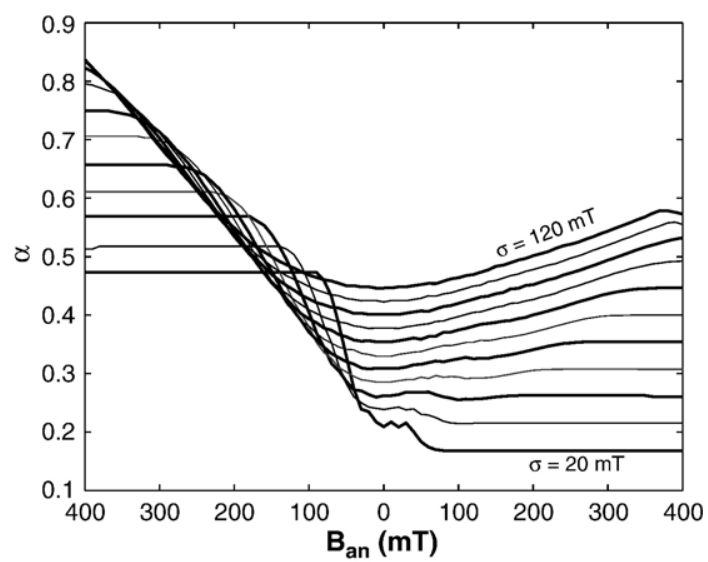

Fig. 10. The factor $\alpha$ for model spectra. $\alpha$ is plotted for synthetic spectra with first-order uniaxial anisotropy specified by $B_{\text {an }}$. Contour lines show values of $\sigma$ ranging from $20 \mathrm{mT}$ to $120 \mathrm{mT}$ in $10 \mathrm{mT}$ steps. In the region occupied by measured magneotactic bacteria and magnetofossil-bearing samples ( $B_{\text {an }} \geq 75 \mathrm{mT}, \alpha<0.30$ ), $\alpha$ varies nearly linearly with $\sigma$. spectra alone, except perhaps in the case of bacteria that have undergone minimal diagenetic alteration. Nonetheless, FMR provides a useful guide in the search for magnetofossils, and in sediments in which biogenic magnetite is known to be a major contributor to magnetization, FMR allows rapid assessment of the degree to which the magnetite chains have been disrupted. Its speed may also make it a useful tool in unmixing the magnetic components of sediments, although further work in this area is needed. Furthermore, as TEM of sedimentary biogenic magnetite requires the extraction of magnetite from the sediments, FMR provides the only known technique for detecting the presence of linear arrangements of magnetite crystals in situ.

\section{Conclusions}

1. Linear arrangements of magnetite crystals generally yield FMR spectra with $g_{\text {eff }}<2.12$ and $A<1$. To date, all intact magnetosome chains and almost all sedimentary samples believed to have a significant magnetofossil component fall within this domain, as do physically generated magnetite strings produced by dilution of lysed bacterial magnetite or synthetic magnetite.

2. Intact, biogenic magnetite chains yield derivative spectra with multiple low-field local maxima because their constituent particles experience fairly uniform magnetic anisotropy. Physically generated magnetite strings, which have more heterogeneous magnetic anisotropy, do not exhibit multiple local maxima.

3. Among the magnetotactic bacteria, greater crystal anisotropy produces both higher $\Delta B_{\mathrm{FWHM}}$ and lower $A$. MV-1, which produces chains of elongate truncated hexa-octahedral crystals, and MS-1 and AMB-1, which produce chains of cubo-octahedral crystals, have nearly identical values of $\alpha=0.17 \mathrm{~A}+$ $9.8 \times 10^{-4} \Delta B_{\mathrm{FWHM}} / \mathrm{mT}$, which is an empirical proxy for symmetric spectral broadening caused by factors such as sample heterogeneity. All magnetotactic bacteria measured have $\alpha<0.25$, while almost all sedimentary samples believed to contain significant biogenic magnetite have $\alpha$ between 0.25 and 0.30 . We suggest that, in samples containing magnetofossils, increasing $\alpha$ reflects a lower magnetofossil content and/or increased diagenetic alteration.

4. FMR provides a powerful tool for searching for samples likely to contain magnetofossils and for assessing the degree of alteration of magnetofossils in samples known to contain them. 


\section{Acknowledgements}

We thank J. Grotzinger for access to the Bahamian samples, J. Ewing for field assistance in the Bahamas, G. Rossman, S. Kim, T. Raub, and two anonymous reviewers for helpful discussion, B. Brunschweig and A. Di Bilio for technical assistance with the MPMS and EPR spectrometer, respectively, and the Beckman Institute for funding the use of the MPMS. REK was supported by a NSF Graduate Research Fellowship and a Moore Foundation Fellowship, BPW by the NASA Mars Fundamental Research and NSF Geophysics Programs, ACM and CZN by the Agouron Institute, HV by the Natural Sciences and Engineering Research Council of Canada, and JLK by NASA Exobiology grant NAG5-10618 and the NASA Astrobiology Institute.

\section{References}

[1] R.E. Kopp, C.Z. Nash, J.L. Kirschvink, J.R. Leadbetter, A possible magnetite/maghemite electrochemical battery in the magnetotactic bacteria, Eos 85 (2004) (Abst. GP34A-06).

[2] S.L. Simmons, D.A. Bazylinski, K.J. Edwards, South-seeking magnetotactic bacteria in the northern hemisphere, Science 311 (2006) 371-374.

[3] J.L. Kirschvink, South-seeking magnetic bacteria, J. Exp. Biol. 86 (1980) 345-347.

[4] S.B.R. Chang, J.L. Kirschvink, Magnetofossils, the magnetization of sediments, and the evolution of magnetite biomineralization, Annu. Rev. Earth Planet. Sci. 17 (1989) 169-195.

[5] D.A. Bazylinski, B.M. Moskowitz, Microbial biomineralization of magnetic iron minerals: Microbiology, magnetism and environmental significance, Geomicrobiology: interactions between microbes and minerals, Rev. Miner. 35 (1997) 181-223.

[6] K.L. Thomas-Keprta, D.A. Bazylinski, J.L. Kirschvink, S.J. Clemett, D.S. McKay, S.J. Wentworth, H. Vali, E.K. Gibson, C.S. Romanek, Elongated prismatic magnetite crystals in ALH84001 carbonate globules: potential Martian magnetofossils, Geochim. Cosmochim. Acta 64 (2000) 4049-4081.

[7] A.P. Taylor, J.C. Barry, R.I. Webb, Structural and morphological anomalies in magnetosomes: possible biogenic origin for magnetite in ALH84001, J. Microsc. 201 (2001) 84-106.

[8] J.L. Kirschvink, A seventh criterion for the identification of bacterial magnetofossils, Eos 82 (2001) S131.

[9] A. Witt, K. Fabian, U. Bleil, Three-dimensional micromagnetic calculations for naturally shaped magnetite: octahedra and magnetosomes, Earth Planet. Sci. Lett. 233 (2005) 311-324.

[10] T. Yamazaki, H. Kawahata, Organic carbon flux controls the morphology of magnetofossils in marine sediments, Geology 26 (1998) 1064-1066.

[11] R. Egli, Characterization of individual rock magnetic components by analysis of remanence curves: 3 . Bacterial magnetite and natural processes in lakes, Phys. Chem. Earth 29 (2004) 869-884.

[12] P. Montgomery, E.A. Hailwood, A.S. Gale, J.A. Burnett, The magnetostratigraphy of Coniacian late Campanian chalk sequences in southern England, Earth Planet Sci. Lett. 156 (1998) 209-224.
[13] S.B.R. Chang, J.F. Stolz, J.L. Kirschvink, S.M. Awramik, Biogenic magnetite in stromatolites: 2. Occurrence In ancient sedimentary environments, Precambrian Res. 43 (1989) $305-315$.

[14] R. Egli, Analysis of the field dependence of remanent magnetization curves, J. Geophys. Res. 108 (2003).

[15] R. Egli, Characterization of individual rock magnetic components by analysis of remanence curves: 1 . Unmixing natural sediments, Stud. Geophys. Geod. 48 (2004) 391-446.

[16] B.M. Moskowitz, R.B. Frankel, D.A. Bazylinski, Rock magnetic criteria for the detection of biogenic magnetite, Earth Planet. Sci Lett. 120 (1993) 283-300.

[17] C. Kittel, On the theory of ferromagnetic resonance absorption, Phys. Rev. 73 (1948) 155-161.

[18] E. Schlömann, Ferromagnetic resonance in polycrystal ferrites with large anisotropy: general theory and application to cubic materials with a negative anisotropy constant, J. Phys. Chem. Solids 6 (1958) 257-266.

[19] A.H. Morrish, The Physical Principles of Magnetism, J. Wiley, New York, 1965.

[20] B.P. Weiss, S.S. Kim, J.L. Kirschvink, R.E. Kopp, M. Sankaran, A. Kobayashi, A. Komeili, Ferromagnetic resonance and low temperature magnetic tests for biogenic magnetite, Earth Planet. Sci. Lett. 224 (2004) 73-89.

[21] G. Calas, Electron paramagnetic resonance, Rev. Miner. 18 (1988) 513-571.

[22] L.R. Bickford Jr., Ferromagnetic resonance absorption in magnetite single crystals, Phys. Rev. 78 (1950) 449-457.

[23] A. Komeili, H. Vali, T.J. Beveridge, D.K. Newman, Magnetosome vesicles are present before magnetite formation, and MamA is required for their activation, Proc. Natl. Acad. Sci.U. S. A. 101 (2004) 3839-3844.

[24] Y.A. Gorby, T.J. Beveridge, R.P. Blakemore, Characterization of the bacterial magnetosome membrane, J. Bacteriol. 170 (1988) 834-841.

[25] A. Kobayashi, J.L. Kirschvink, C.Z. Nash, R.E. Kopp, D.A. Sauer, L.E. Bertani, W.F. Voorhout, T. Taguchi, Experimental observation of magnetosome chain collapse in magnetotactic bacteria: sedimentological, paleomagnetic, and evolutionary implications, Earth Planet. Sci. Lett. 245 (2006) 538-550.

[26] A.P. Philipse, D. Maas, Magnetic colloids from magnetotactic bacteria: chain formation and colloidal stability, Langmuir 18 (2002) 9977-9984.

[27] L.A. Hardie, Sedimentation on the Modern Carbonate Tidal Flats of Northwest Andros Island, Bahamas, Johns Hopkins University Press, Baltimore, 1977, 202 pp.

[28] K.J. Hsü, J.L. LaBrecque, M.F. Carman, A.M. Gombos, A. Karpoff, J.A. McKenzie, S.F. Percival, N.P. Petersen, K.A Pisciotto, R.Z. Poore, E. Schreiber, L. Tauxe, P. Tucker, H.J. Weissert, Initial Reports of the Deep Sea Drilling Project, vol 73, U.S. Govt. Printing Office, Washington, 1984, 798 pp.

[29] H. Vali, J.L. Kirschvink, Magnetofossil dissolution in a paleomagnetically unstable deep-sea sediment, Nature 339 (1989) 203-206.

[30] C.R. Denham, A. Cox, Evidence that the Laschamp polarity event did not occur 13,300-30,400 years ago, Earth Planet. Sci Lett. 13 (1971) 181-190.

[31] R. Egli, W. Lowrie, Anhysteretic remanent magnetization of fine magnetic particles, J. Geophys. Res. 107 (2002).

[32] S. Cisowski, Interacting vs. non-interacting single-domain behavior in natural and synthetic samples, Phys. Earth Planet. Inter. 26 (1981) 56-62. 
[33] E.S. Keeping, Introduction to Statistical Inference, Dover, New York, 1995, $451 \mathrm{pp}$.

[34] D.L. Griscom, Ferromagnetic resonance spectra of lunar fines: some implications of line shape analysis, Geochim. Cosmochim. Acta 38 (1974) 1509-1519.

[35] D.L. Griscom, Ferromagnetic resonance condition and powder pattern analysis for dilute, spherical, single-domain particles of cubic crystal structure, J. Magn. Res. 45 (1981) 81-87.

[36] L.E. Drain, The broadening of magnetic resonance lines due to field inhomogeneities in powdered samples, Proc. Phys. Soc. 80 (1962) 1380-1382.

[37] U. Zeile, Fundamentals of cryo preparation and replica technique, 7th Asia-Pacific Electron Microscopy Conference, Singapore, 2000, pp. 342-357.

[38] R.F. Butler, S.K. Banerjee, Theoretical single-domain grain size range in magnetite and titanomagnetite, J. Geophys. Res. 80 (1975) 4049-4058.

[39] J.C. Diaz Ricci, J.L. Kirschvink, Magnetic domain state and coercivity predictions for biogenic greigite (Fe3S4): a compari- sion of theory with magnetosome observations, Geophys. J. Res. 97 (1992) 17309-17315.

[40] A.R. Muxworthy, E. McClellan, Review of the low-temperature magnetic properties of magnetite from a rock magnetic perspective, Geophys. J. Int. 140 (2000) 101-114.

[41] K. Abe, Y. Miyamoto, S. Chikazumi, Magnetocrystalline anisotropy of low-temperature phase of magnetite, J. Phys. Soc. Jpn. 41 (1976) 1894-1902.

[42] J.G. King, W. Williams, Low-temperature magnetic properties of magnetite, J. Geophys. Res. 105 (2000) 16427-16436.

[43] D.F. McNeill, Biogenic magnetite from surface Holocene carbonate sediments, Great Bahama Bank, J. Geophys. Res. 95 (1990) 4363-4371.

[44] D.F. McNeill, R.N. Ginsburg, S.B.R. Chang, J.L. Kirschvink, Magnetostratigraphic dating of shallow-water carbonates from San-Salvador, Bahamas, Geology 16 (1988) 8-12.

[45] D.F. McNeill, J.L. Kirschvink, Early dolomitization of platform carbonates and the preservation of magnetic polarity, J. Geophys. Res. 98 (1993) 7977-7986. 


\title{
The Paleoproterozoic snowball Earth: A climate disaster triggered by the evolution of oxygenic photosynthesis
}

\author{
Robert E. Kopp*, Joseph L. Kirschvink, Isaac A. Hilburn, and Cody Z. Nash \\ Division of Geological and Planetary Sciences, California Institute of Technology 170-25, Pasadena, CA 91125
}

Communicated by Paul F. Hoffman, Harvard University, Cambridge, MA, June 14, 2005 (received for review April 8, 2004)

\begin{abstract}
Although biomarker, trace element, and isotopic evidence have been used to claim that oxygenic photosynthesis evolved by 2.8 giga-annum before present ( $\mathrm{Ga}$ ) and perhaps as early as $3.7 \mathrm{Ga}$, a skeptical examination raises considerable doubt about the presence of oxygen producers at these times. Geological features suggestive of oxygen, such as red beds, lateritic paleosols, and the return of sedimentary sulfate deposits after $a \approx 900$-million year hiatus, occur shortly before the $\approx 2.3-2.2$ Ga Makganyene "snowball Earth" (global glaciation). The massive deposition of $\mathrm{Mn}$, which has a high redox potential, practically requires the presence of environmental oxygen after the snowball. New age constraints from the Transvaal Supergroup of South Africa suggest that all three glaciations in the Huronian Supergroup of Canada predate the Snowball event. A simple cyanobacterial growth model incorporating the range of $\mathrm{C}, \mathrm{Fe}$, and $\mathrm{P}$ fluxes expected during a partial glaciation in an anoxic world with high-Fe oceans indicates that oxygenic photosynthesis could have destroyed a methane greenhouse and triggered a snowball event on timescales as short as 1 million years. As the geological evidence requiring oxygen does not appear during the Pongola glaciation at 2.9 Ga or during the Huronian glaciations, we argue that oxygenic cyanobacteria evolved and radiated shortly before the Makganyene snowball.
\end{abstract}

oxygen | Makganyene glaciation | Huronian glaciations | cyanobacteria

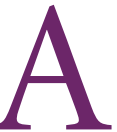

fter the rise of life itself, the most radical transformation of Earth's biogeochemical cycles occurred in the transition from an anoxic to an oxic world. This transformation took place in three phases. First, oxygenic photosynthesis evolved and brought into the world locally oxic environments. Second, oxygen became a major component of the atmosphere; some authors $(1,2)$ have suggested that this period was a protracted phase during which the ocean became euxinic. Finally, the whole ocean-atmosphere system took on its modern oxygendominated cast.

Although the timing of and relationship between the three stages have been topics of active research for many decades, there is still a wide divergence of opinion. Evidence from organic biomarkers (3-5) and arguments concerning trace element mobility (6) and biological productivity (7) have convinced many that $\mathrm{O}_{2}$-generating cyanobacteria and aerobic eukaryotes evolved no later than $\approx 2.78$ giga-annum before present $(\mathrm{Ga})$ and perhaps as long ago as $3.7 \mathrm{Ga}$. Meanwhile, the developing record of mass-independent fractionation (MIF) of sulfur isotopes in the sedimentary record, as well as some other geochemical tracers, has been interpreted as supporting a protracted atmospheric oxygenation over the period of $\approx 2.5-2.2 \mathrm{Ga}(8)$.

An early origin for oxygenic photosynthesis demands an explanation of how surface oxidation was muted for perhaps as long as 1,500 million years (My), until cyanobacteria finally surmounted some geochemical, environmental, or ecological barrier and successfully oxidized the planet. Perhaps this scenario is correct, and some abiotic change, such as the long-term escape of hydrogen to space (9), was the direct cause of planetary oxygenation. We suggest, however, that the data are also consistent with scenarios without oxygenic photosynthesis in the Archean. Herein we discuss an alternate hypothesis, one in which the evolution of cyanobacteria destroyed a methane greenhouse and thereby directly and rapidly triggered a planetary-scale glaciation, the ₹2.3-2.2 Ga Makganeyene "snowball Earth."

\section{Geological Setting}

The earliest evidence for glaciation comes from the late Archean and early Proterozoic, which suggests Earth at this time experienced global temperatures not much different from those today. The oldest known midlatitude glaciation, recorded in the Pongola Supergroup diamictite, occurred at $2.9 \mathrm{Ga}$ (10). The period from $2.45 \mathrm{Ga}$ until some point before $2.22 \mathrm{Ga}$ saw a series of three glaciations recorded in the Huronian Supergroup of Canada (11) (Fig. 1). The final glaciation in the Huronian, the Gowganda, is overlain by several kilometers of sediments in the Lorrain, Gordon Lake, and Bar River formations (Fms.). The entire sequence is penetrated by the 2.22 Ga Nipissing diabase (12); the Gowganda Fm. is therefore significantly older than $2.22 \mathrm{Ga}$.

In its eastern domain, the Transvaal Supergroup of South Africa contains two glacial diamictites, in the Duitschland and Boshoek Fms. The base of the Timeball Hill Fm., which underlies the Boshoek Fm., has a Re-Os date of 2,316 $\pm 7 \mathrm{My}$ ago (13). The Boshoek Fm. correlates with the Makganyene diamictite in the western domain of the Transvaal Basin, the Griqualand West region. The Makganyene diamictite interfingers with the overlying Ongeluk flood basalts, which are correlative to the Hekpoort volcanics in the eastern domain and have a paleolatitude of $11^{\circ} \pm 5^{\circ}(14)$. In its upper few meters, the Makganyene diamictite also contains basaltic andesite clasts, interpreted as being clasts of the Ongeluk volcanics. The low paleolatitude of the Ongeluk volcanics implies that the glaciation recorded in the Makganyene and Boshoek Fms. was planetary in extent: a snowball Earth event (15). Consistent with earlier whole-rock $\mathrm{Pb}-\mathrm{Pb}$ measurements of the Ongeluk Fm. (16), the Hekpoort Fm. contains detrital zircons as young as 2,225 \pm 3 My ago (17), an age nearly identical to that of the Nipissing diabase in the Huronian Supergroup. As the Makganyene glaciation begins some time after $2.32 \mathrm{Ga}$ and ends at $2.22 \mathrm{Ga}$, the three Huronian glaciations predate the Makganyene snowball.

In contrast to the Makganyene Fm., the three Huronian diamictites are unconstrained in latitude. Poles from the Matachewan dyke swarm, at the base of the Huronian sequence, do indicate a latitude of $\approx 5.5^{\circ}(18)$, but $\approx 2 \mathrm{~km}$ of sedimentary deposits separate the base of the Huronian from the first glacial unit (19), which makes it difficult to draw conclusions about the latitude of the glacial units based on these poles.

Abbreviations: Ga, giga-annum before present; My, million years; BIF, banded iron formation; Fm., formation; MIF, mass-independent fractionation.

*To whom correspondence should be addressed. E-mail: rkopp@caltech.edu.

(c) 2005 by The National Academy of Sciences of the USA 


\section{Griqualand West}

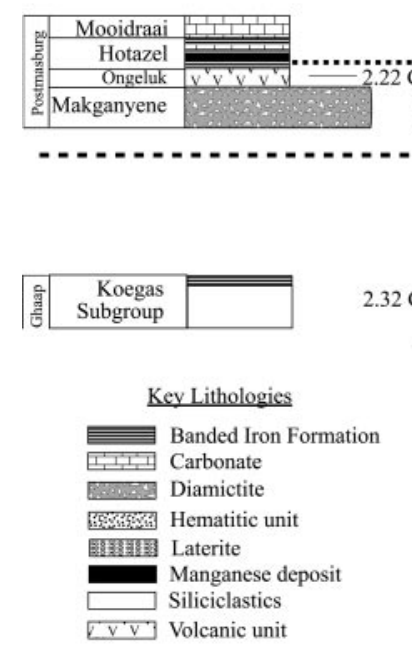

Eastern Transvaal

(Pretoria Group)
Huronian

Nipissing Diabase (2.22-2.21 Ga)

Fig. 1. Proposed correlation of the Huronian Supergroup and the upper Transvaal Supergroup. The three Huronian glacial units, penetrated and capped by the Nipissing diabase, predate the Makganyene diamictite in the Transvaal. The uppermost Huronian glacial unit, the Gowganda Fm., is overlain by hematitic units, perhaps reflecting a rise in $\mathrm{O}_{2}$. The basal Timeball Hill Fm. contains pyrite with minimal MIF (26), whereas the upper Timeball Hill Fm., which we suggest is correlative to the Lorrain or Bar River Fms., contains red beds. The Makganyene diamictite records a low-latitude, snowball glaciation (29), perhaps triggered by the destruction of $\mathrm{CH}_{4}$ greenhouse. It is overlain by the Kalahari Mn Field in the Hotazel Fm., the deposition of which requires free $\mathrm{O}_{2}$. Transvaal stratigraphy is based on ref. 17; Huronian stratigraphy is based on ref. 19.

Low latitude poles in the Lorrain Fm. $(20,21)$, which conformably overlies the Gowganda diamictite, are postdepositional overprints (22).

\section{MIF of Sulfur}

The recent discovery of MIF of S isotopes in Archean and early Proterozoic rocks has provided a major constraint on atmospheric $\mathrm{O}_{2}$. Large MIF of S (up to several hundred permil) is produced by photolysis of $\mathrm{SO}_{2}$ to $\mathrm{S}$ by light of wavelengths of $<200 \mathrm{~nm}$, which would have been unable to penetrate to the lower atmosphere had $\mathrm{O}_{2}$ levels been above a few percent of the present atmospheric level (23). To preserve MIF, multiple atmospheric $S$ species must be maintained as partially isolated reservoirs rather than being homogenized by oxidation of reduced species like hydrogen sulfide and polysulfur, as would occur at greater than $\approx 10^{-5}$ present atmospheric level $\mathrm{O}_{2}$ (24). Mixing and dilution of the atmospherically fractionated component, both in the atmosphere and in the oceans, presumably yields the observed Archean MIF values of up to $\approx 10 \%$ (25). An active oceanic S cycle, as would exist at moderately high $\mathrm{O}_{2}$ levels, also would likely prevent the preservation of MIF.

Before the deposition of the $\approx 2.32$ Ga Rooihogite and Timeball Hill Fms. in the Transvaal Supergroup $(13,26)$ and the McKim Fm. in the Huronian Supergroup ${ }^{\dagger}$ sedimentary sulfides often display MIF $(8,26,27)$ over an order of magnitude larger than the largest values observed in modern sulfate aerosols (25, 28). One plausible interpretation of the diminished MIF observed in the Rooihogite, Timeball Hill, and McKim Fms. is a rise in atmospheric $\mathrm{O}_{2}$. At present, only glaciers record the MIF sometimes present in sulfate aerosols; in the marine environment, with riverine input composing $\approx 90 \%$ of sulfate input and aerosols composing $\approx 10 \%$, MIF is not preserved (25). The small range of MIF could reflect an environment in which atmospheric chemistry began to approach modern conditions, decreasing the magnitude of MIF, but in which aerosols formed the major component of marine sulfate input, allowing its preservation.

The small range of MIF also permits an opposite interpretation. Rather than a decrease in atmospheric fractionation, the diminution could be a product of increased continental input and ocean/ atmosphere mixing driven by glacial conditions. The period of 2.5-2.2 Ga was a time of glaciations. Enhanced glacial weathering could have made unfractionated $\mathrm{S}$ from igneous sources a larger

tWing, B. A., Brabson, E., Farquhar, J., Kaufman, A. J., Rumble, D., III, \& Bekker, A. (2002) Geochim. Cosmochim. Acta 68, A840 (abstr.). part of the marine $S$ budget, whereas sharper thermal gradients would have driven homogenization of the S pool. Both effects would have decreased sedimentary MIF. If this interpretation is correct, only the final decrease of MIF after $\approx 2.09$ Ga may reflect the rise of atmospheric $\mathrm{O}_{2}$, and the oxygenation event may have been more rapid than the length of Farquhar and Wing's (8) MIF Stage II (2.45-2.09 Ga) indicates.

\section{Appearance of Local $\mathbf{O}_{2}$}

Both the Huronian and the Transvaal Supergroups contain features suggestive of, but not demanding, the first appearance of $\mathrm{O}_{2}$ in the period between the Gowganda and Makganyene glaciations. As noted in ref. 8, MIF in the Huronian rocks examined so far is at diminished values compatible with either increased atmospheric $\mathrm{O}_{2}$ or enhanced glacial weathering and ocean/atmosphere mixing. The Lorrain Fm. contains red beds, as well as a hematitic paleosol at VilleMarie, Quebec, and the Bar River Fm. contains pseudomorphs after gypsum, consistent with an increase in sulfate levels from oxidative weathering of sulfide minerals on land. In the Transvaal Supergroup, the upper Timeball Hill Fm., like the Lorrain Fm., contains red beds.

Stronger evidence for $\mathrm{O}_{2}$ appears after the Makganyene glaciation. The Ongeluk Fm. is overlain by the Hotazel Fm., which consists predominantly of banded iron formation (BIF). The basal half meter of the Hotazel Fm. contains dropstones (29) (see Supporting Text, which is published as supporting information on the PNAS web site), which suggests it was deposited toward the end of the glacial period. The Hotazel Fm. hosts a massive Mn member (29): a blanket of $\mathrm{Mn}$ deposition unmatched by any other known in the world, $\approx 50 \mathrm{~m}$ thick and with an erosional remnant, the Kalahari Mn Field, measuring $\approx 11 \times 50$ $\mathrm{km}$ in extent (30).

The Kalahari Mn Field indicates the release of large quantities of $\mathrm{O}_{2}$ into the ocean and therefore suggests a highly active postglacial aerobic biosphere. In seawater at circumneutral $\mathrm{pH}$, only $\mathrm{NO}_{3}{ }^{-}$and $\mathrm{O}_{2}$ can chemically oxidize soluble $\mathrm{Mn}^{2+}$ to produce insoluble Mn(IV) (31) (Table 1). Although a $\mathrm{Mn}^{2+}$ oxidizing phototroph also could produce $\mathrm{Mn}(\mathrm{IV})$, no such organism has ever been identified. Given the high redox potential of the $\mathrm{Mn}(\mathrm{IV}) / \mathrm{Mn}(\mathrm{II})$ couplet, it is likely that no such organism exists. The unexcited $\mathrm{P}_{870}$ reaction center in purple bacteria has a redox potential too low to accept electrons from $\mathrm{Mn}(\mathrm{II})$ (Table 1), but no known photosystem reaction center aside from 
Table 1. Midpoint potentials of relevant redox couplets

\begin{tabular}{lrr} 
& \multicolumn{2}{c}{$\mathrm{E}, \mathrm{mV}$} \\
\cline { 2 - 3 } Redox couplet & $\mathrm{pH} 7$ & $\mathrm{pH} 8$ \\
\hline $\mathrm{CO}_{2} / \mathrm{CH}_{4}{ }^{*+}$ & -230 & -289 \\
$\mathrm{SO}_{4}^{2-} / \mathrm{HS}^{-\ddagger}$ & -217 & -284 \\
$\mathrm{Fe}(\mathrm{OH})_{3}$ (ferrihydrite) $/ \mathrm{Fe}^{2+\ddagger}$ & -5 & -183 \\
$\mathrm{UO}_{2}\left(\mathrm{CO}_{3}\right)_{2}^{2-} / \mathrm{UO}_{2}$ (uraninite) & -18 & -137 \\
$\quad+\mathrm{CO}_{2}^{* \S}$ & & \\
$\mathrm{NO}_{3}^{-} / \mathrm{NH}_{4}^{+\ddagger}$ & +366 & +292 \\
$\mathrm{P}_{870} / \mathrm{P}_{870}^{+}$(purple bacteria) & $\approx+450$ & \\
$\mathrm{MnO}_{2}$ (pyrolusite) $/ \mathrm{Mn}^{2+\ddagger}$ & +490 & +372 \\
$\mathrm{NO}_{3}^{-} / \mathrm{N}_{2}{ }^{\ddagger}$ & +717 & +646 \\
$\mathrm{O}_{2} / \mathrm{H}_{2} \mathrm{O}$ & +815 & +756 \\
$\mathrm{P}_{680} / \mathrm{P}_{680}^{+}$ & $\approx+1,100$ & \\
$\quad($ Photosystem II) & & \\
\hline
\end{tabular}

All reactants not specifically noted are at standard state. Thermodynamic constants are from refs. 76 and 77. Photosystem potentials are from ref. 78.

*Calculated for $\mathrm{pCO}_{2}=100 \mathrm{mbar}$

${ }^{+}$Calculated for $\mathrm{pCH}_{4}=1$ mbar.

${ }^{\ddagger}$ Calculated with all aqueous reactants at $1 \mathrm{mM}$.

${ }^{5}$ Calculated with dissolved $\mathrm{U}$ species at $20 \mathrm{nM}$.

the $\mathrm{P}_{670}$ reaction center of photosystem II has a higher redox potential. Photosynthetic reduction of $\mathrm{Mn}(\mathrm{IV})$ therefore would likely require a two-part photosystem akin to that involved in oxygenic photosynthesis.

One plausible interpretation of the sequence of events leading up to the Paleoproterozoic snowball Earth is shown in Fig. 1 and is as follows.

1. Three glaciogenic units were deposited in the Huronian. The extent of the glaciations is not constrained, but they generally lack the lithographic features associated with snowball Earths, such as a sharp transition from a diamictite to a cap carbonate (32). Although the Espanola carbonate (33) could be a cap for the Bruce glaciation, without paleomagnetic data or additional lithographic features, its presence alone is insufficient to conclude that the Bruce glaciation was a snowball event.

2. Some of the earliest continental red beds were deposited in the Firstbrook member of the Gowganda Fm. and in the Lorrain and Bar River Fms. in Canada, as well as in the upper Timeball Hill Fm. in South Africa. The basal Timeball Hill Fm. has recently been dated at 2,316 $\pm 7 \mathrm{My}$ ago (13). In our proposed correlation, all of the red bed-bearing units were deposited after the last Huronian glaciation and before the Makganyene glaciation. The formation of the red beds could involve local $\mathrm{O}_{2}$, although it does not demand it (34). Syngenetic pyrite from the basal
Timeball Hall Fm. shows only slight MIF of S (26), consistent with the initiation of planetary oxygenation or enhanced glacial activity.

3. The low-latitude glaciation that formed the Makganyene and Boshoek diamictites (14) was initiated when the production of $\mathrm{O}_{2}$ triggered the collapse of a methane greenhouse. Although Pavlov et al. (35) proposed a similar trigger, they assumed a delay of at least $\approx 400$ My between the onset of oxygenic photosynthesis and its surficial expression. We suggest a more immediate linkage.

4. The Nipissing diabase intruded into the Huronian sequence (36), and the Ongeluk and Hekpoort volcanics were deposited (16).

5. The Hotazel Fm., which includes BIF and Mn members, was deposited in oxygenated waters in the aftermath of the snowball.

6. The upper siderite facies of the Hotazel Fm. and perhaps also part of the overlying Mooidrai dolomite were deposited as cap carbonates in the process of removing $\mathrm{CO}_{2}$ from a thick postsnowball greenhouse (29).

\section{Timescale for Methane Greenhouse Collapse}

For cyanobacteria to be directly responsible for triggering a planetary glaciation, they must have been able to produce enough $\mathrm{O}_{2}$ to destroy the methane greenhouse before the carbonate-silicate weathering cycle could compensate. On sufficiently long timescales, global cooling would slow silicate weathering and carbonate precipitation, thereby allowing $\mathrm{CO}_{2}$ to build up in the atmosphere (37). The response time of the carbonatesilicate weathering cycle is generally estimated at $\approx 1$ My (38), although the time to replace the greenhouse capacity lost in a methane greenhouse collapse may be longer. To estimate the timescale for destruction of the methane greenhouse, we constructed a steady-state ocean biogeochemistry model based on the assumptions that biological productivity was controlled by $\mathrm{P}$ and $\mathrm{N}$ and that $\mathrm{Fe}(\mathrm{II})$ oxidation was the main inorganic $\mathrm{O}_{2}$ sink (see Supporting Text).

The critical $\mathrm{P} / \mathrm{Fe}$ flux ratio for net oxidation of the surface ocean increases with the burial rate of $\mathrm{C}$ (see Fig. $2 a$, which is published as supporting information on the PNAS web site). For a $\mathrm{P}$ flux similar to today's value of $\approx 8 \times$ $10^{10} \mathrm{~mol} / \mathrm{y}(39)$ and a burial fraction of $2 \times 10^{-2}$, similar to modern anoxic environments, the model results indicate that the critical value is $\approx 1 / 50$. The current ratio of $\mathrm{P}$ flux to hydrothermal $\mathrm{Fe}$ flux is $\approx 1 / 2$, whereas the ratio of $P$ flux to hydrothermal and terrigenous Fe flux is $\approx 1 / 64$ (1). Rare earth element patterns of BIFs (40) and the stability of terrestrial ferrous sulfides in an anoxic atmosphere suggest that the main source of reactive $\mathrm{Fe}$ in the Archean was hydrothermal, so the $\mathrm{P} / \mathrm{Fe}$ ratio may have been closer to $1 / 2$ than $1 / 64$.

When the $\mathrm{P} / \mathrm{Fe}$ ratio falls below the critical value, essentially all $\mathrm{O}_{2}$ produced is captured by $\mathrm{Fe}^{2+}$. Above the critical $\mathrm{P} / \mathrm{Fe}$ value, there is a net release of $\mathrm{O}_{2}$ (Fig. 2a). Other dissolved $\mathrm{O}_{2}$ sinks, such as $\mathrm{Mn}^{2+}, \mathrm{H}_{2}$, and $\mathrm{CH}_{4}$, will consume some $\mathrm{O}_{2}$, but hydrothermal fluxes of these reductants are up to an order of magnitude less than the flux of $\mathrm{Fe}^{2+}$ (41). At today's levels, these are capable of consuming $\approx 7 \times 10^{10} \mathrm{~mol}$ of $\mathrm{O}_{2}$ per $\mathrm{y}$, equivalent to $4 \times 10^{-10} \mathrm{bar} / \mathrm{y}$. Although the flux of $\mathrm{H}_{2} \mathrm{~S}$ from vent fluids in today's high sulfate oceans is comparable to that of $\mathrm{Fe}^{2+}$, in an ocean where $\mathrm{Fe}^{2+}$ is the main electron donor, $\mathrm{H}_{2} \mathrm{~S}$ levels would be lower. A formaldehyde rainout flux of $\approx 10^{12} \mathrm{~mol} / \mathrm{y}$ (24) would be the largest $\mathrm{O}_{2}$ sink, capable of adsorbing $\approx 6 \times 10^{-9}$ bar of $\mathrm{O}_{2}$ per $\mathrm{y}$, but would be nullified by $\mathrm{H}_{2} \mathrm{O}_{2}$ rainout once even a small amount of $\mathrm{O}_{2}$ accumulated in the atmosphere. $\mathrm{O}_{2}$ production in excess of $\approx 6 \times 10^{-9} \mathrm{bar} / \mathrm{y}(1 \mathrm{bar}=100$ $\mathrm{kPa}$ ) would therefore be sufficient to initiate $\mathrm{CH}_{4}$ oxidation, and, once begun, net $\mathrm{O}_{2}$ production in excess of $\approx 4 \times$ $10^{-10} \mathrm{bar} / \mathrm{y}$ would suffice to continue it. At the rates predicted by our model, destruction of a 1-mbar methane greenhouse, if it occurred at all, would likely occur within a few My, a timescale comparable with the carbonate-silicate weathering cycle. Therefore, either cyanobacteria did not evolve until shortly before the oxygenation event, or the nutrient flux did not reach sufficiently high levels at any point after the evolution of cyanobacteria until then.

If cyanobacteria were present during the Huronian glaciations, the increased $P$ flux into the oceans generated by glacial weathering (42) should have triggered the oxygenation event. Instead, the oxygenation event seems to correlate with the later Makganyene glaciation; this finding suggests the evolution of cyanobacteria occurred in the interval between the Huronian glaciations and the Makganyene glaciation.

Whether $\mathrm{N}$ limitation could have delayed the destruction of a methane greenhouse ${ }^{\ddagger}$ depends on the Fe demand of the $\mathrm{N}_{2}$ fixers and the ability of cyanobacteria to capture $\mathrm{Fe}$ before it reacted with $\mathrm{O}_{2}$ and sank beneath the photic zone as a ferric precipitate. With anoxic deep waters

FFalkowski, P. G., Follows, M. \& Fennel, K. (2003) EOS Trans. Am. Geophys. Union 84, Suppl., abstr. U52C-01. 
providing a large source of Fe to the photic zone, life for an early Proterozoic diazotroph would have been easier than for modern diazotrophs that must subsist off of $\mathrm{Fe}$ transported from the continents. $\mathrm{C} / \mathrm{Fe}$ ratios in $\mathrm{N}_{2}$-fixing populations of Trichodesmium range from $<2,000$ to 50,000 (43). If early cyanobacteria were inefficient at both capturing and using $\mathrm{Fe}$ (e.g., $\mathrm{C} / \mathrm{Fe}=2,000$; capture efficiency $=$ $1 \%$ ), then $\mathrm{N}$ limitation could have protected the methane greenhouse, but under more optimistic assumptions (either a higher capture efficiency or a higher $\mathrm{C} / \mathrm{Fe}$ ratio), it would not have done so (Fig. $2 b$ ). The geologic record, not computational models, must ultimately decide.

\section{Implications of the Possible Late Evolution of Cyanobacteria}

The interpretation presented here suggests that planetary oxygenation began in the interval between the end of the Huronian glaciations and the onset of the Makganyene glaciation and that the Paleoproterozoic snowball Earth was the direct result of a radical change in the biosphere. In the Archean and earliest Proterozoic oceans, life may have been fueled predominantly by $\mathrm{Fe}$, with $\mathrm{Fe}(\mathrm{II})$ used as the electron donor for photosynthesis and $\mathrm{Fe}(\mathrm{III})$ as the main electron acceptor for respiration. The sediments therefore would be moderately oxidizing and the surface waters reduced (34). Because the redox potential for $\mathrm{Mn}^{2+}$ oxidation is much higher than that of $\mathrm{Fe}^{2+}$, Mn would have to be removed from the oceans in reduced form. The carbonates precipitated at this time contain up to $\approx 2 \% \mathrm{Mn}(30)$, indicating that $\mathrm{Mn}^{2+}$ reached shallow waters and coprecipitated with $\mathrm{Ca}^{+2}$; oxidized $\mathrm{Mn}$ is extremely rare. The atmosphere was likely reducing. Astrophysical models predict the Sun was substantially dimmer than today, but a $\mathrm{CH}_{4}$ greenhouse (44) produced by methanogens living in a reduced upper ocean would have kept the planet warm enough to allow for the presence of liquid water without leading to massive siderite precipitation (45).

This world would have been overthrown when cyanobacteria capable of oxygenic photosynthesis evolved, which molecular phylogenies indicate occurred later than the main bacterial radiation (46). The surface waters became oxidizing and more productive. Reduced $\mathrm{C}$ accumulated in the sediments; methanogenesis moved from the surface ocean to the deep ocean, where it was isolated from the atmosphere. Methanotrophy in the ocean and photochemistry in the atmosphere used $\mathrm{O}_{2}$ to transform atmospheric $\mathrm{CH}_{4}$ to $\mathrm{CO}_{2}$, a less effective greenhouse gas. The rise of $\mathrm{O}_{2}$ thus might have triggered a glacial interval without the $\approx 400$ My delay assumed by others (35).

Phosphate flux into the oceans correlates with increased continental weathering during glacial intervals (42), so increased continental weathering produced by the glaciations may have increased nutrient availability above the threshold for net $\mathrm{O}_{2}$ release into the atmosphere. Because of the relatively low global temperature, it would have taken only a trace of $\mathrm{OH}$ radicals from the oxygenic bloom produced when cyanobacteria appeared in a high-P ocean to damage the $\mathrm{CH}_{4}$ greenhouse enough to bring average global temperatures to $<0^{\circ} \mathrm{C}$. During the at least $\approx 35$ My $(29,47)$ it took to build up a sufficient $\mathrm{CO}_{2}$ greenhouse to escape from the snowball, hydrothermal fluxes would have built up a rich supply of nutrients in the oceans. When the planet finally warmed, the oceans were ripe for a cyanobacterial bloom. The $\mathrm{O}_{2}$ produced by the bloom cleared out tens of My worth of accumulated reductants and thus produced the Kalahari Mn field (29).

\section{Biomarker Counterevidence for Archean $\mathbf{O}_{2}$}

Despite the parsimony of cyanobacterial evolution occurring within a few My before the onset of the Paleoproterozoic snowball Earth, some organic biomarker evidence and indirect sedimentological and geochemical arguments have been used to suggest that the origin of cyanobacteria dates to far earlier times: at least $2.78 \mathrm{Ga}$ and maybe as long ago as $3.7 \mathrm{Ga}$.

The critical piece of evidence placing the origin of cyanobacteria and locally oxic environments in the Archean is the discovery in bitumens from rocks as old as $2.78 \mathrm{Ga}$ of organic biomarkers apparently derived from lipids used by cyanobacteria and eukaryotes in their cell membranes. Although Brocks et al. (48) concluded that the bitumens were likely syngenetic, they could not exclude the possibility that they were postdepositional contaminants. Even if the biomarkers are as old as their host rocks, however, the uniformitarian extrapolation of modern lipid distributions to the Archean should be viewed cautiously.

Hopanes. Among modern organisms, 2-methyl-bacteriohopanepolyol is produced predominantly by cyanobacteria and in trace quantities by methylotrophs like Methylobacterium organophilum (3). Hopanes derived from 2-methylbacteriohopanepolyol can be preserved in sedimentary rocks, where they have been used as tracers for cyanobacteria (3). Hopanol synthesis has traditionally been assumed, based on the understood modern distribution of hopanols, to oc- cur only in aerobic organisms. Fischer et al. (79) recently demonstrated, however, that Geobacter sulfurreducens can synthesize diverse hopanols, although not 2-methyl-hopanols, when grown under strictly anaerobic conditions. Thus, fossil hopanes do not necessarily imply the presence of $\mathrm{O}_{2}$-producing organisms, and nothing about 2-methyl-hopanols suggests that they are any different in this respect. Archean 2-methyl-hopanes also might have been produced by ancestral cyanobacteria that predated oxygenic photosynthesis.

Steranes. Produced by eukaryotes for use in the cell membrane, sterols are preserved in the rock record as steranes. Brocks et al. $(4,5)$ recovered steranes, along with 2-methyl-bacteriohopanepolyol, from $2.78 \mathrm{Ga}$ Pilbara Craton shales. Because there is no known anaerobic sterol synthesis pathway, they used their discovery to argue for the presence of $\mathrm{O}_{2}$.

Cholesterol biosynthesis in modern organisms is a long biochemical pathway that employs the following four $\mathrm{O}_{2}$-dependent enzymes: (i) squalene epoxidase, (ii) lanosterol 14- $\alpha$-methyl demethylase cytochrome P450, (iii) sterol-4- $\alpha$-methyl oxidase, and (iv) lathosterol oxidase (49). These $\mathrm{O}_{2}$-dependent enzymes perform reactions that, although not currently known to occur biochemically in anaerobic organisms, could feasibly occur without $\mathrm{O}_{2}$. Moreover, the substitution of an $\mathrm{O}_{2}$-dependent enzyme for an anaerobic step in a biosynthetic pathway appears to be a common evolutionary occurrence. Raymond and Blankenship (50) found that, of the $473 \mathrm{O}_{2}$-dependent enzymatically catalyzed reactions in the BioCyc database (www.biocyc.org), 20 have at least one $\mathrm{O}_{2}$-independent counterpart that performs the same reaction. For instance, AcsF catalyzes an $\mathrm{O}_{2}$-dependent cyclization step in the synthesis of chlorophyll and bacteriochlorophyll, a pathway that must have existed before the evolution of oxygenic photosynthesis. The $\mathrm{O}_{2}$-independent enzyme BchE performs the same reaction as AcsF but uses vitamin $\mathrm{B}_{12}$ in place of $\mathrm{O}_{2}(50)$. The assumption that sterol synthesis is always $\mathrm{O}_{2}$-dependent and always has been therefore merits close inspection.

Indeed, the Hamersley bitumens include their own cautionary message about the application of uniformitarian assumptions to fossil lipids. Dinosterane is generally accepted to be characteristic of dinoflagellates and is interpreted as a dinoflagellate biomarker in Phanerozoic rocks (51). Yet even though an Archean origin for dinoflagellates seems implausible, because it would indicate the Ar- 
chean origin of modern eukaryotic group not known in the fossil record until at least the Paleozoic (52), Brocks et al. (5) found dinosterane. They interpreted the molecule as being produced by eukaryotes of unknown affinities, although an alternative explanation is that these modern-style putative Archean biomarkers are contaminants.

\section{Indirect Counterevidence for Archean $\mathbf{O}_{\mathbf{2}}$}

Although organic biomarkers may be difficult to interpret, they are a significant improvement on several other geological tracers that have been used to argue for the presence of cyanobacteria and environmental $\mathrm{O}_{2}$, including microfossils, stromatolites, BIFs, and assorted isotopic fractionations.

Microfossils. Before $2 \mathrm{Ga}$, when diversified assemblages with affinities to major groups of cyanobacteria first appear in the fossil record, the microfossil record is murky (53). Some have interpreted filamentous forms in earlier rocks as cyanobacterial remains (54-56), but Brasier et al. (57) recently questioned the biogenic nature of these objects. Moreover, cyanobacteria cannot be identified solely by a filamentous form. Many nonoxygenic bacteria are also filamentous, including some mat-forming green nonsulfur and purple sulfur bacteria $(58,59)$ and a methanogenic archeon (60). The wide variety of filamentous prokaryotes highlights a problem in identifying fossil microbes lacking clear evidence of cell differentiation based on morphology: Any given form has probably arisen many times in Earth history, both in extant and extinct organisms.

Stromatolites and BIFs. Two types of late Archean rock Fms. have often been interpreted as indicating cyanobacterial activity: stromatolites and BIFs. Des Marais (7) argued that large stromatolite reefs indicate the presence of cyanobacteria and therefore a locally aerobic environment, but large reefs also can form under anaerobic conditions. Populations of anaerobic methane oxidizers, for instance, have built massive reefs at methane seeps in the Black Sea (61). In addition, the Archean and Paleoproterozoic oceans were likely more supersaturated with respect to calcite and aragonite than the modern oceans (62), which would have facilitated the precipitation of large reefs even without biological participation. Indeed, abiotic processes may have played a major role in the formation of many Precambrian stromatolites (63). Moreover, although the deposition of ferric iron in BIFs has traditionally been taken to imply the presence of free $\mathrm{O}_{2}(40,64,65)$, BIFs also could have formed in a $\mathrm{O}_{2}$-free envi- ronment, either by photooxidation (66) or by $\mathrm{Fe}(\mathrm{II})$-oxidizing phototrophic bacteria $(67,68)$.

Isotopic Evidence. Rosing and Frei (6) argued based on isotopic evidence that $>3.7$ Ga metashale from West Greenland preserves signs of an aerobic ecosystem. They found organic C with $\delta^{13} \mathrm{C}$ values of $-25.6 \%$ in the same sediments as $\mathrm{Pb}$ with isotopic ratios indicating that the samples were originally enriched in $\mathrm{U}$ with respect to $\mathrm{Th}$ and interpreted this finding as reflecting an environment in which $U$ could be cycled between its reduced, insoluble U(IV) form and its oxidized, soluble U(VI) form. They concluded the light $\mathrm{C}$ was produced by oxygenic phototrophs and that biogenic $\mathrm{O}_{2}$ had permitted the redox cycling of U. However, all biological $\mathrm{C}$ fixation pathways and some abiotic mechanisms can produce light $\mathrm{C}$, and, just like the $\mathrm{Fe}(\mathrm{III})$ in BIFs, U(VI) can form in the absence of $\mathrm{O}_{2}$. At circumneutral $\mathrm{pH}$ values, the midpoint potential of the $\mathrm{U}(\mathrm{VI}) / \mathrm{U}(\mathrm{IV})$ couplet is $\approx 0$ $\mathrm{V}$, similar to the $\mathrm{Fe}(\mathrm{III}) / \mathrm{Fe}(\mathrm{II})$ couplet and considerably less oxidizing than $\mathrm{Mn}(\mathrm{IV}) / \mathrm{Mn}(\mathrm{II}), \mathrm{NO}_{3}{ }^{-} / \mathrm{N}_{2}$, or $\mathrm{O}_{2} / \mathrm{H}_{2} \mathrm{O}$ (see Table 1).

A strong negative $\delta^{13} \mathrm{C}$ excursion in organic $\mathrm{C}$ at $\approx 2.7 \mathrm{Ga}$ has been interpreted as evidence for the evolution of aerobic methanotrophy (69). Such light $\mathrm{C}$ suggests repeated fractionation: first in the production of $\mathrm{CH}_{4}$ subsequently oxidized to $\mathrm{CO}_{2}$, then in re-reduction by a primary producer; similar fractionations are observed today in environments with methanotrophs. But although $\mathrm{CH}_{4}$ oxidation was once thought to require $\mathrm{O}_{2}$, geochemical measurements (70) and recent microbiological work $(71,72)$ have demonstrated that $\mathrm{CH}_{4}$ oxidation also can occur with alternative electron acceptors, so $\mathrm{O}_{2}$ is not needed to explain the isotopic excursions. Although the anaerobic methane oxidizing bacteria studied today rely on sulfate, which is unlikely to have been available in a high-Fe Archean ocean, thermodynamics permits a variety of electron acceptors, including Fe(III), to be used for $\mathrm{CH}_{4}$ oxidation.

$\mathrm{S}$ isotope data indicate local spikes in sulfate concentration starting $\approx 3.45 \mathrm{Ga}$ (73, 74). Canfield et al. (75) argued that these spikes were produced in high- $\mathrm{O}_{2}$ environments. But given the low redox potential of the sulfide/sulfate couplet, local sulfate spikes can be explained by scenarios that do not involve $\mathrm{O}_{2}$. Moreover, sedimentary sulfate deposits, which disappear in the rock record after $\approx 3.2$ Ga (75), do not reappear until after the Huronian glaciations, which suggests that high sulfate conditions were rare.
None of these indirect lines of evidence necessitate oxygenic photosynthesis. The case for cyanobacteria and locally oxic environments existing before the disappearance of MIF of S isotopes and the massive deposition of $\mathrm{Mn}$ in the Kalahari Mn Field rests, at the moment, solely on steranes.

\section{Future Directions}

Our model for a Paleoproterozoic origin of cyanobacteria is testable by several methods. It suggests that sterols in Archean rocks, if they are original, were synthesized by anaerobic reactions. It therefore should be possible to find or engineer enzymes capable of synthesizing cholesterol under anaerobic, biochemically plausible conditions. In addition, a continuous biomarker record that stretches back from time periods when $\mathrm{O}_{2}$ is definitely present into the Archean might reveal transitions in community composition. Current work with samples from recent drilling programs targeting the late Archean and the Paleoproterozic has begun this task. An intensive search for biomarkers with definite relationships to metabolism, such as those derived from the pigment molecules of phototrophic bacteria, also would produce a more convincing record. A search for cyanobacterial or eukaryotic fossils that predate $2.0 \mathrm{Ga}$ yet have affinities to modern groups would complement the geochemical approaches.

With respect to the record of MIF of $\mathrm{S}$, the timeline we propose for a rapid oxygenation scenario suggests that decreased fractionation during the interval of the Huronian glaciations may be a byproduct of enhanced glacial weathering and ocean/atmosphere mixing. If this scenario is correct, a similar phenomenon should have occurred in association with the Pongola glaciation at $\approx 2.9 \mathrm{Ga}$. Investigation of the Huronian deposits where low-MIF $S$ is found should reveal these deposits to be sedimentologically immature, reflecting glacial input. Additionally, the syngenicity of the MIF values should be tested through techniques such as the paleomagnetic search for significantly postdepositional formation of sulfides.

Finally, our model predicts that the Makganyene snowball Earth was a singular event. Convincing paleomagnetic evidence (including positive syn-sedimentary field tests) that demonstrated the Huronian glaciations were low-latitude would contradict our model and instead support a protracted episode of planetary oxygenation with multiple snowball events not directly triggered by a singular event, the evolution of cyanobacteria. 


\section{Summary}

Because of the importance of the evolution of cyanobacteria and the planetary oxygenation event in Earth history, it is particularly useful to consider multiple working hypotheses about these events. We propose a model that takes a skeptical attitude toward the evidence for Archean cyanobacteria and a protracted early Proterozoic planetary oxygenation. In our alternative scenario, an evolutionary accident, the genesis of oxygenic

1. Canfield, D. E. (1998) Nature 396, 450-453.

2. Anbar, A. D. \& Knoll, A. H. (2002) Science 297, 1137-1142.

3. Summons, R. E., Jahnke, L. L., Hope, J. M. \& Logan, G. A. (1999) Nature 400, 554-557.

4. Brocks, J. J., Logan, G. A., Buick, R. \& Summons, R. E. (1999) Science 285, 1033-1036.

5. Brocks, J. J., Buick, R., Summons, R. E. \& Logan, G. A. (2003) Geochim. Cosmochim. Acta 67, 43214335.

6. Rosing, M. T. \& Frei, R. (2004) Earth Planet. Sci. Lett. 217, 237-244.

7. Des Marais, D. J. (2000) Science 289, 1703-1705

8. Farquhar, J. \& Wing, B. A. (2003) Earth Planet. Sci. Lett. 213, 1-13.

9. Catling, D. C., Zahnle, K. J. \& McKay, C. P. (2001) Science 293, 839-843.

10. Nhelko, N. (2004) D.Phil. thesis (Rand Afrikaans University, Johannesburg).

11. Young, G. M., von Brunn, V., Gold, D. J. C. \& Minter, W. E. L. (1998) J. Geol. 106, 523-538.

12. Corfu, F. \& Andrews, A. J. (1986) Can. J. Earth Sci. 23, 107-109.

13. Hannah, J. L., Bekker, A., Stein, H. J., Markey, R. J. \& Holland, H. D. (2004) Earth Planet. Sci. Lett. 225, 43-52.

14. Evans, D. A., Beukes, N. J. \& Kirschvink, J. L. (1997) Nature 386, 262-266.

15. Tajika, E. (2003) Earth Planet. Sci. Lett. 214, 443-453.

16. Cornell, D. H., Schutte, S. S. \& Eglington, B. L. (1996) Precambrian Res. 79, 101-123.

17. Dorland, H. (2004) D.Phil. thesis (Rand Afrikaans University, Johannesburg).

18. Bates, M. P. \& Halls, H. C. (1990) Can. J. Earth Sci. 27, 200-211.

19. Young, G. M., Long, D. G. F., Fedo, C. M. \& Nesbitt, H. W. (2001) Sediment. Geol. 141, 233254.

20. Williams, G. E. \& Schmidt, P. W. (1997) Earth Planet. Sci. Lett. 153, 157-169.

21. Schmidt, P. \& Williams, G. (1999) Earth Planet. Sci. Lett. 172, 273-285.

22. Hilburn, I. A., Kirschvink, J. L., Tajika, E., Tada, R., Hamano, Y. \& Yamamoto, S. (2005) Earth Planet. Sci. Lett. 232, 315-332.

23. Farquhar, J., Savarino, J., Airieau, S. \& Thiemens, M. H. (2001) J. Geophys. Res. Planets 106, 3282932839.

24. Pavlov, A. A. \& Kasting, J. F. (2002) Astrobiology 2, 27-41.

25. Ono, S., Eigenbrode, J. L., Pavlov, A. A., Kharecha, P., Rumble, D., Kasting, J. F. \& Freeman, K. H. (2003) Earth Planet. Sci. Lett. 213, 15-30.

26. Bekker, A., Holland, H. D., Wang, P.-L., Rumble, D., III, Stein, H. J., Hannah, J. L., Coetzee, L. L. \& Beukes, N. J. (2004) Nature 427, 117-120.

27. Farquhar, J., Bao, H. \& Thiemens, M. (2000) Science 289, 756-758. photosynthesis, triggered one of the world's worst climate disasters, the $\mathrm{Pa}$ leoproterozoic snowball Earth. Intensive investigation of the time period of the Paleoproterozoic glaciations may reveal whether a novel biological trait is capable of radically altering the world and nearly bringing an end to life on Earth.

We thank R. Adler, N. Beukes, R. Blankenship, J. Brocks, H. Dorland, A. Kappler, J. Kasting, A. Maloof, D. Newman, S. Ono, A.

28. Savarino, J., Romero, A., Cole-Dai, J., Bekki, S. \& Thiemens, M. H. (2003) Geophys. Res. Lett. 30, 2131.

29. Kirschvink, J. L., Gaidos, E. J., Bertani, L. E., Beukes, N. J., Gutzmer, J., Maepa, L. N. \& Steinberger, R. E. (2000) Proc. Natl. Acad. Sci. USA 97, 1400-1405.

30. Cairncross, B., Beukes, N. \& Gutzmer, J. (1997) The Manganese Adventure: The South African Manganese Fields (Associated Ore \& Metal Corp. Ltd., Johannesburg).

31. Gaidos, E. J., Nealson, K. H. \& Kirschvink, J. L. (1999) Science 284, 1631-1633.

32. Hoffman, P. F. \& Schrag, D. P. (2002) Terra Nova 14, 129-155.

33. Bernstein, L. \& Young, G. M. (1990) Can. J. Earth Sci. 27, 539-551.

34. Walker, J. C. G. (1987) Nature 329, 710-712.

35. Pavlov, A. A., Brown, L. L. \& Kasting, J. F. (2001) J. Geophys. Res. Planets 106, 23267-23287.

36. Noble, S. R. \& Lightfoot, P. C. (1992) Can. J. Earth Sci. 29, 1424-1429.

37. Walker, J. C. G., Hays, P. B. \& Kasting, J. F. (1981) J. Geophys. Res. Oceans Atmos. 86, 9776-9782.

38. Berner, R. A., Lasaga, A. C. \& Garrels, R. M. (1983) Am. J. Sci. 283, 641-683.

39. Bjerrum, C. J. \& Canfield, D. E. (2002) Nature 417, 159-162.

40. Klein, C. \& Beukes, N. J. (1992) in The Proterozoic Biosphere, ed. Klein, C. (Cambridge Univ. Press, Cambridge, U.K.), pp. 147-152.

41. Elderfield, H. \& Schultz, A. (1996) Annu. Rev. Earth Planet. Sci. 24, 191-224.

42. Föllmi, K. B. (1995) Geology 23, 503-506.

43. Kustka, A., Carpenter, E. J. \& Sanudo-Wilhelmy, S. A. (2002) Res. Microbiol. 153, 255-262.

44. Pavlov, A. A., Kasting, J. F., Brown, L. L., Rages, K. A. \& Freedman, R. (2000) J. Geophys. Res. Planets 105, 11981-11990.

45. Rye, R., Kuo, P. H. \& Holland, H. D. (1995) Nature 378, 603-605.

46. Olsen, G. J., Woese, C. R. \& Overbeek, R. (1994) J. Bacteriol. 176, 1-6.

47. Pierrehumbert, R. T. (2004) Nature 429, 646-649.

48. Brocks, J. J., Buick, R., Logan, G. A. \& Summons, R. E. (2003) Geochim. Cosmochim. Acta 67, 42894319.

49. Risley, J. M. (2002) J. Chem. Ed. 79, 377-384.

50. Raymond, J. \& Blankenship, R. E. (2004) Geobiology 2, 199-203.

51. Moldowan, J. M. \& Jacobson, S. R. (2000) Int. Geol. Rev. 42, 805-812.

52. Falkowski, P. G., Katz, M. E., Knoll, A. H., Quigg, A., Raven, J. A., Schofield, O. \& Taylor, F. J. R. (2004) Science 305, 354-360.

53. Amard, B. \& Bertrand-Sarfati, J. (1997) Precambrian. Res. 81, 197-221.

54. Awramik, S. M., Schopf, J. W. \& Walter, M. R. (1983) Precambrian. Res. 20, 357-374.
Sessions, D. Sumner, T. Raub, B. Weiss, and three anonymous reviewers for advice and discussion; R. Tada for help with fieldwork in the Huronian; A. Pretorius and Assmang Limited for access to Nchwaning Mine; and P. Hoffman for communicating this manuscript. This work was supported in part by the Agouron Institute and by a National Aeronautics and Space Administration Astrobiology Institute cooperative agreement with the University of Washington. R.E.K. was supported by a National Science Foundation Graduate Research Fellowship and a Moore Foundation Fellowship.

55. Schopf, J. W. \& Packer, B. M. (1987) Science 237, $70-73$.

56. Schopf, J. W. (1993) Science 260, 640-646.

57. Brasier, M. D., Green, O. R., Jephcoat, A. P., Kleppe, A. K., Van Kranendonk, M. J., Lindsay, J. F., Steele, A. \& Grassineau, N. V. (2002) Nature 416, 76-81.

58. Pierson, B. K. (1994) in Early Life on Earth, Nobel Symposium, ed. Bengtson, S. (Columbia Univ. Press, New York), No. 84, pp. 161-180.

59. D’Amelio, E. D., Cohen, Y. \& Des Marais, D. J. (1987) Arch. Microbiol. 147, 213-220.

60. Joulian, C., Patel, B. K. C., Ollivier, B., Garcia, J. L. \& Roger, P. A. (2000) Int. J. Syst. Evol. Microbiol. 50, 525-528.

61. Michaelis, W., Seifert, R., Nauhaus, K., Treude, T., Thiel, V., Blumenberg, M., Knittel, K. Gieseke, A., Peterknecht, K., Pape, T., et al. (2002) Science 297, 1013-1015.

62. Grotzinger, J. P. \& Kasting, J. F. (1993) J. Geol. 101, 235-243.

63. Grotzinger, J. P. \& Knoll, A. H. (1999) Annu. Rev. Earth Planet. Sci. 27, 313-358.

64. Cloud, P. (1973) Econ. Geol. 68, 1135-1143.

65. Holland, H. D. (1973) Econ. Geol. 68, 1169-1172.

66. Cairns-Smith, A. G. (1978) Nature 276, 807-808.

67. Widdel, F., Schnell, S., Heising, S., Ehrenreich, A., Assmus, B. \& Schink, B. (1993) Nature 362, 834836.

68. Kappler, A. \& Newman, D. K. (2004) Geochim. Cosmochim. Acta 68, 1217-1226.

69. Hayes, J. M. (1994) in Early Life on Earth, ed. Bengtson, S. (Columbia Univ. Press, New York), Vol. 84, pp. 220-236.

70. Barnes, R. O. \& Goldberg, E. D. (1976) Geology 4, 297-300.

71. Boetius, A., Ravenschlag, K., Schubert, C. J., Rickert, D., Widdel, F., Gieseke, A., Amann, R., Jorgensen, B. B., Witte, U. \& Pfannkuche, O. (2000) Nature 407, 623-626.

72. Orphan, V. J., House, C. H., Hinrichs, K. U., McKeegan, K. D. \& DeLong, E. F. (2002) Proc Natl. Acad. Sci. USA 99, 7663-7668.

73. Canfield, D. E., Habicht, K. S. \& Thamdrup, B. (2000) Science 288, 658-661.

74. Shen, Y. A., Buick, R. \& Canfield, D. E. (2001) Nature 410, 77-81.

75. Huston, D. L. \& Logan, G. A. (2004) Earth Planet. Sci. Lett. 220, 41-55.

76. Drever, J. I. (1997) The Geochemistry of Natural Waters: Surface and Groundwater Environments (Prentice-Hall, Englewood Cliffs, NJ).

77. Langmuir, D. (1978) Geochim. Cosmochim. Acta 42, 547-569.

78. White, D. (2000) The Physiology and Biochemistry of Prokaryotes (Oxford Univ. Press, New York).

79. Fischer, W. W., Summons, R. E. \& Pearson, A. (2005) Geobiology 3, 33-40. 


\title{
Experimental observation of magnetosome chain collapse in magnetotactic bacteria: Sedimentological, paleomagnetic, and evolutionary implications
}

\author{
Atsuko Kobayashi ${ }^{\text {a,* }}$, Joseph L. Kirschvink ${ }^{\text {b }}$, Cody Z. Nash ${ }^{\text {b }}$, Robert E. Kopp ${ }^{\text {, }}$, \\ David A. Sauer $^{b, 1}$, L. Elizabeth Bertani ${ }^{c}$, Wim F. Voorhout ${ }^{\mathrm{d}}$, Takahisa Taguchi ${ }^{\text {a }}$ \\ a Research Institute for Cell Engineering, National Institute of Advanced Industrial Science and Technology, \\ 1-8-31 Midorigaoka, Ikeda, Osaka 563-8577, Japan \\ ${ }^{\mathrm{b}}$ Division of Geological and Planetary Sciences, California Institute of Technology 170-25, Pasadena, CA 91125, USA \\ ${ }^{\mathrm{c}}$ Division of Biology, California Institute of Technology 170-25, Pasadena, CA 91125, USA \\ d FEI Electron Optics, Applications Laboratory, P.O. Box 80066, 5600KA, Eindhoven, The Netherlands
}

Received 6 December 2005; received in revised form 16 March 2006; accepted 23 March 2006

Available online 9 May 2006

Editor: V. Courtillot

\begin{abstract}
Magnetotactic bacteria precipitate intracellular crystals of single-domain magnetite $\left(\mathrm{Fe}_{3} \mathrm{O}_{4}\right)$ and/or greigite $\left(\mathrm{Fe}_{3} \mathrm{~S}_{4}\right)$, which have often been implicated in carrying the natural remanent magnetization (NRM) of freshwater and marine sediments. In vivo, the magnetic crystals are usually aligned in chains such that their moments add together, generating net cellular moments high enough to rotate the cells passively to align with the geomagnetic field. A magnetostatic/biophysical analysis demonstrates that this arrangement is out of dynamic equilibrium and would collapse spontaneously without a support mechanism. Past rock magnetic analyses of shallow water marine carbonates suggest that partial collapse does occur during diagenesis and dolomitization.

To calibrate this effect we induced magnetosome chain collapse in Magnetospirillum magnetotacticum strain MS-1 by progressive sonification and treatment with detergents and monitored the changes with rock magnetic analysis and TEM. Although it has been speculated that the cell wall and associated membrane structures act to prevent magnetosome chain collapse, our data indicate that magnetosome linearity persists long after cells are disrupted. This is consistent with prior observations that in some magnetotcocci the magnetosome chains pass through the cell interior, precluding continuous contact with the cell wall and implying additional support structures exist in some species.

Using TEM tomographic reconstructions prepared with a magnetic technique that prevents chain collapse, we examined the three dimensional ultrastructure of magnetosomes without the problem of post-mortem magnetosome motion. This method revealed the presence of an intracellular organic sheath beyond that of actin-like filaments reported recently that follows the chain of magnetosomes, which we postulate evolved to hold the crystals in place and enhances their ability to preserve NRM in sediments. As the genomes of two magnetotactic bacteria contain several apparent homologues of known eukaryotic cytoskeletal proteins, natural selection for magnetotaxis may have played a role in the evolution of precursors to the eukaryotic cytoskeleton.
\end{abstract}

\footnotetext{
* Corresponding author. Tel.: +81 72751 9524; fax: +81668440595.

E-mail address: kobayashi-a@aist.go.jp (A. Kobayashi).

${ }^{1}$ Present address: Department of Pathology, Oregon Health and Science University, Portland, OR 97202, USA.
} 
The presence of this sheath is also consistent with the observation of electron translucent material associated with putative magnetofossil chains in ALH84001.

(C) 2006 Elsevier B.V. All rights reserved.

Keywords: rock magnetism; paleomagnetism; magnetotactic bacteria; cytoskeleton; evolution

\section{Introduction}

In living magnetotactic bacteria, individual magnetite crystals are formed within a string of vesicles, each of which is a lipid-bilayer membrane [1-3]. Numerous optical and behavioral observations show that the in vivo magnetite crystals are aligned in linear chains, allowing virtually all of their dipole magnetic moments to sum linearly, bringing the total magnetic moment per cell to values close to that expected from the quantity of single domain magnetite or greigite within them [3-9]. Natural selection for magnetotaxis has presumably optimized the use of iron within many of these cells, as it is a scarce nutrient that, in aerobic environments, requires an energy-expensive active transport system [10]. However, the ability to keep the magnetosomes aligned is a biophysical puzzle because lipid-bilayer membranes are fluidic, and the magnetite crystals ought to be able to move and rotate freely within their vesicles. Similarly, magnetite-containing vesicles should be able to move relative to each other in response to local magnetostatic interactions unless otherwise constrained [11].

One model suggests that the apparent stability of magnetosome chains may be due to a combination of the magnetosome shape and elastic properties of biological membranes $[12,13]$. This model is based on 2D TEM images of in situ magnetosomes that suggested the crystal shapes were simple right cylinders or rectangular prisms with flat ends. However, subsequent HRTEM studies of magnetosomes have revealed that the ends are, in fact, not square - they are typically 'rounded' by the expression of $\{100\},\{110\},\{111\}$ and possibly other crystal faces $[14,15]$. This geometry acts to minimize the stabilizing effect of the flat ends and implies that magnetosome chains are intrinsically unstable - as the rounded ends of two adjacent crystals start to rotate out of linear alignment, there is no barrier to inhibit chain collapse. Thermal fluctuations in the membrane surface also appear large enough to initiate chain collapse [16]. This rounding at magnetosome ends has been explained as a mechanism for improving the saturation magnetization, and hence the biological efficiency of iron utilization, by eliminating spin-vortex 'flower-structures' that arise near sharp corners of single-domain crystals $[17,18]$.
Although numerous TEM images show rapidly fixed magnetotactic bacteria with apparently linear chains, Vali and Kirschvink [19] note that this is not always the case in many bacteria. Despite the evidence for linear chains in vivo, chains do collapse during typical preparation for TEM; this has been independently predicted and observed by Philipse and Maas [20]. In particular, even apparently linear images of such chains, when viewed via a single 2D electron shadow image in a TEM, cannot detect oscillations in the vertical plane. Linear chains can in principle provide a strong and stable natural remanent magnetization (NRM), whereas highly interacting clumps of SD particles would have both lower intensity and lower stability. The ability to avoid clumping is critically important for understanding how magnetofossil-rich sediments are able to preserve NRM.

Using rock magnetic techniques, McNeill and Kirschvink [21] have documented magnetofossil chain collapse in carbonaceous marine sediments from the Bahamas, where the process reduces the intensity and stability of the NRM. In particular, increasing levels of diagenesis, calcification, and dolomitization inhibited the acquisition of anhysteretic remanent magnetization (ARM), implying that the magnetostatic interaction between magnetic particles was increasing [22]. However, it was not clear from that study how the microscopic arrangements of the magnetofossils were changing, as the procedures for extracting the bacterial magnetite particles (magnetofossils) from sediments result in clumped aggregates of particles.

Without some form of rigid support, linear chains of single-domain magnetite crystals will collapse spontaneously because they are physically unstable. For elongate crystals, magnetostatic energy calculations $[10,20]$ show that the collapse of chains into clumps of crystals is favored strongly, as, by reducing the center-to-center spacing of the dipoles, it can reduce the magnetostatic potential energy of the cell by $\sim 10^{4} k T$ (where $k T$, the product of the Boltzmann constant and the absolute temperature, is the one-dimensional thermal background energy). Paired anti-parallel magnetosome crystals have been seen in TEM images of cells with elongate crystal morphologies (e.g., [19]). Although equant crystals are more stable in a chain configuration than in the paired anti-parallel configuration, for any 
crystal shape the overall magnetostatic energy of long chains of particles is minimized when the chains collapse into small loops.

At least two mechanisms could prevent the collapse of these chains. First, proteins or some other organic macromolecules could hold adjacent crystals together like strings by anchoring themselves into the internal structure of each crystal. These anchor strings would have to pass completely through two lipid bilayer membranes, be of the correct length to minimize inchain wobble, and be strong or numerous enough to overcome forces producing chain collapse. The ends of the chain would then be attached to the structural components of the cell wall, to transmit the rotational magnetic torque to the entire cell body [5]. The other possibility is that the cells might produce a larger organic 'sheath' that would enclose the entire chain of magnetosomes within a rigid superstructure, preventing their collapse $[23,24]$. If held in a line, individual crystals would be inhibited from rotating within their encapsulating membrane by magnetic interactions with adjacent particles, rather than with embedded strings. This sheath structure could then be anchored to various cytoskeletal filaments that run along the inside of the bacterial cell membranes to provide cell shape and rigidity $[25,26]$, thereby transferring the magnetic torque needed for navigation to the entire cell. A protein in the magnetosome membrane, MamJ, has been shown to be necessary for alignment of magnetosomes along a filamentous structure in the magnetotactic bacteria Magnetospirillum gryphiswaldense [27], and in Magnetospirillum magneticum filaments of an actin-like protein, MamK, appear to run the length of the cell [28], supporting the idea that external structures constrain the motion of the magnetosomes

To distinguish between these possibilities, we first performed a series of experiments with cultures of $M$. magnetotacticum strain MS-1, which we subjected to increasing cellular disruption using ultrasonic and chemical methods; TEM imaging and rock magnetic experiments were coupled to monitor the progress of chain collapse. Next, we developed a simple method to inhibit the magnetostatic motion of the chains during preparation for TEM, from which we can then derive accurate parameters such as magnetosome volume and spacing using energy-filtered TEM. These data are then used to estimate the energies and forces involved in the stability of magnetosome chains. Next, we report on the tomographic 3D reconstructed imaging of cells prepared in this fashion. Finally, we report results of our search for supporting structures along the magnetosome chains.

\section{Materials and methods}

\subsection{Materials}

Cultures of the magnetotactic bacterium M. magnetotacticum strain MS-1 were obtained from the American Type Culture Collection (ATCC 31632), and grown according to their recommendations. When cultures had reached their log-growth phase, magnetotactic cells were harvested from aggregations accumulating next to magnets held at the side of the culture flasks using Pasteur pipettes modified to have a bent tip, as done by Moench and Konetzka [29].

\subsection{Disaggregation method}

About $30 \mu \mathrm{l}$ aliquots of cell pellets were placed in acid-washed plastic Eppendorf tubes, and diluted in an equal volume of deionized water. Disruption began by inserting the stainless-steel probe of a Fischer Scientific Sonic Dismembrator $550^{\circledR}$ into the liquid, at increasing levels of exposure/pulse cycles. To further disrupt the cells by disrupting the membranes, we added steps including sodium dodecyl sulfate (SDS). In order of progressive disruption, our samples reported here were treated in the following sequence: (a) wild-type cells with no disruption, (b) disruption with 200 pulses at $8 \mathrm{~s}$ each, (c) 400 pulses at $7 \mathrm{~s}$ each, (d) $0.05 \%$ SDS, and 75 pulses at $5 \mathrm{~s}$ each, and (d) $0.05 \% \mathrm{SDS}$, and pulsed every $4 \mathrm{~s}$ for $10 \mathrm{~min}$. The pellets frozen in the Eppendorf tubes were subjected to a standard series of rock magnetic analyses as noted below.

\subsection{Magnetometry measurements}

These rock magnetic experiments were done at $-20{ }^{\circ} \mathrm{C}$, using a vertically mounted $2 \mathrm{G}$ Enterprises ${ }^{\circledR}$ SQUID magnetometer housed in a magnetically shielded clean room at Caltech, which was equipped with a refrigerated sample access chamber. A stepping motor raised and lowered the sample from above on thin monofilament line, moving it between the coils and the SQUID sense region. Experiments involved the following techniques: (1) Alternating-field (AF) demagnetization up to $100 \mathrm{mT}$ was done with a commercial degausser (Applied Physics Systems ${ }^{\circledR}$ of Menlo Park, CA), using a solenoid mounted at the top of the sample path. (2) To supply DC biasing fields between 0 and $2 \mathrm{mT}$, a custombuilt Lee-Whiting-4 coil system [30,31] was mounted over the Af solenoid, but within the $\mu$-metal shielding. (3) Isothermal remanent magnetizations (IRMs) were applied using a capacitive impulse magnetizer (ASC 
Scientific ${ }^{\circledR}$, Model IM-10, with the design of Kirschvink [32]), which discharged through a solenoid mounted along the sample path above the AF coil. All of these devices were run automatically by a dedicated computer that had been programmed to do, in sequence, the following progressive magnetization and demagnetization experiments: (1) ARM acquisition in a $100 \mathrm{mT}$ AF field, with biasing fields up to $2 \mathrm{mT}$; (2) AF of the maximum ARM up to $100 \mathrm{mT}$; (3) IRM acquisition up to $100 \mathrm{mT}$; and (4) AF of the IRM. These data constitute the basic magnetic granulometry techniques of Cisowski [22] and the ARM modification of the Lowrie-Fuller test [33]. Control measurements were done with similar acid-washed Eppendorf tubes filled with sterile growth media to ensure that the magnetic signals were due to the bacteria and not to the contaminants from the culture media or disposable labware [34].

\subsection{Transmission electron microscopy}

For the disaggregation experiments, we preserved the disrupted configurations for both TEM and rock magnetic experiments by rapidly blotting small drops of the cell suspension onto hydrophilic TEM grids, while at the same time freezing the remaining cell suspension rapidly in liquid nitrogen for rock magnetic analyses. The grids were examined at moderate magnification on a Zeiss-9 TEM to assess chain linearity vs. clumping. For the experiments with aligned chains, the samples were prepared by negative staining or conventional embedding procedures (e.g., [35]) as described elsewhere. We stabilized magnetotactic bacteria with a strong background magnetic field maintained during the entire fixation and TEM sample preparation process to prevent chain collapse with a pair of $\mathrm{NdFeB}$ disc magnets, $2 \mathrm{~mm}$ in diameter separated by a $3.7 \mathrm{~mm}$ gap, which produced a measured minimum magnetic field in the center of $\sim 100 \mathrm{mT}$ (see Fig. 3 for details). The magnetostatic alignment of the chains also facilitates TEM-based ultrastructural analysis, as ultrathin sections can be made from embedded samples with a diamond knife cutting parallel to the chain length.

Samples were prepared by negative staining or conventional embedding procedures (e.g., [35]). All cells collected by magnets were first fixed with $1 \%$ glutaraldehyde and $4 \%$ paraformaldehyde in $0.1 \mathrm{M}$ phosphate buffer (PB) at $\mathrm{pH} 7.4$ for half an hour. Cells were then post-fixed in $1 \%$ osmic acid/0.1 M PB for $1 \mathrm{~h}$ and run through the standard epon embedding procedure. Samples were cut into $200 \mathrm{~nm}$ thin sections and $70 \mathrm{~nm}$ ultrathin sections parallel to the chain length by a Leica Ultracut UCT with a diamond knife. Each specimen was collected on $\mathrm{Cu}$ grid supported by carbon films, then post-stained with $2 \%$ uranyl acetate/lead citrate. A few cells were negatively stained with tungsten phosphate as noted below.

We used a Tecnai F20G2Twin, equipped with Gatan energy filter GIF2002 and multi-scan CCD camera (1024 $\times 1024,24 \mu \mathrm{m} / \mathrm{pixel})$, comprising a phosphor fiber-optically coupled, operating at $100 \mathrm{kV}$. The filter was tuned for the chromatic-geometric aberrations, prior to image acquisitions at the magnification. The images of iron were acquired with an energy filter slit width of $25 \mathrm{eV}$ for mapping the Fe-L edge at $708 \mathrm{eV}$. We obtained images with acquisition times of $30 \mathrm{~s}$; our instrument sample holder has an rms maximum drift of $\sim 0.5 \mathrm{~nm} / \mathrm{min}$. We corrected for baseline change using the standard 3-window correction method that produces an optimum isolation of the Fe-signal from the background. All images were corrected for the CCD's dark current and gain variations and processed by Digital Micrograph 3.3 for size determinations.

\subsection{Electron tomographic imaging}

In the magnetotactic bacteria, structures supporting the magnetosome chains should be visible with suitable TEM techniques, assuming that the spatial relationship between the crystal chains and the supporting materials can be preserved post-mortem. Automated electron microscopic tomographic imaging $[36,37]$ can then provide another tool for examining the continuity between the magnetosome chains and internal cellular structures, particularly when the structure has a directional irregularity. A series of projection images is acquired by tilting the specimen incrementally to high tilt angles around an axis perpendicular to the electron beam. From this series of images, a 3D tomogram with nanometer-scale resolution can be reconstructed. For our tomographic study, we used a Tecnai Sphera with a sample holder, tilting at $1^{\circ}$ increments through $\pm 65^{\circ}$, operating at $200 \mathrm{kV}$. For a $200 \mathrm{~nm}$ spherical sample incrementally tilted up to $\pm 65^{\circ}$ with $1^{\circ}$ steps, the limiting resolution is around $5 \mathrm{~nm}$ [38]. The drift of the holder position was calibrated prior to the data acquisition for experimental samples. Image data were processed using Xplore3D software fort 3D reconstruction and further processes were done with the AMIRA 3.0 software package.

\section{Results}

\subsection{Dispersion of aligned chains}

Figs. 1 and 2 show representative TEM and rock magnetic results from the dispersion experiments. As 


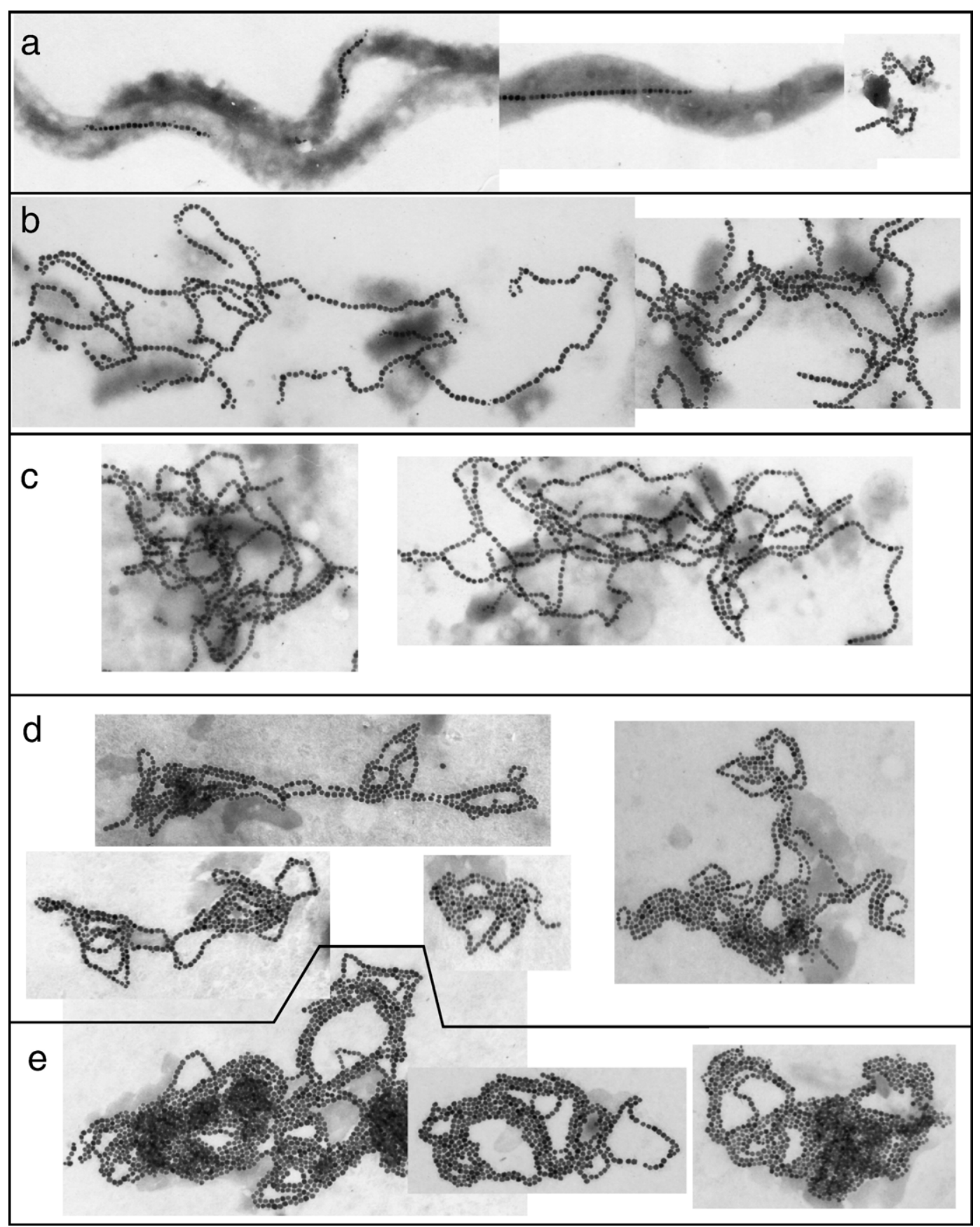

Fig. 1. TEM images of the progressive collapse of the magnetosome chain structures in cells of M. magnetotacticum strain MS-1. The order of progressive disruption is arranged vertically such that $(\mathrm{a})<(\mathrm{b})<(\mathrm{c})<(\mathrm{d})<(\mathrm{e})$. Note that both the outer and plasma lipid-bilayer membranes surrounding the cell are lost at the first ultrasonic disruption step used in the (b) preparation; although the magnetosome chains start to bend, they do not collapse. For scale, note that magnetosomes in this strain have a center-to-center separation of approximately $57 \mathrm{~nm}$.

the cells or cell fragments were observed optically to adhere tightly to the hydrophilic surface of the TEM grids before air drying, and the freezing was rapid, it is reasonable to assume that the transfer and freezing procedures had minimal effect on the magnetosome organization, other than compressing 3D loops and clusters onto a 2D TEM grid. Undisrupted cells are shown in Fig. 1a, and the red curves labeled (a) in Fig. 2. The TEM images reveal that progressively increasing levels of ultrasonic disruption and detergent 
a

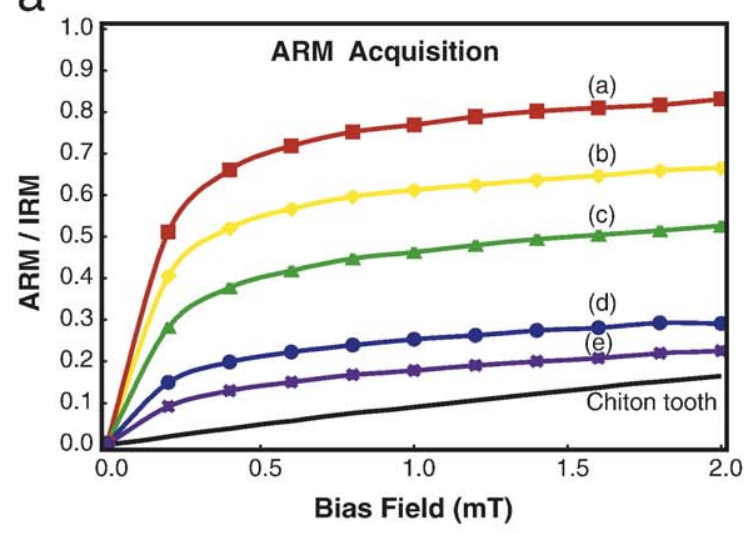

b
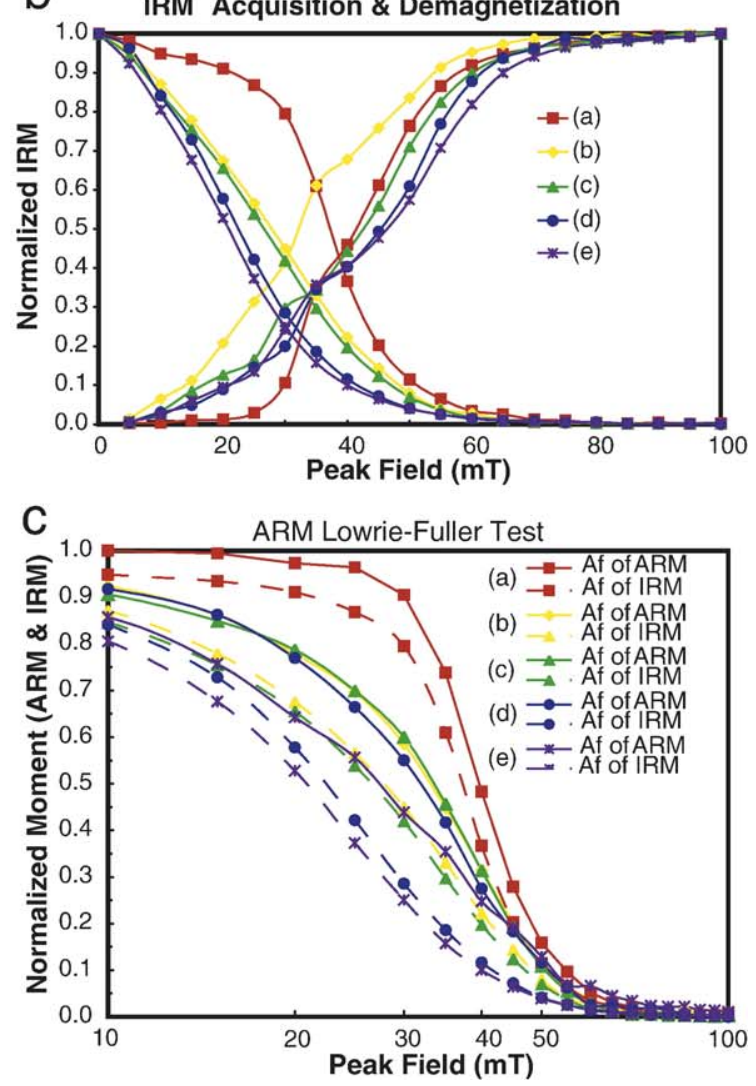

Fig. 2. Rock-magnetic experiments on aliquots of the cells imaged in Fig. 1, with color patterns in rainbow order of increasing disruption. (a) Acquisition of anhysteretic remanent magnetization, (b) LowrieFuller ARM test, (c) comparison of coercivity gain/loss between IRM and ARM.

destruction of the membranes led to an increasing frequency of closed flux-loops and dense clumps. Although the first ultrasonic disruption (b) destroyed most visible cell structures (presumably by popping both the lipid-bilayer membranes which form the cell wall/envelope in Gram-negative bacteria), the chains themselves did not collapse immediately into disordered clumps. Instead, they remain associated with fragments of the cytoplasm until that could be removed by the action of detergents and higher levels of ultrasonic treatment.

Rock magnetic data display a trend of increasing interaction effects that increase with cell disruption, including a lowering of the ARM acquisition rate (Fig. 2a) and a rather abrupt decrease of the median coercivity (Fig. 2b, c). This loss of coercivity is intriguing, because the rather sharp drop-off of the IRM-AF curve between 35 and $45 \mathrm{mT}$ (Fig. $2 \mathrm{~b}$ and c) happens before the intersection point of the acquisition/demagnetization drops (the interaction " $R$ " value of Cisowski [22]). All of the samples pass the Lowrie-Fuller ARM test for single-domain behavior (Fig. 2c): the ARM-AF curves lie above those of the IRM-AF.

\subsection{Electron microscopy}

As shown in Figs. 3-5, the strong-field embedding technique provides samples with remarkably straight and uniformly parallel magnetosome chains, maximizing the ability to observe cellular material in their vicinity. Close examination of the TEM images prepared in this fashion indicates that the magnetite crystal chains in MS-1 are associated with an electron translucent sheath; this is indicated on the $50-70 \mathrm{~nm}$ thick wedge of Fig. 3a and b. Fig. 3c shows results from an electron microscope image of a negatively stained, fixed MS-1 whole cell prepared in this fashion; Fig. $3 \mathrm{~d}$ is a higher resolution image of one end of this chain. Small red arrows in Fig. $3 \mathrm{~b}$ and $\mathrm{d}$ show the somewhat irregular margins of this apparent sheath material. Detailed measurements indicate that the margins of this sheath are somewhat irregular, but that the sheaths are approximately $100 \mathrm{~nm}$ in diameter. This implies that it is not simply an extension of the lipid-bilayer magnetosome membrane that covers each magnetite crystal $[1,2]$, which should only extend $\sim 6 \mathrm{~nm}$ from the crystal surfaces, but that it is composed of additional material. Two movies of the tomographic reconstructions are available from the online supplemental information, and stereo pairs of images extracted from these are shown in Fig. 4. An additional tomographic reconstructed image in the Appendix shows the high degree of internal control on the magnetosome spacing and positioning in the bacterial cell.

To estimate the fraction of the cellular volume occupied by the magnetosome chain assembly, we modeled the magnetosomes of Figs. 3 and 5 as regular 

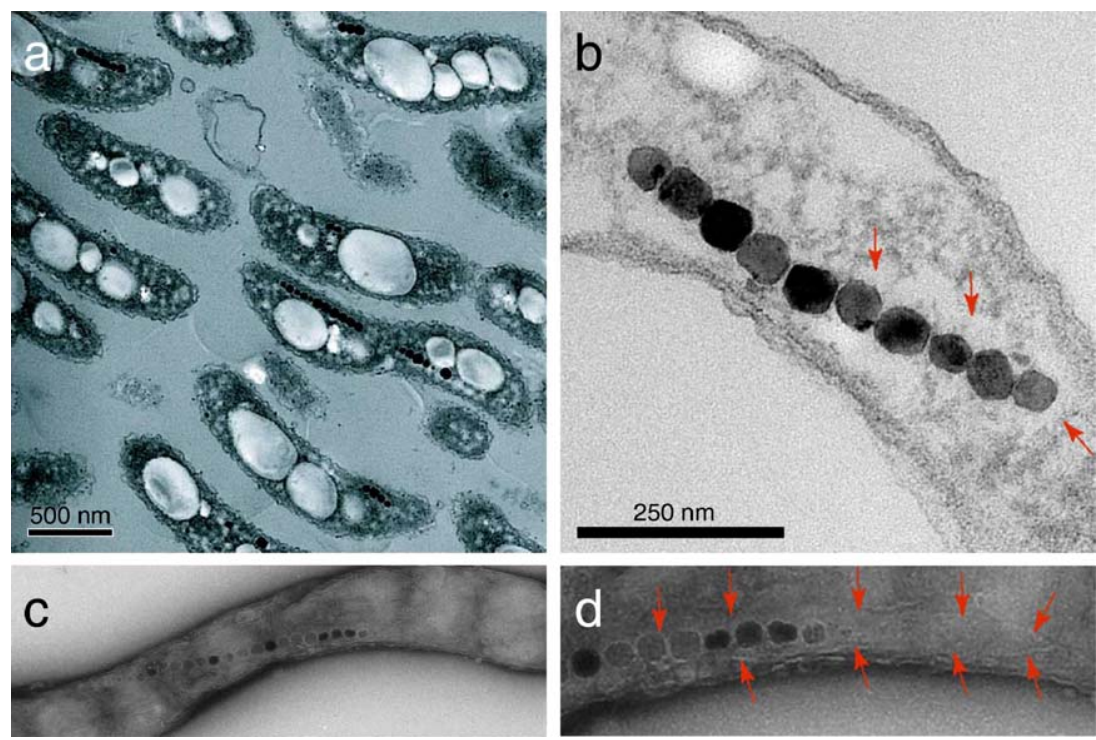

Fig. 3. TEM images of intact cells of MS-1, prepared in the presence of a static, strong magnetic field designed to prevent magnetosome chain collapse. The magnetostatic orientation energy ( $E_{\text {mo }}$, in cgs units) of each magnetosome in an external magnetic field, $B$, is given by their vector dot product, $-\mu \cdot B$, where $\mu$ is the particle magnetic moment. (For simplicity in magnetostatic calculations, we prefer to use the Gaussian cgs system and translate our results back into SI units.) If this exceeds the collapse energy $\left(E_{\mathrm{c}}\right)$ between two particles separated by a distance $x$, given by $-\mu^{2} / x^{3}$ (see Section 3.3 regarding the biophysics), the chain structure is stabilized; for magnetosomes of $M$. magnetotacticum, strain MS-1, this critical threshold field is $\sim 16 \mathrm{mT}$. (a and b) $\sim 70 \mathrm{~nm}$ thick ultra-thin section of wedge of magnetotactic bacteria embedded in epon, cut parallel to the direction of the applied magnetic field direction. (c) Negatively stained TEM image of an intact bacterial cell, showing the linear chain of electron dense magnetosomes. (d) Enlarged view of the right-hand margin of the magnetosome chain in (c). Red arrow tips in (b) and (d) show the margin of the organic 'sheath' that envelopes the magnetosome chain structures. Only the cells in (c) and (d) were negatively stained with tungsten-phosphate (Nano-W, Nanoprobes).

truncated octahedra ('cubo-octahedra' using the terminology of [39]), and the sheath as a simple cylinder surrounding the magnetosome chain. In the magnetospirilla, numerous studies have shown that the crystals tend to be aligned with their crystal axes parallel to the [111] cubic direction (i.e., they have parallel sets of $\{111\}$ crystal faces perpendicular to the chain axis). This is not surprising because the [111] axis is the magnetically 'easy' direction, and crystallites growing in the strong local field at the end of a magnetosome chain will spontaneously align in this orientation [11]. As the plane of our TEM sections shown in Figs. 3 and 5 were cut parallel to the direction of the strong field applied by the rare-earth magnets, the crystal dimension measured along the chain axis provides an estimate of the spacing between parallel $\{111\}$ planes. Since the energy loss at the $\mathrm{Fe}-$ $\mathrm{L}$ edge at $708 \mathrm{eV}$ is very sharp and our samples have a large signal/noise ratio, we measured the crystal size and spacing by locating the full width half-maximum (FWHM) intensity point in the Fe spectra as shown on the line profile in our Fig. 5.

Measurements of 15 mature crystals from two magnetosome chains like those shown in Fig. 4 yield a mean plane separation of $43.1 \pm 4.5 \mathrm{~nm}$, with a centerto-center spacing of $56.8 \pm 3.7 \mathrm{~nm}$ ( $1 \sigma$ errors); the average interparticle gap of $\sim 13.7 \pm 2.1 \mathrm{~nm}$ is consistent with two unit membranes. This size distribution is in good agreement with the measurements of Devouard et al. [39], who also did not use negative staining but had no $3 \mathrm{D}$ control of the chain warping. Our measured interparticle spacing, however, is significantly larger than the $10.0 \mathrm{~nm}(2 \times 5.0 \mathrm{~nm})$ spacing reported by Gorby et al. [1], which may be a result of the negative staining procedure enhancing the apparent size of the crystals. (Although small, this is an important difference because the magnetostatic forces vary as the inverse fourth power of particle separation.)

To convert from this on-axis $\{111\}$ planar separation to an estimate of the cubo-octahedral crystal volume involves using geometry; the length of any of the twelve edges of a regular octahedron is $\sqrt{ } 6$ times that of the distance from the center to the midpoint of any of the faces. Using a right triangle, the $43 \mathrm{~nm}$ average planar spacing corresponds to a regular octahedron with a full vertex-to-vertex edge length of $53 \mathrm{~nm}$. Although the M. magnetotacticum crystals are somewhat variable, the six octahedral vertices are 

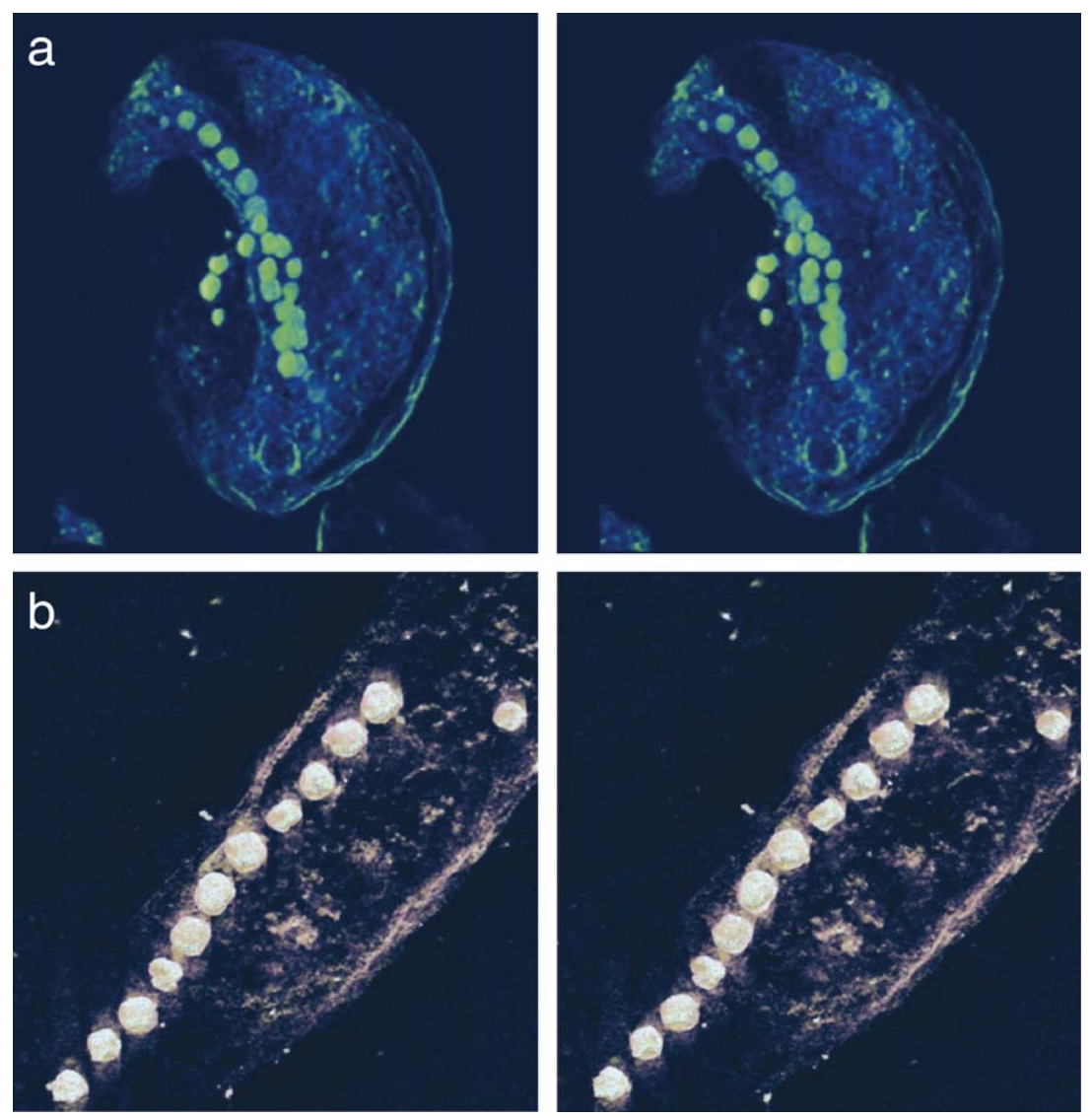

Fig. 4. Stereo frames extracted from tomographic reconstructions of two samples of M. magnetotacticum strain MS-1, using $11^{\circ}$ tilt between frames. Samples for (a) and (b) are cells from fresh cultures grown with the same techniques, but embedded in the absence and presence, respectively, of a strong magnetic field for keeping the magnetosome chains from collapsing, as mentioned in the text. The specimens were prepared by $200 \mathrm{~nm}$ thick sectioning. These correspond to the supplementary data in the Appendix and are available at http://www.gps.caltech.edu/ nascz/Movie_A.mov and http://www.gps.caltech.edu/ nascz/Movie_B.mov.

truncated by $\{100\}$ and equivalent planes, removing about a third of each edge length at each vertex. This truncation reduces the volume of a perfect octahedron by that of 6 square-based prisms located at each vertex, giving a best volume estimate per crystal of about $62,500 \mathrm{~nm}^{3}$. For comparison, the diameter of a sphere with an equal volume is about $49 \mathrm{~nm}$. The volume of each magnetosome (magnetite plus membrane) is found in a similar fashion to be about $142,500 \mathrm{~nm}^{3}$, a bit more than double that of the magnetite alone. Using $\sim 100 \mathrm{~nm}$ diameter for the observed sheath, with the length of $\sim 2000 \mathrm{~nm}$ seen in Fig. 3a, implies that the entire magnetosome and sheath assembly has a cylindrical volume of $\sim 15.7$ million $\mathrm{nm}^{3}$, which is about $3 \%$ of total volume of the cell. This is a significantly larger estimate for the cellular volume devoted to magnetotaxis than previous estimates of about $0.5 \%$, which only considered the magnetosomes [6].

\subsection{Biophysics}

In vector notation the magnetostatic energy for a pair of interacting magnetic dipoles, $\boldsymbol{\mu}_{1}$ and $\boldsymbol{\mu}_{2}$, separated center-to-center by a displacement vector, $\boldsymbol{x}$, is given by:

$\mathrm{E}_{1,2}=\frac{\overrightarrow{\boldsymbol{\mu}_{1}} \cdot \overrightarrow{\boldsymbol{\mu}_{2}}-3\left(\overrightarrow{\boldsymbol{\mu}}_{1} \cdot \overrightarrow{\boldsymbol{n}}\right)\left(\overrightarrow{\boldsymbol{\mu}_{2}} \cdot \overrightarrow{\boldsymbol{n}}\right)}{|\boldsymbol{x}|^{3}}$

where $\boldsymbol{n}$ is a dimensionless unit direction vector parallel to $\boldsymbol{x}$ [40]. For a pair of equal magnetic dipoles aligned head-to-tail in parallel, the magnetostatic interaction energy $\left(E_{\text {parallel }}\right)$ reduces to $E=-2 \boldsymbol{\mu}^{2} / \boldsymbol{x}^{3}$. Similarly, the force $(F)$ between the particles is the spatial gradient of the energy, $F=6 \boldsymbol{\mu}^{2} / \boldsymbol{x}^{4}$. These values are halved for particles that are clumped with their moments in a sideto-side antiparallel configuration; when $\boldsymbol{\mu}_{1}$ and $\boldsymbol{\mu}_{2}$ are antiparallel, and both are perpendicular to $\boldsymbol{n}, E=-\boldsymbol{\mu}^{2} / \boldsymbol{x}^{3}$ and $F=3 \boldsymbol{\mu}^{2} / \boldsymbol{x}^{4}$. Hence, for two interacting uniformly 


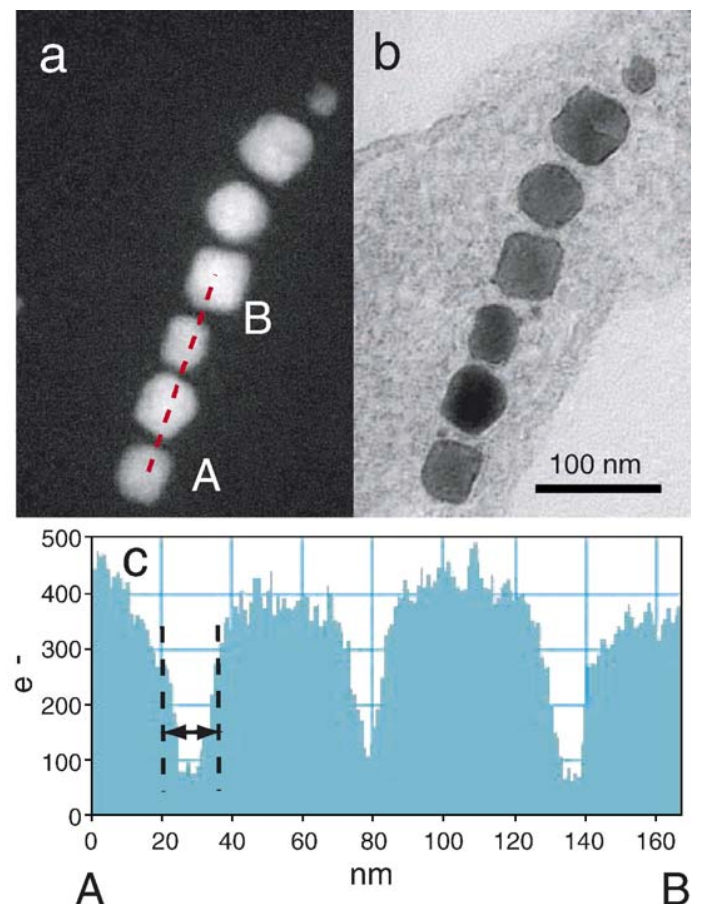

Fig. 5. Image of intact cells of MS-1 for size measurement. (a) Energyfiltered image of iron. (b). Zero loss image of (a). (c) Intensity profile of (a), taken between points $\mathrm{A}$ and $\mathrm{B}$ in (a). The crystal size is determined by the distance between two edge points at full width halfmaximum (FWHM).

magnetized spheres the minimum energy configuration is a head-to-tail configuration. However, for elongate chains of spheres in a negligible background field (e.g., [41]), the magnetostatic energy stored in the field itself becomes large, and this can be reduced by curling the chains into arcs or loops, as clearly happened in the bacterium shown in Fig. 4 a.

As noted above, magnetite crystals produced by Magnetospirillum strain MS-1 are equivalent to spheres of $\sim 57 \mathrm{~nm}$ diameter, and have about $13.7 \mathrm{~nm}$ of organic material holding them apart. High-purity, low-defect magnetite such as that precipitated by the magnetotactic bacteria has a saturation magnetization of $4.8 \times 10^{5} \mathrm{~A} / \mathrm{m}$ (480 emu $\left./ \mathrm{cm}^{3},[42]\right)$. This yields magnetic moments of the particles of $\sim 4.3 \times 10^{-17} \mathrm{~A} / \mathrm{m}^{2}$, and an interparticle compressional force at this separation of about $120 \mathrm{pN}$. The head-to-tail binding energy is about $-550 \mathrm{kT}$, implying that the arrangement is quite stable against thermal disruption.

\section{Discussion}

In biomagnetic and rock magnetic studies, the in situ packing geometry of single domain grains is an important, but commonly difficult, parameter to determine. The calibrations shown in Figs. 1 and 2 provide a qualitative guide to how these interaction effects vary as a function of the packing geometry, and provide a start at how to interpret data from natural samples like Recent muds from the Bahamas $[21,43]$. Of particular interest is the immediate broadening of the coercivity spectra with the initiation of chain curling (the (a) to (b) steps of Figs. 1 and 2). This is accompanied by an abrupt decrease in median coercive field from about 40 to $30 \mathrm{mT}$, but without a significant drop in the $R$ value (the intersection point of the IRM and AF of IRM curves in Fig. 2b, a measure of interparticle interaction [22]). The drop in coercivity is presumably due to the disruption of the linear chain structure - bends in the chains will reduce the stabilizing influence of adjacent magnetic moments to each particle, making it easier for their moments to flip. Upon further disaggregation (the (b) to (c) steps of Figs. 1 and 2), the TEM images indicate that the magnetosomes start to form more regions where the chains line up next to each other in parallel strings; this is accompanied by a slight increase in median coercivity (from about 30 to $35 \mathrm{mT}$ ), but with a decrease in the $R$ value from 0.42 to 0.32 in Fig. 2 b. We suspect that this slight increase in coercivity may be due to the effect of single-domain grains in pairs of adjacent chains stabilizing each other against the external magnetic field (AF or pulse) that is attempting to change their magnetic direction. The drop in $R$ value is an indication of the increase in interparticle interaction [22]. Further disruptions including treatment with SDS (the (d) and (e) steps of Figs. 1 and 2) start to destroy the chains, and result in dense 3D clusters of strongly interacting particles. These yield rock magnetic results that progressively resemble the end member of the chiton tooth (Cryptochiton stelleri) standard $[22,44]$.

We were surprised at the difficulty of disrupting magnetosome chains in lab cultures. Our initial hope was to disrupt them so completely with the powerful ultrasonic treatment that individual magnetite crystals would separate from each other and become magnetically isolated in the medium, which would allow us to distinguish the magnetic properties of the individual crystals from the properties that arise due to the chain organization. Were this to happen, we would expect that the ARM acquisition curve shown in Fig. 2a would steepen. Instead, progressive disruption from the chain structures dramatically increased the interaction effects, eventually producing the clumped aggregates and loops shown in Fig. 1e. 
Comparing these data with the rock-magnetic study of Recent sediments of the Bahama banks [21] indicates that the majority of the Bahama samples have not reached total chain collapse. In this configuration, the particle clumps will still have a net magnetic moment that can align in the geomagnetic field, and, as the sediments are lithified by the precipitation of carbonate cements, this will preserve a stable but weaker NRM, as is often observed in shallow-water carbonates [43]. We also note that post-depositional growth of organisms like Geobacter that precipitate extracellular magnetite (e.g., [45]) might also produce this change in the ARM properties of sediments with depth; however, this would lead to an increase in the IRM intensity which is not observed [21].

An important observation here is the fact that the initial disruption of the periplasmic and cytoplasmic membranes did not lead to total collapse of the magnetosome chains. At present, we do not know how these are held together within the cell, although it is often suggested that chain stabilization in the magnetotactic bacteria is produced by continuous physical links between the chain structure and the hydrostatically supported cell walls $[1,3,6,46]$; this has apparently been observed recently in M. magneticum strain AMB-1 [28]. However, if these connections to the membrane were the only method of support, the chains should collapse immediately once the membranes are disrupted. The fact that this collapse requires much more severe treatment, including chemical denaturation agents, demands the presence of additional cytoplasmic and ultrastructural supporting structures that are resistant to disaggregation.

Recent work has demonstrated the presence of filamentous structures along which magnetosomes are aligned in the closely related species M. gryphiswaldense MSR-1 [27] and M. magneticum AMB-1 [28]. Using cryo-electron tomography, both groups observed filaments that spanned the length of the cell, identified as most likely as the actin homolog, mamK [28], and apparently held in place by the protein product of gene mamJ [27]. In wild-type cells the magnetosomes were aligned along this filament, while in MamJ-deficient mutants the magnetosomes were not associated with the filament, instead forming clumps. With their technique, no sheath structure was observed even in the wild-type. While AMB-1, MSR-1, and MS-1 are closely related, they do have different populations of magnetosome membrane proteins [47], such that they may have different support structures for their magnetosome chains. An intriguing possibility, however, is that MamJ is required for sheath formation, or is one of many components of the sheath itself.
Further evidence for the existence of such supporting structures comes from past freeze-fracture replica studies of magnetic cocci which reveal the presence of linear magnetosome chains that are structurally distant from the cell wall [19]. As shown in Fig. 6a (Fig. 3c of Vali and Kirschvink [19]), these cocci contain large, spherical intracellular storage granules up to $1 \mu \mathrm{m}$ in size. The freeze-fracture plane in Fig. 6a not only transected the entire cell, exposing a chain of magnetosomes, it also cut through one of these storage granules near its maximum diameter. Hence, the freeze-fracture plane went through the center of the cell, not simply tangential to the edge. As shown schematically in Fig. $6 \mathrm{~b}$, the storage sphere contacts the cell membrane on one side, and the magnetosome chain on its other side. In a spherical cell the center does not come near the
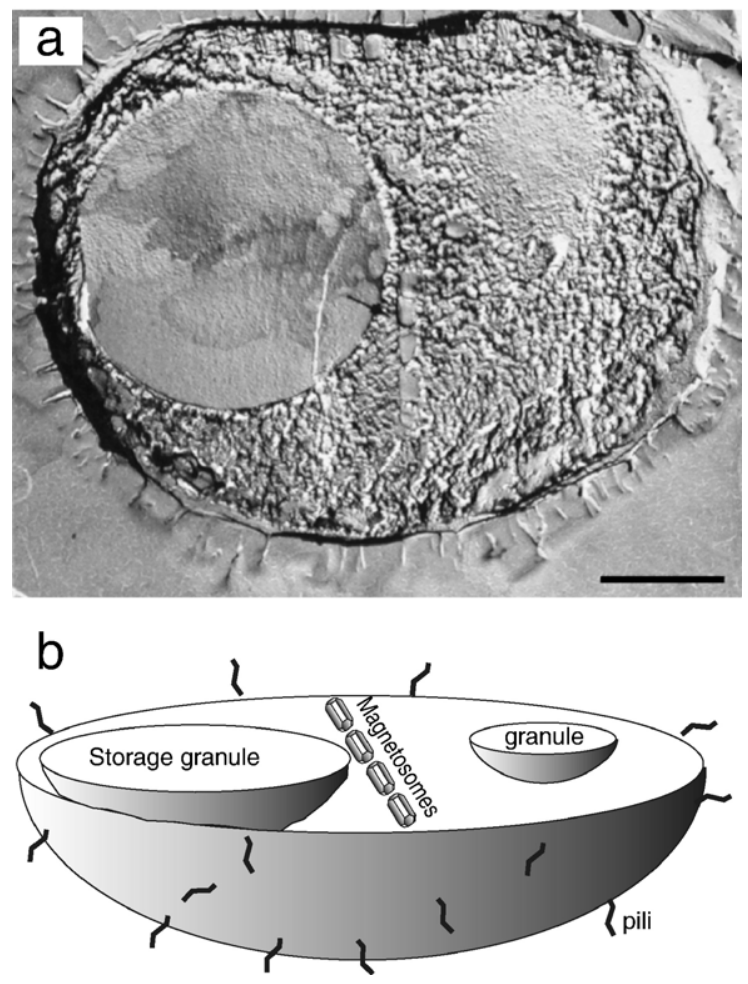

Fig. 6. (a) Magnetic coccus from a freshwater pond near the town of Landshut in Germany (from Vali and Kirschvink [19], reproduced with permission). This is a freeze-etched replica from a transverse section through the center of one of these cells; the large spherical structure is a phosphate granule that has been transacted near its full dimension, indicating that the fracture plane penetrated near the center of the nearly spherical cell. The small arrow indicates the location of the magnetosome chains, which is located in the interior of the cell away from the wall and therefore lacking support. The scale bar is $0.5 \mu \mathrm{m}$ in length. (b) Schematic diagram of the freeze-fracture image of (a), showing the location of the intracellular storage granules and the magnetosome chain. The central portion of the chain is not adjacent to the cell membrane, but goes through the center of the cell. 
bubble-like membrane, so the magnetosome chain shown in Fig. 6a cannot be in contact with it, except possibly at the ends. Since the central portion of the magnetosome chain is not adjacent to the cell membrane, it must have some other means of support, and a significant fraction of the magnetosomes in these magnetococci cannot be attached to the periplasmic membrane as they apparently are in AMB-1 [28]. In addition to our tomographic preparations of Figs. 3 and 5 , there are hints of this sheath material present - but not commented upon - in several past publications containing TEM images of various magnetotactic bacteria. In particular, Schübbe et al. [48] report a spontaneous mutant of $M$. gryphiswaldense (MRS-1B) that lost an $\sim 80 \mathrm{~kb}$ segment of the magnetosome island and produced no magnetosome vesicles or magnetite crystals. However, as seen on their Fig. 1a, these mutant cells still retain a clear ghost of organic material running along the length of the cells, where the magnetosome chains should have been.

Comparison of the known genetics between the magnetic cocci and magnetospirilla suggests that they probably use similar structures to prevent magnetosome chain collapse. Although uncultured, virtually all magnetic cocci that have been reported so far are members of the alpha-proteobacterial sub-phylum, which also contain the magnetospirilla. The nearly complete genome of the magnetic cocci MC- 1 contains virtually all of the genes in the magnetosome island found in the magnetospirilla, indicating that they share large elements of their magnetosome formation pathways. However, the genomes of the closely related magnetospirilla strains AMB-1 and MS-1 differ in size by nearly $300 \mathrm{~kb}[49,50]$, The apparent association of the magnetosome chain structures with the cytoplasmic membrane in the magnetospirilla most likely results from the cell shape. A $2 \mu \mathrm{m}$ long rod, placed inside a right circular cylinder of about the same length that is then twisted a turn or two (producing the typical structure of a spirillum), will appear to be tangent to the cell wall. Hence a genetic defect that inhibits or minimizes separation of new magnetosome vesicles budding off from the membrane might not be selected against strongly in the magnetospirilla, but would greatly damage the magnetococci. As noted by Vali and Kirschvink [19], endocytosis of this sort was thought to be an exclusively eukaryotic trait.

Although the structure remains uncharacterized, our TEM study of uncollapsed cells reveals the presence of an intracellular magnetosome sheath structure that occupies a significant fraction of the cellular volume. Our failure to image eukaryotic-style internal cytoskel- etal fibers using fixing and staining techniques capable of detecting them in eukaryotes [26] implies that either these proteins are only rarely expressed in strain MS-1 or are not generally assembled in the same fashion as they are in the closely related strains GSR-1 and AMB-1 or in their eukaryotic cousins. As noted earlier, the MS-1 genome is missing $\sim 300 \mathrm{~kb}$ compared to AMB-1, and although the mamJ and mamK genes are present, their expression could be quite different. In this light it is interesting to note that our tomographic results of stained, epon-embedded cells complement and extend the recent studies of Scheffel et al. and Komeili et al. $[27,28]$, who both used cryoTEM on unstained cells of M. gryphiswaldense (with incomplete information from the missing-wedge effect due to the limited tilt-range of $\pm 60^{\circ}$ ) and M. magneticum. Although the classic staining and embedding techniques risk loss of resolution at the sub 5-nm scale, organic structures have such extraordinarily low contrast under any sort of TEM examination that heavy-metal staining is needed to enhance them. It is for this reason that the much larger scale structure of the sheath is apparent in our images.

The recently sequenced genome of M. magnetotacticum contains $4 \mathrm{fts} Z$ homologues of tubulin and two mreB homologues of actin. Of these genes encoding potential structural proteins, one $m r e B$ gene is highly conserved among the genomes of magnetic coccus MC-1, M. magnetotacticum, and M. gryphiswaldense. The highly conserved actin homologue is also part of a larger group (the magnetosome island) of conserved genes containing 18 proteins known to be located on the magnetosome [47,51]. The conserved MreB-like protein, named MamK in M. gryphiswaldense, is not bound to the magnetosome membrane in extracted magnetosomes [47]. The sequence similarity of MamK to MreB and its disconnection from the magnetosome have led to the speculation that it may be involved in the structural control of the magnetosome chain [48]. On the other hand, it is possible that MamK is not involved in the sheath structure and that the sheath is composed of something entirely different, such as a glycoprotein matrix. Future biochemical and genetic work will be able to determine the composition of the sheath.

The nearly identical appearance of magnetosome chains in bacteria to those widespread in eukaryotic cells ranging from protists [52,53] to the magnetoreceptor cells of animals [54-57] has led to the hypothesis that the mechanisms for producing magnetosomes share a common ancestry [58]. Hence the jump from cytoskeletal filaments arranged along the internal cell wall of bacteria to the role of positioning and 
organizing intracellular vacuoles may have been driven by natural selection for magnetotaxis as well as endosymbiotic events in the evolution of the eukaryotic cell. Although there may be no direct homology between the proteins in the magnetosome sheath and the eukaryotic cytoskeleton, the magnetosome structures could provide a stabilizing framework for the evolution of these other scaffolding materials.

The presence of this sheath is also compatible with the observation of Friedmann et al. [13] that the chains of magnetite crystals observed in the magnetite-rich rims of carbonate blebs in the Martian meteorite ALH84001 are, on occasion, encapsulated by an electron-transparent layer of presumed organic composition. If their interpretation of these structures as magnetofossil chains is correct, this transparent material could be the degradation product of the sheath reported here.

\section{Acknowledgments}

This work was partially supported by funds from a NEDO (New Energy and Industrial Technology Development Organization, Japan) Fellowship to A.K., grants NIH 1-RO1-ES-06652, and EPRI-RP2965 to JLK. We thank H. Vali of McGill University for permission to use Fig. 6 a.

\section{Appendix A. Supplementary data}

Supplementary data associated with this article can be found, in the online version, at doi:10.1016/j. eps1.2006.03.041.

\section{References}

[1] Y.A. Gorby, T.J. Beveridge, R.P. Blakemore, Characterization of the bacterial magnetosome membrane, J. Bacteriol. 170 (1988) 834-841.

[2] N. Nakamura, K. Hashimoto, T. Matsunaga, Immunoassay method for the determination of immunoglobulin-G using bacterial magnetic particles, Anal. Chem. 63 (1991) 268-272.

[3] D.A. Bazylinski, R.B. Frankel, Magnetosome formation in prokaryotes, Nat. Rev., Microbiol. 2 (2004) 217-230.

[4] A.J. Kalmijn, R.P. Blakemore, The magnetic behavior of mud bacteria, in: K. Schmidt-Koenig, W.T. Keeton (Eds.), Animal Migration, Navigation and Homing, Springer Verlag, New York, 1978, pp. 354-355.

[5] R.B. Frankel, R.P. Blakemore, Navigational compass in magnetic bacteria, J. Magn. Magn. Mater. 15 (1980) 1562-1564.

[6] R.P. Blakemore, Magnetotactic bacteria, Annu. Rev. Microbiol. 36 (1982) 217-238.

[7] M. Hanzlik, M. Winklhofer, N. Petersen, Spatial arrangement of chains of magnetosomes in magnetotactic bacteria, Earth Planet. Sci. Lett. 145 (1996) 125-134.
[8] R.E. Dunin-Borkowski, M.R. McCartney, R.B. Frankel, D.A. Bazylinski, M. Posfai, P.R. Buseck, Magnetic microstructure of magnetotactic bacteria by electron holography, Science 282 (1998) 1868-1870.

[9] M. Hanzlik, M. Winklhofer, N. Petersen, Pulsed-field-remanence measurements on individual magnetotactic bacteria, J. Magn. Magn. Mater. 248 (2002) 258-267.

[10] J.L. Kirschvink, Paleomagnetic evidence for fossil biogenic magnetite in western crete, Earth Planet. Sci. Lett. 59 (1982) 388-392.

[11] J.L. Kirschvink, On the magnetostatic control of crystal orientation and iron accumulation in magnetosomes, Automedica 14 (1992) 257-269.

[12] V.P. Shcherbakov, M. Winklhofer, M. Hanzlik, N. Petersen, Elastic stability of chains of magnetosomes in magnetotactic bacteria, Eur. Biophys. J. Biophys. Lett. 26 (1997) 319-326.

[13] E.I. Friedmann, J. Wierzchos, C. Ascaso, M. Winklhofer, Chains of magnetite crystals in the meteorite ALH84001: evidence of biological origin, Proc. Natl. Acad. Sci. U. S. A. 98 (2001) 2176-2181

[14] P.R. Buseck, R.E. Dunin-Borkowski, B. Devouard, R.B. Frankel, M.R. McCartney, P.A. Midgley, M. Posfai, M. Weyland, Magnetite morphology and life on Mars, Proc. Natl. Acad. Sci. U. S. A. 98 (2001) 13490-13495.

[15] S.J. Clemett, K.L. Thomas-Keprta, J. Shimmin, M. Morphew, J.R. McIntosh, D.A. Bazylinski, J.L. Kirschvink, S.J. Wentworth, D.S. McKay, H. Vali, E.K. Gibson, C.S. Romanek, Crystal morphology of MV-1 magnetite, Am. Mineral. 87 (2002) 1727-1730.

[16] F.L.H. Brown, Regulation of protein mobility via thermal membrane undulations, Biophys. J. 84 (2003) 842-853.

[17] J.L. Kirschvink, A seventh criterion for the identification of bacterial magnetofossils, EOS, Trans.-Am. Geophys. Union 82 (2001) s131.

[18] A. Witt, K. Fabian, U. Bleil, Three-dimensional micromagnetic calculations for naturally shaped magnetite: octahedra and magnetosomes, Earth Planet. Sci. Lett. 233 (2005) 311-324.

[19] H. Vali, J.L. Kirschvink, Observations of magnetosome organization, surface structure, and iron biomineralization of undescribed magnetic bacteria: evolutionary speculations, in: R. Blakemore (Ed.), Iron Biominerals, Plenum Press, New York, 1991, pp. 97-116.

[20] A.P. Philipse, D. Maas, Magnetic colloids from magnetotactic bacteria: chain formation and colloidal stability, Langmuir 18 (2002) 9977-9984.

[21] D.F. McNeill, J.L. Kirschvink, Early dolomitization of platform carbonates and the preservation of magnetic polarity, J. Geophys. Res., [Solid Earth] 98 (1993) 7977-7986.

[22] S. Cisowski, Interacting vs. non-interacting single-domain behavior in natural and synthetic samples, Phys. Earth Planet. Inter. 26 (1981) 56-62.

[23] A. Kobayashi, T. Taguchi, Biological control of magnetite crystal formation in the magnetotactic bacteria: hints concerning the possible evidence from ALH84001 for life on Mars, Geochim. Cosmochim. Acta 66 (2002) A408.

[24] A. Kobayashi, T. Taguchi, Ultrastructure of the magnetite crystal chains in Magnetospirillum magnetotacticum (MS-1): evidence from TEM tomography for cytoskeletal supporting structures, Geochim. Cosmochim. Acta 67 (2003) A222.

[25] J. Kurner, A.S. Frangakis, W. Baumeister, Cryo-electron tomography reveals the cytoskeletal structure of Spiroplasma melliferum, Science 307 (2005) 436-438. 
[26] O. Medalia, I. Weber, A.S. Frangakis, D. Nicastro, G. Gerisch, W. Baumeister, Macromolecular architecture in eukaryotic cells visualized by cryoelectron tomography, Science 298 (2002) 1209-1213.

[27] A. Scheffel, M. Gruska, D. Faivre, A. Linaroudis, J.M. Plitzko, D. Schuler, An acidic protein aligns magnetosomes along a filamentous structure in magnetotactic bacteria, Nature 440 (2006) 110-114.

[28] A. Komeili, Z. Li, D.K. Newman, G.J. Jensen, Magnetosomes are cell membrane invaginations organized by the actin-like protein MamK, Science 311 (2006) 242-245.

[29] T.T. Moench, W.A. Konetzka, A novel method for the isolation and study of a magnetotactic bacterium, Arch. Microbiol. 119 (1978) 203-212.

[30] G.E. Lee-Whiting, Uniform magnetic fields, in: L. Atomic Energy of Canada, (Ed) report CRT-673, Chalk River Project Research and Development, 1957, p. 8p.

[31] J.L. Kirschvink, Uniform magnetic fields and double-wrapped coil systems: improved techniques for the design of biomagnetic experiments, Bioelectromagnetics 13 (1992) 401-411.

[32] J.L. Kirschvink, Ch. 14. Biogenic ferrimagnetism: a new biomagnetism, in: S. Williamson (Ed.), Biomagnetism: An Interdisciplinary Approach, Plenum Press, New York, NY, 1983, pp. $501-532$.

[33] H.P. Johnson, W. Lowrie, D.V. Kent, Stability of ARM in fine and course grained magnetite and maghemite particles, Geophys. J. R. Astron. Soc. 41 (1975) 1-10.

[34] A.K. Kobayashi, J.L. Kirschvink, M.H. Nesson, Ferromagnets and EMFs, Nature 374 (1995) 123.

[35] A. Komeili, H. Vali, T.J. Beveridge, D.K. Newman, Magnetosome vesicles are present before magnetite formation, and MamA is required for their activation, Proc. Natl. Acad. Sci. U. S. A. 101 (2004) 3839-3844.

[36] K. Dierksen, D. Typke, R. Hegerl, A.J. Koster, W. Baumeister, Towards automatic electron tomography, Ultramicroscopy 40 (1992) 71-87.

[37] J. Frank, T. Wagenknecht, B.F. McEwen, M. Marko, C.E. Hsieh, C.A. Mannella, Three-dimensional imaging of biological complexity, J. Struct. Biol. 138 (2002) 85-91.

[38] J. Frank, Three Dimensional Electron Microscopy of Macromolecular Assemblies, Oxford University Press, Oxford, 2005, $400 \mathrm{pp}$

[39] B. Devouard, M. Posfai, X. Hua, D.A. Bazylinski, R.B. Frankel, P.R. Buseck, Magnetite from magnetotactic bacteria: size distributions and twinning, Am. Mineral. 83 (1998) 1387-1398.

[40] C. Kittel, Introduction to Solid State Physics, John Wiley \& Sons, Inc., New York, 1976, 608 pp.

[41] J.S. Yang, C.R. Chang, Magnetic relaxation in chain-of-spheres ferromagnetic particles, J. Magn. Magn. Mater. 239 (2002) $73-75$.

[42] D.J. Dunlop, O. Ozdemir, Rock Magnetism: Fundamentals and Frontiers, Cambridge University Press, New York, 1997, 573 pp.

[43] M. Schwartz, S.P. Lund, D.E. Hammond, R. Schwartz, K. Wong, Early sediment diagenesis on the Blake/Bahama Outer Ridge, North Atlantic Ocean, and its effects on sediment magnetism, J. Geophys. Res., [Solid Earth] 102 (1997) 7903-7914.
[44] J.L. Kirschvink, H.A. Lowenstam, Mineralization and magnetization of chiton teeth: paleomagnetic, sedimentologic, and biologic implications of organic magnetite, Earth Planet. Sci Lett. 44 (1979) 193-204.

[45] H. Vali, B. Weiss, Y.L. Li, S.K. Sears, S.S. Kim, J.L. Kirschvink, L. Zhang, Formation of tabular single-domain magnetite induced by Geobacter metallireducens GS-15, Proc. Natl. Acad. Sci. U. S. A. 101 (2004) 16121-16126.

[46] D.L. Balkwill, D. Maratea, R.P. Blakemore, Ultrastructure of a magnetotactic spirillum, J. Bacteriol. 141 (1980) 1399-1408.

[47] K. Grünberg, E.C. Muller, A. Otto, R. Reszka, D. Linder, M. Kube, R. Reinhardt, D. Schüler, Biochemical and proteomic analysis of the magnetosome membrane in Magnetospitillum gryphiswaldense, Appl. Environ. Microbiol 70 (2004) 1040-1050.

[48] S. Schübbe, M. Kube, A. Scheffel, C. Wawer, U. Heyen, A. Meyerdierks, H. Madkour, F. Mayer, R. Reinhardt, D. Schüler, Characterization of a spontaneous nonmagnetic mutant of Magnetospirillum gryphiswaldense reveals a large deletion comprising a putative magnetosome island, J. Bacteriol. (2003) 5779-5790.

[49] T. Matsunaga, Y. Okamura, Y. Fukuda, A.T. Wahyudi, Y. Murase, H. Takeyama, Complete genome sequence of the facultative anaerobic magnetotactic bacterium Magnetospirillum sp. strain AMB-1, DNA Res. 12 (2005) 157-166.

[50] L.E. Bertani, J. Weko, K.V. Phillips, R.F. Gray, J.L. Kirschvink, Physical and genetic characterization of the genome of Magnetospirillum magnetotacticum, strain MS-1, Gene 264 (2001) 257-263.

[51] K. Grünberg, C. Wawer, B.M. Tebo, D. Schüler, A large gene cluster encoding several magnetosome proteins is conserved in different species of magnetotactic bacteria, Appl. Environ. Microbiol. 67 (2001) 4573-4582.

[52] D.A. Bazylinski, D.R. Schlezinger, B.H. Howes, R.B. Frankel, S.S. Epstein, Occurrence and distribution of diverse populations of magnetic protists in a chemically stratified coastal salt pond, Chem. Geol. 169 (2000) 319-328.

[53] R.B. Frankel, F.F.T. Dearaujo, M.A. Pires, C.E.M. Bicudo, Magnetotaxis and magnetite in algae, Biophys. J. 49 (1986) A75.

[54] S. Mann, N.H.C. Sparks, M.M. Walker, J.L. Kirschvink, Ultrastructure, morphology and organization of biogenic magnetite from sockeye salmon, Oncorhynchus nerka - implications for magnetoreception, J. Exp. Biol. 140 (1988) 35-49.

[55] C.E. Diebel, R. Proksch, C.R. Green, P. Nielson, M.M. Walker, Magnetite defines a magnetoreceptor, Nature 406 (2000) 299-302.

[56] J.L. Kirschvink, M.M. Walker, C.E. Diebel, Magnetite-based magnetoreception, Curr. Opin. Neurobiol. 11 (2001) 462-467.

[57] M.M. Walker, T.E. Dennis, J.L. Kirschvink, The magnetic sense and its use in long-distance navigation by animals, Curr. Opin. Neurobiol. 12 (2002) 735-744.

[58] J.L. Kirschvink, J.W. Hagadorn, A grand unified theory of biomineralization, in: E. Bäuerlein (Ed.), The Biomineralisation of Nano- and Micro-Structures, Wiley-VCH Verlag $\mathrm{GmbH}$, Weinheim, Germany, 2000, pp. 139-150. 


\title{
Bugbuster-survivability of living bacteria upon shock compression
}

\author{
M.J. Willis ${ }^{\text {a }}$, T.J. Ahrens ${ }^{\text {a,* }}$, L.E. Bertani ${ }^{b}$, C.Z. Nash $^{\text {c }}$ \\ a Lindhurst Laboratory of Experimental Geophysics, Caltech 252-21, Pasadena, CA 91125, United States \\ ${ }^{\mathrm{b}}$ Division of Biological Sci., Caltech 156-29, Pasadena, CA 91125, United States \\ ${ }^{c}$ Division of Geol. Planet. Sci., Caltech 170-25, Pasadena, CA 91125, United States
}

Received 2 April 2005; received in revised form 31 March 2006; accepted 31 March 2006

Available online 16 June 2006

Editor: K. Farley

\begin{abstract}
Shock recovery experiments were conducted on suspensions of $10^{6} / \mathrm{ml}$ E. coli bacteria contained in a water-based medium that is emplaced within stainless steel containers. The water is shocked and recovered. These experiments simulate the environment of bacteria residing either in surface bodies of water or in subsurface water-filled cracks in rocks. Early Earth life is likely to have existed in such environments. However, the E. coli are not believed to be representative of early life and are merely used here for initial experiments. Some $10^{-2}$ to $10^{-4}$ of the bacteria population survived initial (800 ns duration) shock pressures in water of 220 and $260 \mathrm{MPa}$. TEM images of shock recovered bacteria indicate cell wall rupture and delamination. This appears to be the mortality mechanism.

The TEM images indicate cell wall indentations may be occurring as would be consistent with Rayleigh-Taylor or RichtmyerMeshkov fluid instabilities. In the present case, we consider the experiments as representing three layers of fluids: (1) The waterbased medium, a stronger and possibly denser cell wall medium, and the interior of the cell cytoplasm. Variations of only 10-15\% are expected in density. (2) A second mechanism that may cause cell wall failure is the multiple shock (nearly isentropic) compression freezing of liquid water medium into ice VI or ice VII high pressure phase that are $20 \%$ to $25 \%$ denser than the liquid. The decrease in volume associated with the transformation is expected to induce overpressures in the still liquid cell cytoplasm.

Cell dynamic tensile wall strength thus appears to be a critical parameter from either of the above failure modes. Because the strain rate dependence of cell wall tensile strength is unstudied, we utilize the Grady and Lipkin [D.E. Grady, L. Lipkin, Criteria for impulsive rock fracture, Geophys. Res. Lett. 7 (1980) 255-258] model of tensile failure versus time scale (strain rate). Our single datum is fit to this law and we assume that at low strain rates, overpressures exceeding the cell Turgor pressure require on the order of $\sim 10^{3} \mathrm{~s}$. This model which has been applied to brittle media and metals for describing failure may permit application of short duration laboratory experiments as in the present ones to infer responses of organisms to much lower shock pressures, but for longer time scales $\left(10^{0}\right.$ to $\left.10^{3} \mathrm{~s}\right)$ of planetary impacts.

Using the present data for E. coli and applying the Grady and Lipkin model, we find that a $1.5 \mathrm{~km}$ diameter impactor will cause mortality of bacteria within a radius of $10^{2} \mathrm{~km}$ but upon stress related attenuation the subsurface bacteria outside of this radius should survive.
\end{abstract}

(C) 2006 Published by Elsevier B.V.

Keywords: bacteria; shock compression; origin of life; astrobiology

\footnotetext{
* Corresponding author.

E-mail address: tja@caltech.edu (T.J. Ahrens).
} 


\section{Introduction}

Life on Earth has existed since the emergence of clement surface conditions. For the first 500-700 Myr following its formation 4.6 Gyr ago, the Earth was subjected to heavy and continuous impacts from meteoroids which created an environment wholly inhospitable to the survival of primitive organisms, an interval known as the Hadean period. Evidence from lunar cratering indicates that the period of heavy bombardment ended $\sim 3.8 \mathrm{Gyr}$ before the present (e.g. $[2,3])$. Correspondingly, it is generally accepted from fossil evidence that life has existed on Earth for $\sim 3.5$ Gyr (e.g. [4]) and it has been argued that carbon isotopic ratios in rocks from early Archean terranes in Australia and Greenland are evidence of life as far back as $\sim 3.85$ Gyr before the present [5], although such evidence remains controversial $[6,7]$.

Numerous efforts have been made to express the initiation of conditions favorable to the origin and development of life in terms of the frequency and severity of impacts. Maher and Stevenson [8] used the lunar cratering record to define a limiting impact rate below which life was able to develop. An alternative approach by Sleep et al. [6] associated the commencement of life with the time of the most recent planetsterilizing impact; they reasoned that for an impact to be sterilizing it was necessary to vaporize the Earth's oceans. The general framework for evaluating the survival of Mars bacteria upon impact delivery to the Earth was studied by Mileikowsky et al. [9]. Wells et al. [10], reasoning that the Earth's surface would be heated during impacts to an extent that would preclude the survival of organisms, suggested that bacteria may have been able to survive by being ejected into space by impacts and falling back to the Earth after conditions were once again clement. It is generally understood that large impacts pose a threat to life through effects such as the generation of large quantities of dust and aerosols which will reduce the level of sunlight and ignition of large-scale wildfires (e.g. [11]).

Although early giant impacts would be lethal to organisms on the Earth's surface, it is well known that life can survive at large distances below ground. Organisms have been found in rock crevices up to $3 \mathrm{~km}$ underground, obtaining necessary nutrients from rocks (e.g. [12]). These would have been sheltered from the sterilizing effects at the surface during an impact, namely impact heating and physical disruption. However, a shock compression wave will be produced at the point of impact which will travel deep into the Earth. The survivability of organisms to shock compression events may therefore be relevant to understanding the onset and development of life on the early Earth.

In the present bacteria were held in a water-based media, shocked, and the liquid containing bacteria was recovered for analysis. In contrast, in previous studies bacteria were on or in a solid medium. Previous studies are summarized in Table 1.

Burchell et al. [13] impacted bacteria-laden ceramic projectiles into nutrient-rich targets using a two-stage light gas gun, achieving survival at velocities of $5 \mathrm{~km} \mathrm{~s}^{-1}$. The shock pressure quoted in Table 1 is that at the ceramic projectile-agar interface. This does not necessarily have any relation to stress exerted on the bacteria as these are "protected" by the relatively strong grains of ceramic. Burchell et al. [14] have extended this work to the case of bacteria being ejected from icy surfaces during impact, and have also recently determined the bacteria survival rate over a wide range of shock pressures [15]. Mastrapa et al. [16] employed a centrifuge to subject bacteria spores to accelerations of up to $5 \times 10^{6} \mathrm{~ms}^{2}$.

Table 1

Summary of previous experimental studies

\begin{tabular}{|c|c|c|c|c|c|}
\hline Author & Type of experiment & Bacteria used & $\begin{array}{l}\text { Shock pressure } \\
(\mathrm{GPa})\end{array}$ & $\begin{array}{l}\text { Acceleration } \\
\left(\mathrm{m} \mathrm{s}^{-2}\right)\end{array}$ & $\begin{array}{l}\text { Survival } \\
\text { rate }\end{array}$ \\
\hline Burchell et al. [13], [15] & Impact using light gas gun & $\begin{array}{l}\text { Rhodococcus erythropolis/ } \\
\text { Bacillus subtilis }\end{array}$ & $3-78$ (a) & $1.6 \times 109$ & $10^{-4}-10^{-7}$ \\
\hline Mastrapa et al. [16] & Acceleration in centrifuge & Bacillus subtilis/ & $\mathrm{n} / \mathrm{a}$ & $4 \times 106$ & $10^{-1}$ after 65 \\
\hline Mastrapa et al. [16] & Projectile impact & Deinococcus radiodurans & $\mathrm{nd}^{+}$ & $1-5 \times 106$ & $0.4-1.0$ \\
\hline Horneck et al. [17] & $\begin{array}{l}\text { Shock compression } \\
\text { between quartz platens }\end{array}$ & Bacillus subtilis (spores) & 32 & $1.2 \times 109$ & $10^{-4}$ \\
\hline This work & $\begin{array}{l}\text { Recovery of shocked } \\
\text { liquid medium }\end{array}$ & Escherichia coli & 0.26 & $2.6 \times 107$ & $10^{-2}-10^{-4}$ \\
\hline
\end{tabular}

(a) Sample partial dried and deposited on porous alumina.

n/a Not applicable to centrifuge experiment.

(b) Sample placed in cavity in lead bullet.

$\mathrm{nd}^{+}$Pressure/stress on bacteria upon impact of bullet not calculable as contacted geometry between lead bullet and carried bacteria not defined. 


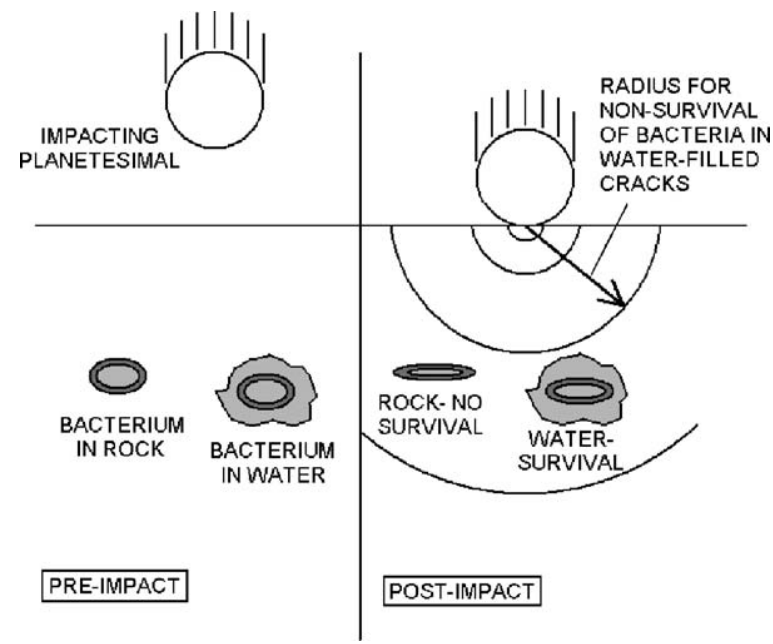

Fig. 1. Comparison between previous experimental studies of survivability of subsurface bacteria during an asteroid impact and current work. Previous studies (Burchell et al. [13,14], Horneck et al. [17]) considered dry bacteria between grains, in rock. In these cases non-hydrostatic strain affects bacteria. In this work bacteria are immersed in water and subjected to only dynamic hydrostatic pressures.

Impact induced deceleration of dried $B$. subtilis spores and $D$. radiodurans bacteria carried in a fluid medium demonstrated nearly complete survival upon exposure to short duration $(5 \mathrm{~ms})$ ballistic tests.

Horneck et al. [17] used a quartz sandwich to dynamically compress dried bacterial spores and was successfully able to culture the spores afterwards. Again, pressures and accelerations apply to the quartz and not bacteria are quoted.

One situation which merits investigation is the degree to which organisms immersed in water in subsurface rock crevices are shielded from the effects of shock induced non-hydrostatic strains. Bacteria within water would be subjected primarily to hydrostatic strain as illustrated in Fig. 1. Recent experiments by Sharma et al. [18] are examining microbial viability in liquid under static hydrostatic compression. In this case, bacteria are able to withstand significant hydrostatic pressures and in some cases survived incorporation into planetary ices.

In this paper we describe an experimental work in which shock recovery experiments were performed on live samples of Escherichia coli (E. coli) bacteria, suspended in a water-based buffer solution. Section 2 describes the sample preparation, experimental method and results. In Section 3, we discuss possible mechanisms that induce cell mortality apparently via cell wall rupture. We suggest a cell wall failure model that may allow extrapolation of the tensile stress associated with failure in short duration laboratory loadings (such as conducted here) to long duration, lower stress conditions associated with planetary impact.

Finally, Section 4 summarizes the main conclusions of this work, namely that the live E. coli bacteria can survive shock pressures of $0.2 \mathrm{GPa}$ for the very short duration $(0.8 \mu \mathrm{s})$ of the present experiments.

\section{Experimental work}

\subsection{The bacteria}

The bacteria sample used in this experiment were $E$. coli. This species was chosen on account of its rapid growth rate and the fact that it is not normally found in laboratory environments, so it should not be confused with any contaminant. However, E. coli should not be considered similar to any wild species that reside within regimes of early microbial life, but is rather a common organism living in the intestines. In constructing scenarios for early life, many have suggested that life developed in anaerobic, reducing environments during planetary accretion, and possibly, at elevated temperatures. The standard E. coli strain MC-4100 F- araD139 $\Delta$ (argF-lac)U169 rpsL1 relA1 deoC1 ptsF25 rboR flb5301 $\lambda^{-}$[15] was used. The structure of a typical $E$. coli cell is shown in Fig. 2. Cells of $E$. coli are lozengeshaped, being $\sim 2 \mu \mathrm{m}$ long and $\sim 0.5 \mu \mathrm{m}$ in diameter. They consist of a central nucleoid, which contains the cell's DNA, surrounded by a cytoplasmic fluid. This is encased in a cytoplasmic membrane and a cell wall. The particular strain of E. coli we used was genetically modified to be resistant to the antibiotic streptomycin. This meant that any recovered samples could be treated with streptomycin to distinguish the original sample from contaminants. Bacteria were cultivated in LB broth, a rich nutrient growth medium composed of

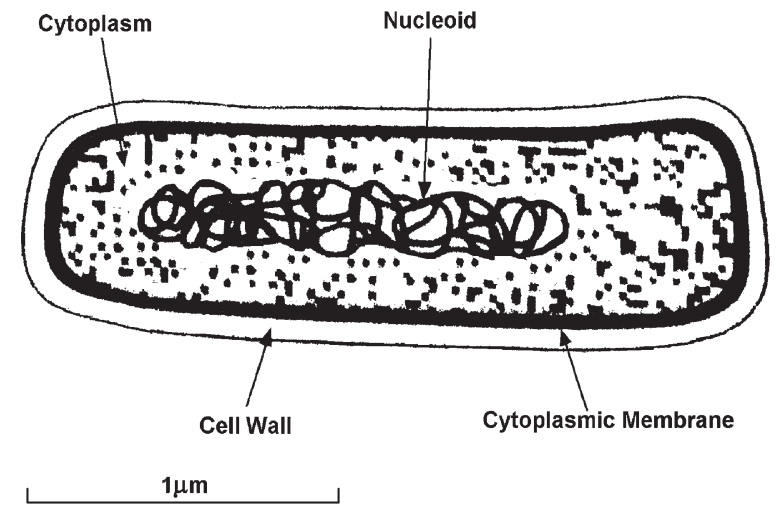

Fig. 2. Structure of E. coli cell. 


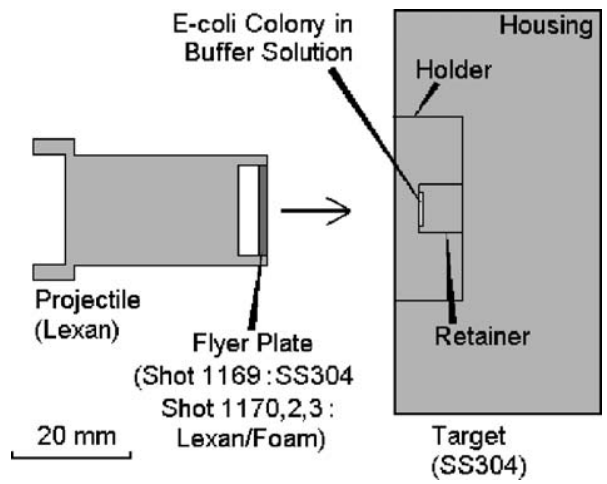

Fig. 3. Illustration of projectile and target. Lexan projectile holds a lexan disk forming flyer plate. A foam disk supports flyer. Stainless steel sample recovery chamber has a $0.5 \mathrm{~mm}$ thick cavity holding bacteria sample in water-based medium.

tryptone and yeast extract. Samples containing $\sim 2 \times 10^{7}$ bacteria were centrifuged to remove the LB broth, and the resultant pellet was suspended in $10 \mathrm{~m}$ MTris, $1 \mathrm{mM}$ EDTA.

\subsection{Experimental procedure}

The recovery chamber used to house the bacteria samples is displayed in Fig. 3. The prepared bacteria samples were loaded into a $10 \mu \mathrm{l}$ cavity in a stainless steel retainer. This was screwed into a stainless steel holder piece with a press fit to seal the liquid sample in place, with an airtight fit provided by a knife-edge around the rim of the cavity in the retainer. This assembly was screwed on to a stainless steel housing component. The chamber design follows that used by Blank et al. [19] for liquid-recovery shock experiments.

The assembled target chamber was mounted on to the end of the barrel of the $20 \mathrm{~mm}$ powder gun in the Lindhurst Laboratory of Experimental Geophysics at Caltech. The gun was used to launch a projectile at the target chamber at velocities of $0.6-1.5 \mathrm{~km} \mathrm{~s}^{-1}$. The projectile, shown in Fig. 3, was composed of lexan and was $80 \mathrm{~mm}$ in length. On the front end of the projectile, a $1.5 \mathrm{~mm}$ thickness disk was mounted, which acted as the flyer plate to drive a planar shock wave through the target assembly containing the bacteria sample. For Shot 1 this flyer plate was composed of stainless steel, but in later shots a lexan flyer plate was used to reduce the shock pressure (see Table 2). For the lexan flyer plates, a $4 \mathrm{~mm}$ thickness disk of polycarbonate foam was mounted behind the flyer. This was inserted to induce a well-defined, nearly rectangular stress versus time shock pulse and to attenuate shock reverberations after the initial shock to reduce the risk of killing bacteria cells which had survived the main shock.

Four shots, plus a control test were carried out using this setup. The experimental conditions for each shot are summarized in Table 2. Initial shock pressures in the bacterial median, assumed to have the properties of liquid water, and the multi-reflected shock pressures assumed to be equal to that of the initial shock pressure in the stainless steel. These were all obtained by the impedance matching method technique [20] using shock and particle velocity data for the various materials involved listed in Table 3. Reflected shock pressures in the samples ranged from 2.2 to $14.9 \mathrm{GPa}$.

Following the shot, the chamber was opened to remove the bacterial sample. In order to separate the sample holder and retainer pieces, it was necessary to drill radially into the holder to loosen the fit. When the retainer had been removed, any liquid bacteria sample remaining in the chamber was extracted using a pipette. It was found that there was not always liquid present after the shot. In this case, $10 \mu 1$ of TE buffer was added to the retainer and was stirred to resuspend the residue. Recovered- or resuspended-samples were mixed with $50 \mu 1$ of LB nutrient broth and spread onto a petri dish containing LB agar medium. The dish was left for $\sim 16 \mathrm{~h}$ at $37^{\circ} \mathrm{C}$ to allow any surviving bacteria to grow.

\subsection{Results and analysis}

The results of the four shots carried out are summarized in Table 2. Because Shot 1 used a stainless steel flyer plate, the shock pressure was considerably greater in this case than in the others. Shot 3 was the only experiment in which liquid sample was recovered from the chamber; in the others, the interior of the

Table 2

Summary of shock recovery experiments

\begin{tabular}{|c|c|c|c|c|c|c|c|}
\hline $\begin{array}{l}\text { Shot } \\
\text { \# }\end{array}$ & $\begin{array}{l}\text { Projectile } \\
\text { type }\end{array}$ & $\begin{array}{l}\text { Impact velocity } \\
\left(\mathrm{km} \mathrm{s}^{-1}\right)\end{array}$ & $\begin{array}{l}\text { Initial shock pressure } \\
\text { in liquid water }(\mathrm{MPa})\end{array}$ & $\begin{array}{l}\text { Peak pressure } \\
(\mathrm{GPa})\end{array}$ & $\begin{array}{l}\text { Liquid sample } \\
\text { recovered? }\end{array}$ & Result & $\begin{array}{l}\text { Survival } \\
\text { rate }\end{array}$ \\
\hline 1 & Steel & 0.74 & 1955 & 14.94 & No & No bacteria observed & 0 \\
\hline 2 & Lexan and foam & 0.76 & 259 & 2.81 & No & Dead bacteria observed & 0 \\
\hline 3 & Lexan and foam & 0.67 & 217 & 2.40 & Yes & Live bacteria recovered & $\sim 10^{-2}$ \\
\hline 4 & Lexan and foam & 0.63 & 199 & 2.23 & No & Live bacteria recovered & $\sim 10^{-4}$ \\
\hline
\end{tabular}




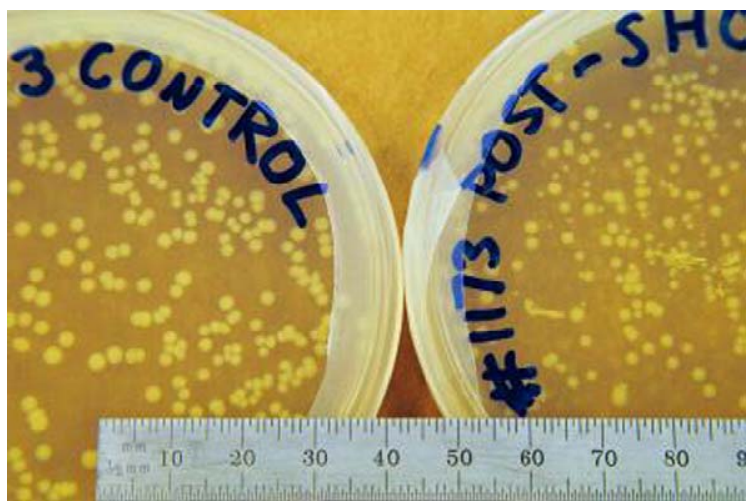

Fig. 4. Control, unshocked (left) and post-shot (right) E. coli bacteria colony plates from Shot 4 . Control plate has been diluted by a factor of $10^{6}$ from the original sample.

sample retainer was found to be dry and so additional TE buffer was used to resuspend the residue.

\subsubsection{Colony counts}

In Shots 1 and 2 no live bacteria were recovered. The petri dish containing recovered sample from Shots 3 and 4, having been mixed with LB broth, spread on agar and left at $37{ }^{\circ} \mathrm{C}$ overnight. On each of the plates, white spots were observed, which are colonies formed by individual surviving bacteria cells. The number of colonies on these plates provides a direct measure of the survival rate for each shot.

For comparison, a dish containing a control sample of the same bacteria (which was not impacted) was cultivated. Each of the control samples were diluted by a factor of $10^{6}$, whereas the shocked samples were not. The control samples for Shots 3 and 4 each contained $\sim 10^{3}$ colonies, consistent with a $1 \mathrm{ml}$ sample of $10^{9}$ bacteria being diluted by a factor of $10^{6}$. The post-shot samples are undiluted from an initial $10 \mu 1$ sample of $10^{7}$ cells, so the number of colonies on these plates is the total number of surviving bacterial from each shot. The post-shot sample from Shot 3 proved to have such a large number of surviving bacteria that in some areas of the plate their colonies merged into a continuum, making an accurate colony count difficult. A value of $8 \times 10^{4}$ determined from the number density of colonies in a region where the colonies are not quite saturated is therefore a lower limit, and the true number of surviving cells is likely $\sim 10^{5}$; the survival rate in the sample is therefore $\sim 10^{-2}$. The post-shot sample from Shot 4, shown in Fig. 4 next to the control sample, contains $\sim 10^{3}$ colonies, leading to a survival rate of $\sim 10^{-4}$. From Fig. 4 it will be observed that an interesting feature of the post-shot colonies from Shot 4 is their heterogeneity in size. Compared to the colonies on the control plate, the shocked colonies vary from being roughly the same size as the unshocked colonies to being considerably smaller. The presence of smaller colonies suggests that these organisms might have sustained some damage during the shock process that had to be repaired before they could begin to multiply and produce colonies.

It will be noted from Table 2 that a significant issue regarding the recovery of live bacteria was the fact that in only one of the four samples was the original liquid recovered, and the other three had to be resuspended. This is a possible factor in the failure to recover any live bacteria in Shots 1 and 2, and the lower survival rate in Shot 4. We presume that liquid was not recovered in three of the shots because of leakage through the fit between the sample retainer and holder. The survival rate in Shot 3 may therefore be representative of that for an impact where bacteria are immersed in water in an enclosed crevice.

\subsubsection{Testing for contamination}

In order to verify that the bacteria colonies observed on the post-shot sample plates in Shots 3 and 4 were cells from the original sample and not due to external contamination, a test shot, $\# 5$, was performed using the TE buffer suspension medium only, without any E.coli being added. In this shot $\# 5,13 \mu 1$ of the medium was sealed into a stainless steel recovery chamber and impacted on by a lexan flyer plate at $0.67 \mathrm{~km} \mathrm{~s}^{-1}$, with a layer of polycarbonate foam behind the flyer plate as in Shots 3 and 4. Following the test shot, dried residue in the chamber was resuspended in TE buffer and spread on a petri dish containing LB agar, being left at $37^{\circ} \mathrm{C}$ for $16 \mathrm{~h}$, as in previous shots, to allow any bacteria colonies to grow.

Table 3a

Analysis of bacteria colonies from contamination test (Shot \#5)

\begin{tabular}{|c|c|c|c|c|c|c|c|}
\hline $\begin{array}{l}\text { Colony } \\
\#\end{array}$ & $\begin{array}{l}\text { Colony diam. } \\
(\mathrm{mm})\end{array}$ & $\begin{array}{l}\text { Colony } \\
\text { color }\end{array}$ & $\begin{array}{l}\text { Cell } \\
\text { shape }\end{array}$ & $\begin{array}{l}\text { Cell diam. } \\
(\mu \mathrm{m})\end{array}$ & $\begin{array}{l}\text { Lambda coliphage } \\
\text { sensitivity }\end{array}$ & $\begin{array}{l}\text { Streptomycin } \\
\text { resistant? }\end{array}$ & $\begin{array}{l}\text { Possible } \\
\text { identification }\end{array}$ \\
\hline 1 & 6 & Off-white & Rod & $10-20$ & No & No & Bacillus \\
\hline $2-4,6$ & 2 & White & Round & 1 & No & No & Micrococcus \\
\hline $5,7,8$ & 2 & Yellow & Round & 1 & No & No & Staphylococcus \\
\hline
\end{tabular}


Table $3 b$

Analysis of bacteria colonies from contamination test (Shot \#5)

\begin{tabular}{llllllll}
\hline $\begin{array}{l}\text { Colony } \\
\#\end{array}$ & $\begin{array}{l}\text { Colony diam. } \\
(\mathrm{mm})\end{array}$ & $\begin{array}{l}\text { Colony } \\
\text { color }\end{array}$ & $\begin{array}{l}\text { Cell } \\
\text { shape }\end{array}$ & $\begin{array}{l}\text { Cell diam. } \\
(\mu \mathrm{m})\end{array}$ & $\begin{array}{l}\text { Lambda coliphage } \\
\text { sensitivity }\end{array}$ & $\begin{array}{l}\text { Streptomycin } \\
\text { resistant? }\end{array}$ & $\begin{array}{l}\text { Possible } \\
\text { identification }\end{array}$ \\
\hline E. coli & 1 & White & Rod & 1 & Yes & Yes & E. coli \\
\hline
\end{tabular}

Having been left overnight, 8 bacteria colonies were found to have grown on the petri dish. Various tests were performed to determine whether any of these were $E$. coli; the results of this analysis are summarized in Table 3a. First, the appearance of the colonies themselves was compared with the expected characteristics of colonies produced by E. coli. From Table 3b it will be observed that while E. coli colonies are white and $2 \mathrm{~mm}$ in diameter after $16 \mathrm{~h}$, colony 1 is off-white and $6 \mathrm{~mm}$ in diameter, and colonies 5, 7 and 8 are yellow. From the colony appearance only $2-4$ and 6 remain as E. coli candidates.

The second test was observation of cellular morphology. The colonies consist of a large number of cells, so to observe the cells in a given colony it was necessary to take a portion of the colony, suspend it in TE buffer and place a drop of the resultant solution onto a microscope slide. The sample was then studied using an optical microscope at $400 \times$ magnification. As will be seen in Fig. 2, E. coli cells are rod-shaped and $\sim 1 \mu \mathrm{m}$ in diameter. It will be observed that the cells from colony 1 , while being rod-shaped, are much larger $(10-20 \mu \mathrm{m})$ than E. coli cells, and cells from colonies 2 to 8 , although the correct size, are round in shape. Therefore none of the observed colonies have cell morphologies consistent with $E$. coli.

Another test was to determine their bacteriophage sensitivity. Bacteria are the unwilling hosts to a variety of bacteriophages, or bacterial viruses. Different types of bacteria are sensitive to (i.e. can be killed by) specific bacteriophages: $E$. coli is sensitive to coliphages, but no other types of bacteria are. Hence, this bacteriophage can be used to test whether a given colony consists of $E$. coli. All of the contaminants were tested for sensitivity to a typical coliphage called "lambda". As will be noted from Table 3, none were found to be sensitive, indicating that none are E. coli.

The final test performed on the colonies from the test shot was resistance to streptomycin. The strain of $E$. coli used in Shots 1-4 was given a resistance to the antibiotic streptomycin. It was found that when streptomycin was added to each of the contaminant colonies, the colonies were killed. As none of the colonies in the test shot have resistance to streptomycin, none are consistent with the bacteria samples from Shots 1 to 4 .
We conclude from the various tests performed that none of the observed colonies are produced by E. coli, and thus the $E$. coli colonies recovered from shots 3 to 4 do not originate from external contamination.

\subsubsection{Optical microscopy with stain}

Having extracted a sample to test for the presence of live bacteria, the interior of the sample retainer was coated with a fluorescent staining dye (DAPI: 4',6Diamidino-2-phenylindole) and examined under an optical microscope. The dye binds to DNA and therefore allows organisms to show up by their strong fluorescence. As the dye is lethal to the bacteria so it was merely used to determine the physical presence of bacteria in the recovered sample. The process by which the stain allows cell identification is illustrated in Fig. 5.

Fig. 6 displays a series of epifluorescence microscope images of DAPI-stained bacteria in the sample retainer after shooting, with ultraviolet light being shone on the sample. Images of Shot 2 are shown in Fig. 6(a) and (b) and Shot 4 in Fig. 6(c) and (d). For comparison, an image of a DAPI-stained unshocked $E$. coli sample on a microscope slide is shown in Fig. 6(e). In each of the images, individual $E$. coli cells are visible as bright dots. In Fig. 6(a) cells are present in the upper right of the image, with a heavy concentration visible as a bright streak. In Fig. 6(b) a small number of cells appear in the upper right, with an additional concentration towards

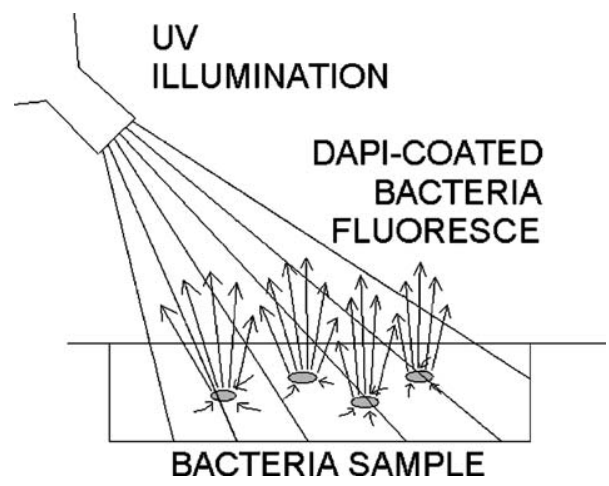

Fig. 5. Illustration of how DAPI staining highlights position of bacteria cells. E. coli are soaked in (lethal) DAPI fluorescent stain. Stain binds to DNA and thus indicates where cells are concentrated upon UV light illumination. 

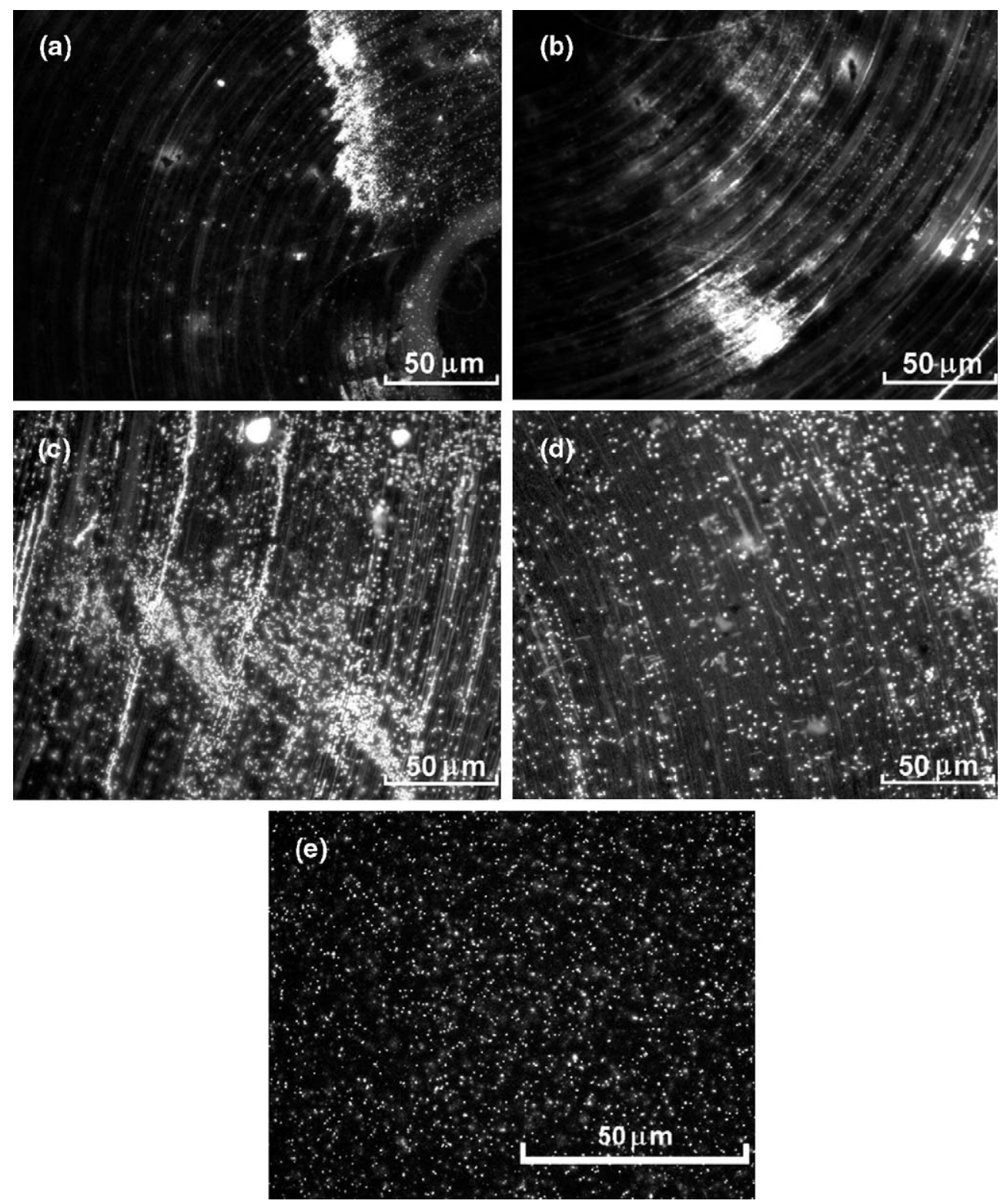

Fig. 6. Images of interior of sample recovery chambers, using DAPI fluorescent stain to identify position of bacteria cells. (a) and (b) are from Shot 2 , and (c) and (d) are from Shot 4. Bacteria cells show up as tiny bright dots, sometimes concentrated in localized regions. For comparison, more uniform distribution of $E$. coli unshocked is shown in (e).

the bottom of the picture. Shot 4, Fig. 6(c) and (d), display bacteria cells all over the images, with (c) having a higher concentration towards the lower part of the image. In the comparison picture of unshocked bacteria in Fig. 6(e), cells appear as uniformly distributed bright dots.

From Fig. 6 it will be seen that bacteria cells are clearly visible in the recovery chambers from Shots 2 to 4. It appears from Fig. 6(a) and (b) that only a small quantity of the original bacteria sample was still in the chamber after Shot 2, while after Shot 4 most of the original sample was still visible. These qualitative observations of post-shock bacteria population are consistent with the results of Table 2 and Fig. 4.

\subsubsection{TEM analysis}

A further analysis technique to examine the physical damage to $E$. coli cells from shock compression employed the use of transmission electron microscopy 

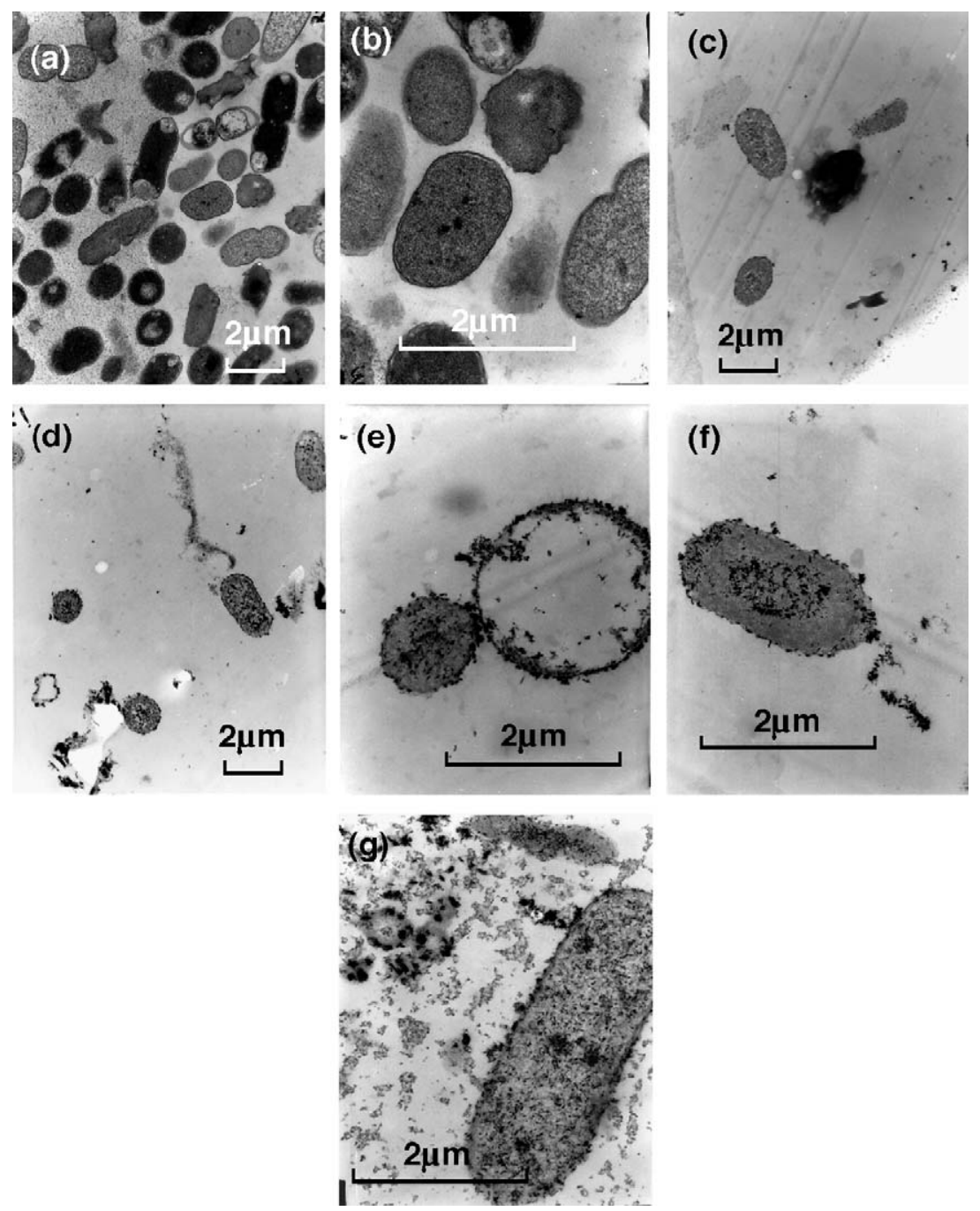

Fig. 7. Transmission electron microscope (TEM) images of control sample of $E$. coli bacteria, at (a) $25,000 \times$ and (b) $75,000 \times$ magnification; and postshot samples from Shot 4 at (c), (d) 25,000× and (e) to (g) 75,000× magnification.

(TEM). Using the Phillips 201 TEM facility (at operating voltage of $60 \mathrm{kV}$ ) in Caltech's Division of Biological Sciences we analyzed control and post-shot samples of $E$. coli cells from Shot 4 . Each sample was prepared by replacing the liquid in the sample with progressively more viscous polymers until a solid was produced. After this, a $30 \mathrm{~nm}$ section was cut from the sample and a beam of electrons was used for imaging. The resultant images from this process are shown in Fig. 7. Fig. 7(a) and (b) are TEM images taken of the control sample, at a magnification of $25,000 \times$ and $75,000 \times$ respectively. In each image individual cells of $E$. coli show up as ovals or circles, and their internal structure is clearly visible. Fig. 7(c) and (d) are TEM images of the post-shot sample at a magnification of $25,000 \times$, and Fig. 7(e) to (g) show post-shot TEM images at $75,000 \times$ magnification. Comparison of each of the post-shot images with the control sample images illustrates that very few cells are still visible. Furthermore, the surviving cells show clear signs of damage: there is evidence of cell wall rupture and a delineation.

\section{Possible shock-induced mortality mechanisms and planetary scaling}

In this section we first note the nature of damage to cells, formulate two possible cell mortality mechanisms, and on this basis suggest a scaling model. The TEM images, Fig. 7(e), (f), and (g), show broken cell walls and partial defoliation of cell wall material from the main cell mass. 
We suggest on the basis of these images that:

(1) Either local overpressurization followed by tensile failure of cell walls occurred. One possible mechanism for cell wall overpressurization is to assume that the water media surrounding the cell has transformed to a denser ice VI or ice VII with the density increase of $20-25 \%$, but the water in the cell cytoplasm has not. This transformation in water has been observed by Dolan and Gupta [21]. This transformation is also observed by Sharma et al. [18] in a static study of compression of E. coli.

(2) The undulation of cell walls suggests that these may be remnants of Richtmyer-Meshkov instabilities induced by the marked shock acceleration of the material when encompassed by the shock or in the case of slightly different media, we can consider three different layers: the higher impedance cell walls and the lower impedance cytoplasm - in the interior of the cells, as well as the lower impedance media outside the cells. In the general case where the accelerations may not necessarily be occurring as a result of being encompassed by the shock, these types of instabilities are called Rayleigh-Taylor. Both contrasts in density and viscosity appear to give rise to the growth of these types of instabilities. Contrasts in viscosity can be related to differences in yield strength. At a given strain rate, these may be related to the stresses discussed inducing plastic deformation as discussed by Colvin et al. [22]. Since tensile strength can be considered a controlling parameter in both mechanisms, we assume the Grady and Lipkin [1] tensile behavior is given by:

$\sigma_{\mathrm{cw}}=a / t_{\mathrm{d}}^{1 / b}$

where $a$ and $b$ are positive constants and $t_{\mathrm{d}}$ is stress duration. Thus an equation of the form of Eq. (1) implies that the dynamic tensile strength of the cell wall $\left(\sigma_{\mathrm{cw}}\right)$ can be substantially higher when a short duration stress is applied.

The present experiments provide a critical shock pressure in water that we assume, equals the cell wall tensile strength, at a very short time duration. Thus, to constrain the $a$ and $b$ parameters, we assume the onset of mass mortality for very high stresses and very short (800 ns) durations occurs in our experiment for Shot 3, at $218 \mathrm{MPa}$. Obviously, another plausible constraint is required. We also assume that at very long times there is a minimal stress that will just induce cell wall tensile failure. To quantify the long time scale failure condition, we assume this occurs over a very long time scale that is comparable to those occurring in planetary impacts. For example, an impactor with a very large diameter such as $\sim 10^{3} \mathrm{~km}$ induces such long duration stresses. The time scale of the stress pulse will then be $\sim 10^{3} \mathrm{~s}$ in the case of, for example, a porous impactor which has a very low shock velocity. We assume that the tensile strength of the cell wall approaches the Turgor (cell interior) pressure at these long time scales. Turgor pressures in bacteria, such as E. coli are measured at $0.15 \mathrm{MPa}$ [23]. These also depend in detail on salt content of the cell cytoplasm. Assuming the above two tensile stresses and times in Eq. (1), we obtain $a=1.26$ and $b=2.72$, where $t_{\mathrm{d}}$ is in seconds and $\sigma_{\mathrm{cw}}$ is in MPa.

We note that the value of $b$ of 2.72 , is comparable to, but slightly lower than, the values of 3-4 measured for non-biogenic materials $[1,24]$.

The tensile stress in the cell wall, in this case, is considered as a membrane surrounding a pressurized fluid (cell cytoplasm). Thus we assume that the effective tensile strength of the cell wall varies with time duration of the transient stress pulse, and hence effectively the size of this impactor. The time duration of the stress pulse is approximated by

$t_{\mathrm{d}} \cong 2 d / U_{\mathrm{s}}^{\mathrm{p}}$

where $d$ is the impactor diameter and $U_{\mathrm{s}}^{\mathrm{p}}$ is the shock velocity in the impactor calculated from the impedance match solution of the projectile target shock interaction [25].

\section{Application to shock pressure decay for impacts on the earth}

As a planetary impact is sketched in Fig. 1, we require knowledge of the survivability of bacteria within cracks in the Earth upon impact. We assume that surviving bacteria are located beyond the radius where the dynamic pressure exceeds the cell wall tensile stress using the same logic as previously discussed for life survival by Cabrol et al. [26], where a very similar model also based on Ahrens and O'Keefe [27] scaling. For a radius, $r$, around the impact site, comparable to the impactor radius, $r_{1}$, the shock pressure is given by the impedance match solution. For Earth impact, where the impact velocities of projectiles with $r_{1} \geq 10 \mathrm{~m}$ are 
Table 4

Shock and particle velocity data

\begin{tabular}{lllll}
\hline Material & $\rho_{0}$ & $C_{0}$ & $S$ & Source \\
\hline $\begin{array}{l}\text { Stainless } \\
\quad \text { steel 304 }\end{array}$ & 7.87 & 4.58 & 1.49 & Duffy [28] \\
Lexan & 1.191 & 2.427 & 1.498 & $\begin{array}{l}\text { Marsh [29] } \\
\text { Water }\end{array}$ \\
& 0.998 & 1.502 & 1.966 & $\begin{array}{l}\text { Marsh [29], } \\
\text { Rybakov and } \\
\end{array}$ \\
& & & & $\begin{array}{l}\text { Rybakov [30] } \\
\text { Gabbro }\end{array}$ \\
\hline
\end{tabular}

greater than Earth escape velocity $\left(11.2 \mathrm{~km} \mathrm{~s}^{-1}\right)$, the shock pressures in the impedance match region are sufficiently high that they always exceed the cell wall strength. Outside $r_{1}$, the shock pressure, $P$, decays and is approximated by

$P=P_{0}\left(r / r_{1}\right)^{n}$

where

$n \cong-0.625 \log _{10}\left[V_{i}(\mathrm{~km} / \mathrm{s})\right]-0.125$

Upon shock decay when $P \leq \sigma_{\mathrm{cw}}$, the bacterium survives. As an example, we calculate survival radii as in Table 5 for several impact velocities and assumed impactor properties: a gabbro impactor at 11 and $20 \mathrm{~km}$ $\mathrm{s}^{-1}$, and an ice impactor at $30 \mathrm{~km} \mathrm{~s}^{-1}$, with impactor diameters between $1.5 \mathrm{~mm}$ (our experimental condition) and $50 \mathrm{~km}$. These were calculated using the shock wave parameters of Table 4.

Table 5 demonstrates that a $1.5 \mathrm{~km}$ diameter projectile induces a shock wave with a hundred $\mathrm{km}$ kill radius. Upon increasing the impactor radius to giant impact, we find that in the $50 \mathrm{~km}$ diameter range, the kill radius becomes close to the Earth diameter $(12,800 \times$ $10^{3} \mathrm{~m}$ ) implying that no bacteria is sustainable for impacts larger than this value. Here we are simply suggesting order of magnitude of the dimensions of the kill zone. Obviously, we are not taking into account wave reflection for a free-surface that also controls dynamic stresses. Predictive results such as shown in Table 5 may allow investigation of the effects of a range of impactor diameters such projectiles could be expected in an actual planetary impact history.

\section{Conclusions}

Impact experiments conducted on colonies of $10^{7} \mathrm{E}$. coli bacteria concentrated in $10 \mathrm{~mm}^{3}$ water-based growth media contained within stainless steel were conducted. Initial shock pressures of $200-300 \mathrm{MPa}$ resulted in mortality of organisms. Some $10^{-2}$ and $10^{-4}$ survived 218 and $258 \mathrm{MPa}$, respectively, for durations of 800 ns. TEM images suggest that mortality was induced by overpressurizing cell cytoplasm, possibly as a result of transformation of water medium surrounding the cell to ice VI or ice VII. Another hypothesis is that mortality was induced by strong acceleration of material by the shock front and differentially affecting the varying densities of the media in the cell. Here Richtmyer-Meshkov instabilities deformed cell walls. The degree that leakage of impacted water media from the samples could affect the statistics of bacterial survival has not been studied, but clearly this may be another factor. We believe our experimental arrangement has similarities to the sighting of early life organisms living in water-filled cracks in rock. We assume for short time scale pulses (800 ns) the critical shock pressures (200-300 MPa) in water reflect overpressures and these equal dynamic tensile strengths of cell wall media. We assume that tensile failure strength $\sigma_{\mathrm{cw}}$ depends on time scale of the impact event, $t_{\mathrm{d}}$ according to the Grady-Lipkin (Eq. 1) relations previously observed in non-biogenic media. Where $a$ and $b$ are constants evaluated from $\sigma_{1 \mathrm{cw}}=218 \mathrm{MPa}$ and $t=800 \mathrm{~ns}$ (laboratory conditions) and $\sigma_{2 \mathrm{cw}}=150 \mathrm{kPa}$ and $t_{2}=10^{3} \mathrm{~s}$ corresponding to Turgor pressure of $E$. coli and the radii $\left(\sim 10^{3} \mathrm{~km}\right)$ of the largest Earth impactor object, yielding time scales of loading of $\sim 10^{3} \mathrm{~s}$. These values should be considered as very speculative examples.

Future experimentation may allow determining $a$ and $b$ parameters for different organisms and shock (duration) loading systems. Using the bounds given above yield: $a=1.26$ and $b=2.72$ where $\sigma_{\mathrm{cw}}$ is in $\mathrm{MPa}$ and $t_{\mathrm{d}}$ in

Table 5

Predicted kill radius for various impactors

\begin{tabular}{|c|c|c|c|c|c|c|}
\hline $\begin{array}{l}\text { Impactor } \\
\text { material }\end{array}$ & $\begin{array}{l}\text { Impact velocity } \\
\left(\mathrm{km} \mathrm{s}^{-1}\right)\end{array}$ & $\begin{array}{l}\text { Peak pressure at } \\
\text { point of impact } \\
(\mathrm{GPa})\end{array}$ & $\begin{array}{l}\text { Kill radius for } \\
\text { impactor } D=1.5 \mathrm{~mm} \\
\text { (m) }\end{array}$ & $\begin{array}{l}\text { Kill radius for } \\
\text { impactor } D=1.5 \mathrm{~km} \\
\text { (m) }\end{array}$ & $\begin{array}{l}\text { Kill radius for } \\
\text { impactor } D=15 \mathrm{~km} \\
\text { (m) }\end{array}$ & $\begin{array}{l}\text { Kill radius for } \\
\text { impactor } D=50 \mathrm{~km} \\
\text { (m) }\end{array}$ \\
\hline Gabbro & 11 & 189 & 0.0096 & $127 \times 103$ & $1980 \times 10^{3}$ & $8350 \times 10^{3}$ \\
\hline Gabbro & 20 & 506 & 0.0120 & $129 \times 103$ & $1940 \times 10^{3}$ & $7980 \times 10^{3}$ \\
\hline Ice & 30 & 540 & 0.0099 & $93.8 \times 103$ & $1390 \times 10^{3}$ & $5660 \times 10^{3}$ \\
\hline
\end{tabular}


seconds. The rate effect on tensile strength observed here is slightly greater than the $3<b<4$ values that are obtained previously $[1,24]$ for various non-biogenic media.

If the above models are valid, using the shock pressure decay model of Ahrens and O'Keefe [27] for Earth impactors suggests that for $1.5 \mathrm{~km}$ diameter objects induce kill radii of 94-129 km whereas giant, $50 \mathrm{~km}$ diameter impactors induced kill radii close to Earth's diameter, and hence approach total subsurface microbial mortality.

\section{Acknowledgments}

This research is supported by the National Aeronautics and Space Administration under Grant No. NNG04GI07G issued through NASA/Goddard Space Flight Center. We thank Editor Kenneth Farley for his encouragement and patience, reviewer, Rachel Maestra, an anonymous reviewer, for a series of helpful comments and suggestions, and Professor Carrine Blank. Professors Joseph Kirschvink and Dianne Newman also proffered comments and advice. Division of Geological and Planetary Sciences contribution \#9089.

\section{References}

[1] D.E. Grady, L. Lipkin, Criteria for impulsive rock fracture, Geophys. Res. Lett. 7 (1980) 255-258.

[2] W.K. Hartmann, G. Ryder, K. Dones, D. Grinspoon, The timedependent intense bombardment of the primordial Earth/Moon system, in: R.M. Canup, K. Righter (Eds.), Origin of the Earth and Moon, University of Arizona Press, Tucson, 2000, pp. 493-512.

[3] D. Stöffler, G. Ryder, Stratigraphy and isotope ages of lunar geologic units: chronological standard for the inner solar system, Space Sci. Rev. 96 (2001) 9-54.

[4] E.G. Nisbet, The Beginnings of Life, Chapt. 4, The Young Earth: An Introduction to Archaen Geology, Allen and Unwin, Boston, 1987.

[5] S.J. Mojzsis, G. Arrhenius, K.D. McKeegan, T.M. Harrison, A.P. Nutman, C.R.L. Friend, Evidence for life on Earth before 3,800 million years ago, Nature 384 (1996) 55-59.

[6] N.H. Sleep, K.J. Zahnle, K.F. Kasting, H.J. Morowitz, Annihilation of ecosystems by large asteroid impacts on the early Earth, Nature 342 (1989) 139-142.

[7] M.D. Brasier, O.R. Green, A.P. Jephcoat, A.K. Kleppe, M.J. Van Kranendonk, J.F. Lindsay, A. Steel, N.V. Grassieau, Questioning the evidence for Earth's oldest fossils, Nature 416 (2002) 76-81.

[8] K.A. Maher, D.J. Stevenson, Impact frustration of the origin of life, Nature 331 (1988) 612-614.

[9] C. Mileikowsky, F.A. Cucinotta, J.W. Wilson, B. Gladman, G. Horneck, L. Lindegren, J. Melosh, H. Rickman, M. Valtonen, J.Q. Zheng, Natural transfer of viable microbes in space: 1. from Mars to Earth and Earth to Mars, Icarus 145 (2000) 391-427.
[10] L.E. Wells, J.C. Armstrong, G. Gonzalez, Reseeding of early Earth by impacts of returning ejecta during the late heavy bombardment, Icarus 162 (2003) 38-46.

[11] R.A.F. Grieve, Extraterrestrial impacts on Earth: the evidence and the consequences, in: M.M. Grady, R. Hutchison, G.J.H. McCall, D.A. Rotherey (Eds.), Meteorites: Flux with Time and Impact Effects. Special Publication, vol. 140Geological Society of London, London, 1998, pp. 105-131.

[12] M.R. Fisk, M.C. Storrie-Lombardi, S. Douglas, R. Popa, G. McDonald, C. Di Meo-Savoie, Evidence of biological activity in Hawaiian subsurface basalts, Geochem. Geophys. Geosyst. $\left(\mathrm{G}^{3}\right)$ 4 (2003) 1103, doi:10.1029/2002GC000387.

[13] M.J. Burchell, J. Mann, A.W. Bunch, P.F.B. Brandão, Survivability of bacteria in hypervelocity impact, Icarus 154 (2001) $545-547$.

[14] M.J. Burchell, J.A. Galloway, A.W. Bunch, P.F.B. Brandão, Survivability of bacteria ejected from icy surfaces after hypervelocity impact, Orig. Life Evol. Biosph. 33 (2003) 53-74.

[15] M.J. Casadaban, Transposition and fusion of LAC genes to selected promoters in Escherichia coli using BacteriophaseLambda and Bacteriophase-Mu, J. Mol. Biol. 104 (1976) $541-555$.

[16] R.M.E. Mastrapa, H. Glanzberg, J.N. Head, H.J. Melosh, W.L. Nicholson, Survival of bacteria exposed to extreme acceleration: implications for panspermia, Earth Planet. Sci. Lett. 189 (2001) $1-8$.

[17] G. Horneck, D. Stöffler, U. Eschweiler, U. Hornemann, Bacterial spores survive simulated meteorite impact, Icarus 149 (2001) 285-290.

[18] A. Sharma, J.H. Scott, G.D. Cody, M.L. Fogel, R.M. Hazen, R.J. Hemley, W.T. Huntress, Microbial activity at gigapascal pressures, Science 295 (2002) 1514-1516.

[19] J.G. Blank, G.H. Miller, M.J. Ahrens, R.E. Winans, Experimental shock chemistry of aqueous amino acid solutions and the cometary delivery of prebiotic compounds, Origins of Life and Evolution of the Biosphere, vol. 31, Kluwer Acad. Publ., The Netherlands, 2001, pp. 15-51.

[20] Y.B. Zel'dovich, Y.P. Raizer, Physics of Shock Waves and HighTemperature Hydrodynamic Phenomena, Academic, New York, 1967, 916 pp.

[21] D.H. Dolan, Y.M. Gupta, Time-dependent freezing of water under dynamic compression, Chem. Phys. Lett. 374 (2003) 608-612.

[22] J.D. Colvin, M. Legrand, B.A. Remington, G. Schurtz, S.V. Weber, A model for instability growth in accelerated solid metals, J. Appl. Phys. 93 (2003) 5287-5301.

[23] D.S. Cayley, H.J. Guttman, M.T. Record Jr., Biophysical characterization of changes in amounts and activity of Escherichia coli cell and compartment water and turgor pressure in response to osmotic stress, Biophys. J. 78 (2000) $1748-1764$.

[24] H.-A. Ai, T.J. Ahrens, Dynamic tensile strength of terrestrial rocks and application to impact cratering, Meteorit. Planet. Sci. 39 (2004) 233-246.

[25] T.J. Ahrens, Shock wave techniques for geophysics and planetary physics, in: C.G. Sammis, T.L. Henyey (Eds.), Methods of Experimental Physics 24, Part A, Academic Press, New York, 1987, pp. 185-235.

[26] N.A. Cabrol, D.D. Wynn-Williams, D.A. Crawford, E.A. Grin, Recent aqueous environments in Martian impact craters: an astrobiological perspective, Icarus 154 (2001) 98-112. 
[27] T.J. Ahrens, J.D. O'Keefe, Impact on the Earth, ocean, and atmosphere, Int. J. Impact Eng. 5 (1987) 13-32.

[28] T.S. Duffy, Elastic properties of metals and minerals under shock compression, Ph.D., California Institute of Technology, Pasadena, CA, 1992.
[29] S.P. Marsh (Ed.), LASL Shock Hugoniot Data, University of California Press, Berkeley, 1980, 658 pp.

[30] A.P. Rybakov, I.A. Rybakov, Polymorphism of shocked water, Eur. J. Mech. B, Fluids 14 (1995) 323-332. 